

\title{
Le problème de Bogomolov effectif sur les variétés abéliennes
}

\author{
Aurélien Galateau
}

\begin{abstract}
On obtient une nouvelle minoration du minimum essentiel en petite codimension sur les variétés abéliennes, sous une conjecture concernant leurs idéaux premiers ordinaires. Cette minoration, déjà connue dans le cas torique depuis les travaux d'Amoroso et David, est optimale «à $\varepsilon$ près » en le degré de la sous-variété. La preuve suit la méthode des pentes et est basée sur les propriétés $p$-adiques des points de torsion des variétés abéliennes.

We give a new lower bound for the essential minimum of subvarieties of abelian varieties with small codimension, under a conjecture about ordinary primes in abelian varieties. This lower bound, known in the toric case through the work of Amoroso and David, is best "up to an $\varepsilon$ " in the degree of the subvariety. The proof follows the slope method and is based on the $p$-adic properties of torsion points in abelian varieties.
\end{abstract}

\section{Introduction}

L'objet de ce travail est de minorer le minimum essentiel sur les variétés abéliennes. Une telle minoration est une version quantitative de la conjecture de Bogomolov. Soit $C$ une courbe algébrique de genre $g \geq 2$ définie sur $\overline{\mathbb{Q}}$ et plongée dans sa jacobienne $J(C)$. On note $L$ le fibré canonique et $\hat{h}_{L}$ la hauteur de Néron-Tate associée. Le théorème suivant a été conjecturé par Bogomolov.

Théorème 1.1 (Ullmo). Il existe $\varepsilon>0$ tel que $\left\{x \in C(\overline{\mathbb{Q}}): \hat{h}_{L}(x) \leq \varepsilon\right\}$ est fini.

Plus généralement, soit $V$ une sous-variété algébrique d'une variété abélienne $A$ munie d'un fibré $L$ ample et symétrique, et $\hat{h}_{L}$ la hauteur de Néron-Tate associée à ce fibré (ici et dans toute la suite de cet article, une variété est toujours supposée irréductible et définie sur $\overline{\mathbb{Q}})$. On commence par donner un analogue en dimension supérieure de l'hypothèse faite précédemment sur le genre.

MSC2000: primary 11G10; secondary 11J81, 14G40.

Mots-clefs: Bogomolov, variété abélienne, minoration, hauteur, abelian variety, lower bound, height. 
Définition 1.2. On dit que $V$ est de torsion si $V$ est la translatée d'une sous-variété abélienne par un point de torsion.

On introduit aussi le minimum essentiel, qui renseigne sur les points de petite hauteur dans $V$.

Définition 1.3. Le minimum essentiel de $V$ est :

$$
\hat{\mu}_{L}^{\mathrm{ess}}(V)=\inf \left\{\theta>0: \overline{V(\theta)}^{Z}=V(\overline{\mathbb{Q}})\right\},
$$

où $V(\theta)=\left\{x \in V(\overline{\mathbb{Q}}), \hat{h}_{L}(x) \leq \theta\right\}$ et $\overline{V(\theta)}{ }^{Z}$ est son adhérence de Zariski.

On peut maintenant donner une généralisation de la conjecture de Bogomolov en dimension supérieure.

Théorème 1.4 (Zhang). Soit $V$ une sous-variété d'une variété abélienne A. Le minimum essentiel de $V$ est nul si et seulement si $V$ est de torsion.

Le résultat analogue est vrai si on remplace $A$ par un tore [Zhang 1992] ou plus généralement par une variété semi-abélienne [David et Philippon 2000].

On peut donner une version quantitative de ce résultat. Ceci revient, en dimension générale, à minorer le minimum essentiel d'une variété qui n'est pas de torsion. Par le théorème des minima successifs démontré par Zhang [1995a], il est équivalent de minorer la hauteur d'une telle variété. Depuis les travaux de Bombieri et Zannier (voir [1995] pour le cas torique et [1996] pour le cas abélien), on sait qu'on peut espérer obtenir une borne « uniforme » pour le minimum essentiel, ne dépendant que du degré de $V$ et de la variété abélienne $A$.

Dans le cas torique, Amoroso et David [2003] donnent une minoration optimale aux facteurs logarithmiques près en le degré de $V$. Le degré y est remplacé par un invariant plus fin qui apparaît naturellement avec les techniques diophantiennes, l'indice d'obstruction.

Définition 1.5. Soit $V$ une sous-variété stricte de $S$ une variété semi-abélienne munie de $L$ un fibré ample. On définit l'indice d'obstruction de $V$, noté $\omega_{L}(V)$, par :

$$
\omega_{L}(V)=\inf \operatorname{deg}_{L}(Z),
$$

où l'infimum est pris sur l'ensemble des hypersurfaces (irréductibles) de $S$ contenant $V$.

Soit $L$ le fibré associé au plongement standard $\mathbb{G}_{m}^{n} \hookrightarrow \mathbb{P}^{n}$. On obtient une hauteur projective $h_{L}$ sur les points de $\mathbb{G}_{m}^{n}(\overline{\mathbb{Q}})$ et un minimum essentiel $\hat{\mu}_{L}^{\text {ess }}$ sur les sousvariétés de $\mathbb{G}_{m}^{n}(\overline{\mathbb{Q}})$.

Théorème 1.6 (Amoroso, David). Soit $V$ une sous-variété stricte de $\mathbb{G}_{m}^{n}$ de codimension $r$ qui n'est contenue dans aucun translaté d'un sous-tore strict de $\mathbb{G}_{m}^{n}$.

On a :

$$
\hat{\mu}_{L}^{\mathrm{ess}}(V) \geq \frac{c(n)}{\omega_{L}(V)}\left(\log \left(3 \omega_{L}(V)\right)\right)^{-\lambda(r)},
$$


où c(n) est un réel strictement positif et $\lambda(r)=\left(9(3 r)^{r+1}\right)^{r}$.

Dans le cas des variétés abéliennes, on dispose déjà de résultats quantitatifs et inconditionnels, mais la dépendance en le degré n'est pas aussi bonne.

Théorème 1.7 (David, Philippon). Soit A une variété abélienne de dimension $g \geq 2$ définie sur $\overline{\mathbb{Q}}$, principalement polarisée par un fibré L. Si V est une sousvariété stricte de A qui n'est pas translatée d'une sous-variété abélienne, on a:

$$
\hat{\mu}_{L}^{\mathrm{ess}}(V) \geq \frac{\min \left\{1 ; \mathscr{R}_{\mathrm{inj}}\right\}^{2(b+1)}}{2^{11 g^{3}}(g-k+1) \operatorname{deg}_{L}(V)^{2 k(b+1)}},
$$

où $k$ désigne le nombre minimal de copies de $V-V$ dont la somme est une sousvariété abélienne de A, b la dimension de cette sous-variété abélienne et $\mathscr{R}_{\mathrm{inj}}$ la plus petite norme de Riemann d'une période d'une conjuguée de A.

Au numérateur, on voit apparaître le rayon d'injectivité, qui est relié à $h_{L}(A)$ (hauteur projective de l'origine dans le plongement associé à $L^{\otimes 16}$ ) par le lemme «matriciel »de Masser [David et Philippon 2002, lemme 6.8]. Cette minoration est monomiale inverse en le degré, alors que dans le cas torique, elle est linéaire inverse en l'indice d'obstruction (aux facteurs logarithmiques près), ce qui correspond à une minoration en $\operatorname{deg}_{L}(V)^{-1 / \operatorname{codim} V}$.

Remarquons enfin que l'hypothèse du théorème 1.7 ( $V$ n'est pas une translatée de sous-variété abélienne stricte) est plus faible que son analogue torique dans le théorème 1.6 ( $V$ n'est pas incluse dans un translaté de sous-tore strict); cette différence se ressent dès qu'on obtient des résultats comparables au théorème 1.6 et on peut préciser la minoration sous l'hypothèse faible, en faisant intervenir la dimension du plus petit translaté de sous-tore strict contenant $V$ [Amoroso et David 2003, corollaire 1.6].

Résultats. Soit $A$ une variété abélienne définie sur $K$ un corps de nombres, et $L$ un fibré ample et symétrique sur $A$. On considère l'hypothèse suivante sur les idéaux premiers de $\mathrm{O}_{K}$ :

Hypothèse H. Il existe une densité positive d'idéaux premiers de réduction ordinaire pour $A$.

Remarquons que la densité de premiers ordinaires dépend de $K$, mais qu'elle ne dépend pas d'un modèle si $K$ est fixé, car on peut caractériser ces premiers $\mathfrak{p}$ (parmi les premiers de bonne réduction) en regardant la valuation des valeurs propres de l'action d'un élément de Frobenius $F_{\mathfrak{p}}$ sur un module de Tate fixé. De plus, si $A$ vérifie $\mathrm{H}$, les puissances et les quotients de $A$ vérifient encore $\mathrm{H}$. Si $A$ et $A^{\prime}$ sont des variétés abéliennes définies sur $K$ et si les premiers ordinaires pour $A$ et pour $A^{\prime}$ sont de densité 1 , les premiers ordinaires de $A \times A^{\prime}$ sont encore de densité 1. 
Il est d'abord nécessaire de supposer que le $p$-rang est égal au rang de HasseWitt pour trouver de bonnes propriétés métriques $p$-adiques pour $A$, reliées au type de réduction modulo $\mathfrak{p}$, pour $\mathfrak{p}$ un idéal premier de $\mathscr{O}_{K}$ divisant un nombre premier $p$. Des contraintes de nature diophantienne conduisent ensuite à travailler avec des idéaux premiers $\mathfrak{p}$ pour lesquels le $p$-rang vaut 0 ou $g$; en effet, lorsque le $p$-rang chute, les propriétés métriques obtenues sont plus faibles et on a besoin, en contrepartie, d'un grand nombre de points de torsion se réduisant sur 0 modulo $\mathfrak{p}$. Notre méthode fonctionne donc également avec des premiers pour lesquels le rang de Hasse-Witt est nul, mais en regard du corollaire 2.8 de [Ogus 1982], ce cas est très sporadique.

Le présent article et le suivant [Galateau 2008] sont consacrés à la preuve du théorème :

Théorème 1.8. Si A vérifie $\mathbf{H}$, on a la propriété $\mathbf{P}(A)$ suivante. Pour toute sousvariété algébrique $V$ stricte de A qui n'est pas contenue dans le translaté d'une sous-variété abélienne stricte de A :

$$
\hat{\mu}_{L}^{\mathrm{ess}}(V) \geq \frac{C_{L}(A)}{\omega_{L}(V)}\left(\log \left(3 \operatorname{deg}_{L}(V)\right)\right)^{-\lambda(r)},
$$

où $C_{L}(A)$ est un réel strictement positif ne dépendant que de $(A, L)$, et où $\lambda(r)$ est une constante explicite.

Dans ce travail, on établit d'abord les estimations $p$-adiques qui sont au cœur de cette approche, puis on adopte le point de vue de la méthode des pentes en vue de la démonstration du théorème 1.8. La preuve s'achève par un argument de descente, devenu classique dans les problèmes de minoration de hauteur. Cet argument comporte des complications techniques, accentuées encore par la spécificité du cas abélien, qui ont tendance à obscurcir l'articulation des arguments. Il nous a donc semblé intéressant de détailler ici le premier cas non trivial, celui de la codimension 2, pour mieux faire apparaître les idées combinatoires sous-jacentes. On explique en conclusion comment appliquer la descente dans le cas général. Ce travail se poursuit dans [Galateau 2008], qui utilise les estimations $p$-adiques démontrées ici, et donne les détails de la descente en codimension quelconque. Par ailleurs, on a cru utile de donner un autre éclairage sur ce problème en adoptant, dans le second texte, le langage diophantien classique.

L'hypothèse $\mathrm{H}$ est l'objet de la conjecture suivante :

Conjecture 1.9 (Serre). Toute variété abélienne définie sur $\overline{\mathbb{Q}}$ vérifie $H$.

Sous cette conjecture, la propriété $\mathbf{P}(A)$ est valide pour toute variété abélienne $A$ définie sur $\overline{\mathbb{Q}}$. On peut conjecturer [Pink 1998, §7] que les premiers ordinaires sont en densité 1 , quitte à étendre le corps de définition $K$ de $A$.

Pour une courbe elliptique $E$, le résultat est connu. Plus précisément, on sait que la densité de tels idéaux est 1 si $E$ n'est pas CM [Serre 1968, IV, 13], au moins $\frac{1}{2}$ 
si elle est CM. La validité de H a été étendue aux surfaces abéliennes [Ogus 1982, Corollary 2.9]. Notre théorème s'applique alors sans restriction :

Corollaire 1.10. Soit $C$ une courbe algébrique incluse dans une surface abélienne A munie d'un fibré L ample et symétrique. Si C n'est pas le translaté d'une courbe elliptique, on a:

$$
\hat{\mu}_{L}^{\mathrm{ess}}(C) \geq \frac{c_{L}(A)}{\operatorname{deg}_{L}(C)}\left(\log \left(3 \operatorname{deg}_{L}(C)\right)\right)^{-64},
$$

où $c_{L}(A)$ est un réel strictement positif ne dépendant que de $(A, L)$.

Si $A$ est un produit de courbes elliptiques, ou une variété abélienne CM, notre résultat est encore inconditionnel. Des conditions suffisantes pour que $\mathrm{H}$ soit réalisée, portant sur les groupes de monodromie $G_{l}$ (associés à chaque nombre premier $l$ ) de la variété abélienne, ont été données par Noot [1995, §2], puis Pink [1998, §7].

Rappelons que la minoration fine du minimum essentiel permet d'obtenir des résultats en direction des conjectures formulées indépendamment par Bombieri, Masser et Zannier [Bombieri et al. 2007, §5], Zilber [2002] sur les variétés semiabéliennes, puis Pink [2005, Conjectures 1.2 et 1.3] sur les variétés de Shimura mixtes. Pour $S$ un sous-ensemble de $\mathbb{G}_{m}^{n}$, on note :

$$
S_{\varepsilon}=\left\{x y: x \in S, y \in \mathbb{G}_{m}^{n}, h_{L}(y) \leq \varepsilon\right\} .
$$

Le théorème 1.6 est ainsi utilisé dans [Habegger 2009] pour démontrer :

Théorème 1.11 (Habegger). Soit $C$ une courbe algébrique dans $\mathbb{G}_{m}^{n}$ qui n'est pas incluse dans le translaté d'un sous-tore strict. Il existe $\varepsilon>0$ tel que $C \cap \mathcal{H}_{\varepsilon}$ est fini, où :

$$
\mathscr{H}=\bigcup_{\operatorname{codim} H=2} H
$$

la réunion portant sur tous les sous-groupes algébriques de $\mathbb{G}_{m}^{n}$ ayant la codimension prescrite.

Ce théorème généralise à la fois le théorème 2 de [Bombieri et al. 1999] et la propriété de Bogomolov pour les courbes plongées dans les tores. Récemment, Maurin [2008] a démontré la conjecture de Zilber pour une courbe plongée dans un tore, en utilisant le théorème 1.11 et une inégalité de Vojta uniforme.

Théorème 1.12 (Maurin). Soit $C$ une courbe algébrique de $\mathbb{G}_{m}^{n}$ non incluse dans le translaté d'un sous-tore strict par un point de torsion. Alors $C \cap \mathcal{H}$ est fini.

Ce théorème optimise le résultat principal de [Bombieri et al. 1999], qui suppose que $C$ n'est pas incluse dans un translaté de sous-tore strict. Dans le cadre abélien, en utilisant des estimations de type Lehmer sur la hauteur des petits points, on sait essentiellement traiter le cas des variétés abéliennes à multiplication complexe 
[Viada 2003; Rémond et Viada 2003; Ratazzi 2008; Carrizosa 2009]. Viada [2008; 2009] a récemment utilisé le théorème 1.8 pour établir certains cas particuliers de la conjecture de Zilber-Pink sur les variétés abéliennes.

Théorème 1.13 (Viada). Soit $C$ une courbe algébrique incluse dans $E^{g}$, où $E$ est une courbe elliptique et $g \geq 2$. Si $C$ n'est pas incluse dans le translaté d'une sousvariété abélienne stricte par un point de torsion, il existe $\varepsilon>0$ tel que $C(\overline{\mathbb{Q}}) \cap \mathscr{H}_{\varepsilon}$ soit fini.

Plan de l'article. La deuxième partie est consacrée à la démonstration d'une propriété $p$-adique obtenue par l'étude du groupe formel d'une variété abélienne en caractéristique $p$. On commence par faire quelques rappels sur le $p$-rang d'une variété abélienne, puis sur la théorie des schémas en groupes. On obtient ensuite un résultat métrique $p$-adique précis, pour les points de $p$-torsion de $A$ se réduisant sur 0 modulo un idéal premier $\mathfrak{q}$ divisant $p$ dans une extension convenable.

Dans la troisième partie, on rappelle les définitions et résultats généraux de la théorie des pentes. Un premier fait assez inhabituel dans notre application de cette théorie est l'importance des estimations ultramétriques. Dans cette perspective, on utilise une version du théorème des pentes assez précise sur le plan ultramétrique. Puis on introduit les fibrés hermitiens qui seront utiles par la suite et on estime leur pente. La principale difficulté de cette partie réside dans la majoration de la pente maximale du fibré des sections d'un fibré ample sur une sous-variété de $A$ (avec multiplicités), le fibré d'arrivée étant habituellement formé, dans la méthode des pentes, à partir d'un nombre fini de points. Cette majoration est obtenue en suivant une idée figurant dans [Chen 2006]. Les résultats de [Bost et Künnemann 2007] (améliorés par Chen en dimension $\geq 3$ ) sur la pente maximale du produit tensoriel de deux fibrés hermitiens permettent de prendre en compte la multiplicité.

On prend ensuite (quatrième partie) une sous-variété stricte $V$ d'une variété abélienne $A$ qui n'est pas incluse dans un translaté de sous-variété abélienne et on lui associe un fermé de Zariski $X$ en vue de la fin de la preuve; on construit deux espaces vectoriels $E$ et $F$ et un morphisme de restriction entre ces espaces (paramétrés en fonction de $A$ et de l'indice d'obstruction de $X$ ). Puis on fixe les paramètres (degré de l'espace de sections, multiplicités, bornes pour la norme des idéaux premiers utilisés) intervenant dans cette construction et on suppose par l'absurde que le minimum essentiel de $X$ est majoré en fonction de ces paramètres. Dans tout ce paragraphe et les suivants, on travaille avec un plongement étiré, devenu classique dans les travaux diophantiens sur les variétés abéliennes pour passer de la hauteur projective à la hauteur de Néron-Tate.

Dans la cinquième partie, on calcule les rangs et les normes des morphismes susceptibles de rentrer dans l'inégalité des pentes. On écrit ensuite cette inégalité, sous l'hypothèse que le morphisme soit injectif. On parvient rapidement à une contradic- 
tion. À ce stade du travail, on a montré que le minimum essentiel de $X$ est correctement minoré modulo l'injectivité du morphisme. On suppose donc par l'absurde, dans la sixième partie, que le morphisme n'est pas injectif. On commence par appliquer un lemme de zéros très général d'Amoroso et David. L'utilisation de ce lemme est suivie d'une phase de dénombrement et d'un argument de descente, qui permettent d'obtenir une contradiction. Le travail sur l'injectivité du morphisme s'effectue après l'inégalité des pentes parce qu'il comporte une itération (dans la phase de descente) qui nécessite d'avoir déjà écrit cette inégalité. On détaille la descente en codimension $\leq 2$ et on explique en conclusion comment obtenir le cas général.

Constantes. Le théorème 1.8 montre l'existence d'une constante $C_{L}(A)$ ne dépendant que de $(A, L)$, qui apparait dans la minoration du minimum essentiel. Cette constante est explicitable, mais on obtient une borne monomiale inverse en la hauteur de $A$, plus faible à cet égard que le théorème 1.7 (voir le choix de $C_{L}(A)$ dans les dernières lignes de la preuve), et en particulier très éloignée des conjectures formulées dans [David et Philippon 2007]. Au cours de ce travail, on introduira des constantes $c_{1}, \ldots, c_{21}$ ne dépendant que de $(A, L)$. Le choix des paramètres fera intervenir une constante $C_{0}$, dépendant uniquement de $(A, L)$ elle aussi, qui sera prise grande par rapport aux constantes $c_{i}(1 \leq i \leq 21)$. La constante $C_{L}(A)$ s'exprimera alors simplement en fonction de $C_{0}$.

\section{Un lemme clé $p$-adique sur les variétés abéliennes}

Soit $A$ une variété abélienne définie sur $K$ un corps de nombres et soit $\mathscr{P}_{A}$ un ensemble de premiers bien choisi en fonction de $A$. Le but de ce paragraphe est d'établir une inégalité $p$-adique concernant les points de $p$-torsion de $A$, pour $p \in \mathscr{P}_{A}$. On cherche à montrer, pour les variétés abéliennes, un résultat comparable à l'inégalité suivante, vraie pour tout premier $p$, toute racine $p$-ème de l'unité $\xi$ et toute place $v \mid p$ d'un corps de nombres quelconque contenant $\xi$ :

$$
|\xi-1|_{v} \leq p^{-1 / p}
$$

Cette inégalité, conséquence d'une propriété de ramification bien connue sur les corps cyclotomiques [Ireland et Rosen 1990, Proposition 13.2.7], a un analogue satisfaisant sur les courbes elliptiques pour les premiers de bonne réduction ordinaire, s'il n'y a pas de ramification initiale; dans le cas des premiers supersinguliers, on doit remplacer $1 / p$ par $1 / p^{2}$ [Galateau 2007, 2.4.1]. On s'attend donc à ce que le résultat obtenu sur $A$ dépende de la réduction de $A$ modulo un idéal premier $\mathfrak{p}$ de $\mathcal{O}_{K}$. Deux invariants apparaissent naturellement : le rang de la matrice de HasseWitt et son rang stable (ou $p$-rang de la variété). On obtient les meilleurs résultats quand ces deux invariants sont égaux. Ce résultat $p$-adique permettra d'estimer certaines normes ultramétriques apparaissant dans l'inégalité de pentes. 
2A. Le p-rang d'une variété abélienne. On fixe pour ce paragraphe et le suivant un premier $p$ et une variété abélienne $A$ définie sur un corps fini $\mathbb{F}_{q} \subset k:=\overline{\mathbb{F}}_{p}$.

Proposition 2.1. Le groupe $A[p]$ des points de p-torsion sur $\overline{\mathbb{F}}_{p}$ est isomorphe à $(\mathbb{Z} / p \mathbb{Z})^{\alpha}$, où $0 \leq \alpha \leq g$. Cet entier $\alpha$ est appelé p-rang de $A$.

Remarque. Le $p$-rang de $A$ est aussi appelé rang stable de $A$ (en raison de son lien avec la matrice de Hasse-Witt de $A$, explicité infra, 2B). S'il est égal à $g$, la variété $A$ est dite ordinaire.

On introduit maintenant le morphisme de Frobenius relatif, qu'on notera $\mathfrak{F}$. Sur $\operatorname{Spec}(k)$, on définit $\mathfrak{F}$ comme étant l'identité sur l'espace topologique réduit à un point et l'élévation à la puissance $p$ sur $k$. On note ensuite $A^{(p)}$ le tiré en arrière de $A$ par l'action du Frobenius $\mathfrak{F}$ sur $\operatorname{Spec}(k)$. Par construction, ce schéma est une variété abélienne.

Définition 2.2. Le morphisme de Frobenius :

$$
\mathfrak{F}: A \rightarrow A^{(p)} .
$$

est défini par l'élévation à la puissance $p$ sur le faisceau structural.

On vérifie que le Frobenius est une isogénie purement inséparable de degré $p^{g}$. Cette isogénie nous permet de factoriser la multiplication par $p$ sur $A$, notée $[p]$.

Lemme 2.3 [Demazure et Grothendieck 1970, VII A.4]. Il existe une isogénie $\mathfrak{V}: A^{(p)} \rightarrow A$, appelée Verschiebung, telle que $[p]=\mathfrak{V} \circ \mathfrak{F}$. De plus, $\mathfrak{V}$ et $\mathfrak{F}$ sont duales l'une de l'autre au sens suivant: si on note $\hat{A}$ la duale de $A$, on a une décomposition $[p]_{\hat{A}}=\mathfrak{V}_{\hat{A}} \circ \mathfrak{F}_{\hat{A}}$ avec:

$$
\widehat{\mathfrak{V}}=\mathfrak{F}_{\hat{A}} \quad \text { et } \quad \widehat{\mathfrak{F}}=\mathfrak{V}_{\hat{A}} .
$$

Comme le morphisme de Frobenius est purement inséparable, le degré séparable de $\mathfrak{V}$ est égal à $p^{\alpha}$. Si $\alpha=g$, l'isogénie $\mathfrak{V}$ est séparable et sa différentielle en 0 est inversible. On veut relier plus généralement $\alpha$ à la différentielle de $\mathfrak{V}$ en 0 ; ceci nous amène à étudier la structure de schéma en groupe du sous-groupe $A[p]$ de $A$.

2B. Schémas en groupe. On fait ici quelques rappels sur la théorie des schémas en groupes, suivant [Mumford 1974, III].

Définition 2.4. Un schéma en groupe $G$ sur $k$ est un schéma sur $k$ muni d'un morphisme de multiplication $m: G \times G \rightarrow G$, d'un morphisme d'inversion $i$ : $G \rightarrow G$ et d'un élément neutre $e: \operatorname{Spec}(k) \rightarrow G$ vérifiant les axiomes :

$$
\begin{aligned}
m \circ\left(m \times \operatorname{Id}_{G}\right) & =m \circ\left(\operatorname{Id}_{G} \times m\right): G \times G \times G \rightarrow G, \\
m \circ\left(e \times \operatorname{Id}_{G}\right) & =j_{1}: \operatorname{Spec}(k) \times G \rightarrow G, \\
m \circ\left(\operatorname{Id}_{G} \times e\right) & =j_{2}: G \times \operatorname{Spec}(k) \rightarrow G, \\
e \circ \pi=m \circ\left(\operatorname{Id}_{G} \times i\right) & =m \circ\left(i \times \operatorname{Id}_{G}\right): G \rightarrow G,
\end{aligned}
$$


où $\pi: G \rightarrow \operatorname{Spec}(k) ; j_{1}: \operatorname{Spec}(k) \times G \simeq G$ et $j_{2}: G \times \operatorname{Spec}(k)$ sont les isomorphismes canoniques.

Soit $G$ un schéma en groupe. Son algèbre de Lie est le $k$-espace vectoriel des champs de vecteurs invariants par $m$ et elle est munie de la fonction de HasseWitt, qui associe à une dérivation $D$ la dérivation $D^{p}$ ( $p$-ème itérée de $D$ ). C'est une application $\mathbb{F}_{p}$-linéaire (i.e., additive et linéaire sous la multiplication par un élément de $\mathbb{F}_{p}$ ). On définit $\widehat{G}$ le dual (de Cartier) de $G$ d'un schéma en groupe affine $\operatorname{Spec}(R)$ (où $R$ est une $k$-algèbre de type fini) en munissant le dual $R^{*}$ de $R$ d'une comultiplication et d'un idéal d'augmentation par dualité.

On suppose maintenant que $G$ est un schéma en groupe fini et commutatif. On dit que $G$ est de type $l$ si l'espace sous-jacent est constitué d'un seul point, et de type $r$ si $G$ est réduit. On dit que $G$ est de type $(x, y)$ si $G$ est de type $x$ et $\widehat{G}$ est de type $y$. Le schéma $G$ se décompose alors de façon unique en un produit :

$$
G=G_{r, r} \times G_{r, l} \times G_{l, r} \times G_{l, l},
$$

où $G_{x, y}$ est de type $(x, y)$ (pour plus de détails, voir [Mumford 1974, §14]).

Pour le schéma en groupe $A[p]$ qui nous intéresse ici, le type $G_{r, r}$ est trivial car $A[p]$ est de cardinal une puissance de $p$. Plus précisément :

Proposition 2.5. On a l'isomorphisme de schémas en groupes:

$$
A[p] \simeq(\mathbb{Z} / p \mathbb{Z})^{\alpha} \times\left(\mu_{p}\right)^{\alpha} \times A[p]_{l, l},
$$

où $\alpha$ est le $p$-rang de $A, \mu_{p}=\operatorname{Spec}\left(k[X] /\left(X^{p}-1\right)\right)$ et $A[p]_{l, l}$ est de type $(l, l)$.

Démonstration. Soit $n \in \mathbb{N}^{*}$. Compte tenu des structures de groupes de $A\left[p^{n}\right]$ et du dual $\widehat{A\left[p^{n}\right]}$, qui donnent les composantes réduites de ces deux schémas en groupes, et comme le dual du noyau de l'isogénie $[p]$ est le noyau de l'isogénie duale [Mumford 1974, page 143], on a la décomposition :

$$
A\left[p^{n}\right] \simeq\left(\mathbb{Z} / p^{n} \mathbb{Z}\right)^{\alpha} \times\left(\widehat{\mathbb{Z} / p^{n} \mathbb{Z}}\right)^{\beta} \times A\left[p^{n}\right]_{l, l},
$$

pour un certain entier $\beta$ et un schéma en groupe local $A\left[p^{n}\right]_{l, l}$.

L'algèbre de fonctions associée à $\mathbb{Z} / p^{n} \mathbb{Z}$ est son algèbre de groupe et, en notant $X$ l'évaluation en $1 \in \mathbb{Z} / p^{n} \mathbb{Z}$, on voit que l'algèbre duale est isomorphe à :

$$
\operatorname{Spec}\left(k[X] /\left(X^{p^{n}}-1\right)\right) \text {. }
$$

On en déduit que $\widehat{\mathbb{Z} / p^{n} \mathbb{Z}} \simeq \mu_{p^{n}}$. De plus, $\alpha$ et $\beta$ sont permutés par passage de $A$ à $\hat{A}$ et puisqu'il existe une isogénie $f: A \rightarrow \hat{A}$, en notant $K$ le cardinal de son noyau, on a :

$$
f\left(A\left[p^{n}\right]\right) \subset \hat{A}\left[p^{n}\right] \quad \text { donc } \quad p^{n \alpha} \leq K p^{n \beta} .
$$

En faisant varier $n$, on obtient $\alpha \leq \beta$. Mais comme la duale de $\hat{A}$ est isomorphe à $A$ [Mumford 1974, page 132], on obtient $\alpha=\beta$. Le résultat suit en prenant $n=1$. 
Remarque. Les schémas en groupes de type $(l, l)$ sont les plus difficiles à comprendre. Pour plus de détails, voir par exemple [Pink 2004, §16 et §22].

On peut maintenant faire le lien entre la différentielle de $\mathfrak{V}$ en 0 et le $p$-rang :

Proposition 2.6. Soit $\Psi$ la différentielle de $\mathfrak{V}$ en 0 . Le p-rang de A est le rang de $\Psi^{g}$.

Démonstration. On passe aux algèbres de Lie dans la proposition précédente et on observe que l'application linéaire $[p]^{*}$ est la multiplication par $p$, donc est nulle sur Lie $(A)$. On se limite à la partie locale en 0 :

$$
\operatorname{Lie}(A)=\operatorname{Lie}(A[p])=\operatorname{Lie}\left(\mu_{p}\right)^{\alpha} \oplus \operatorname{Lie}\left(A[p]_{l, l}\right) .
$$

En prenant comme base de $\operatorname{Lie}\left(\mu_{p}\right)$ la dérivation $X \partial / \partial X$, on observe que la fonction de Hasse-Witt est l'identité sur $\operatorname{Lie}\left(\mu_{p}\right)^{\alpha}$, alors qu'elle est nilpotente sur la partie locale-locale. De plus, par l'isomorphisme canonique :

$$
\text { Lie } \hat{A} \simeq H^{1}\left(A, O_{A}\right),
$$

la fonction de Hasse-Witt correspond à l'application induite par le Frobenius sur $\mathcal{O}_{A}$ [Mumford 1974, page 148], qui correspond par dualité à la différentielle de $\mathfrak{V}$ sur le tangent en 0 . Il existe donc une décomposition $t_{A}=t_{A, s}+t_{A, n}$ du tangent en 0 laissée stable par $\Psi$ telle que $\Psi_{\mid t_{A, S}}$ soit un isomorphisme et $\Psi_{\mid t_{A, n}}$ soit nilpotente ; de plus, l'espace vectoriel $t_{A, s}$ est de dimension $\alpha$. En itérant $g$ fois l'application $\Psi$, la partie nilpotente s'annule et on en déduit que le $p$-rang est le rang de $\Psi^{g}$.

Remarque. On appelle composante semi-simple de $\Psi$ l'espace vectoriel $t_{A, s}$. Cette composante semi-simple est l'image de $\Psi^{g}$, donc est définie sur $\mathbb{F}_{q}$.

Le $p$-rang d'une variété abélienne n'est pas toujours égal au rang de la matrice de Hasse-Witt. Si on fixe $\alpha \leq g-1$, on peut même montrer [Koblitz 1975, Theorem 7] que sur l'espace de module des variétés abéliennes principalement polarisées de dimension $g$ avec structure de niveau fixée sur $k$, les variétés abéliennes ayant une matrice de Hasse-Witt de rang $g-1$ sont Zariski-denses dans le fermé des variétés abéliennes dont le $p$-rang est plus petit que $\alpha$. Si le $p$-rang est égal à $g-1$ ou $g$, il est automatiquement égal au rang de la matrice de Hasse-Witt.

En général, le $p$-rang et le rang de la matrice de Hasse-Witt sont distincts, et la partie nilpotente fait obstruction pour contrôler efficacement la norme $p$-adique de tous les paramètres.

Hypothèse. On suppose maintenant que le p-rang de A est égal au rang de $\Psi$.

On choisit un système de paramètres $\left(x_{1}, \ldots, x_{g}\right)$ associé à une base de différentielles invariantes, tel que $\left(x_{1}, \ldots, x_{\alpha}\right)$ soit une base de $\operatorname{Im} \Psi^{g}$ (qui est égale à $\operatorname{Im} \Psi)$. Notons $\widehat{O}_{0, A}$ le groupe formel associé à $A$ en 0 sur $\mathbb{F}_{q}$. On a [Hindry et 
Silverman 2000, page 268] :

$$
\widehat{\mathrm{O}}_{0, A} \simeq \mathbb{F}_{q} \llbracket x_{1}, \ldots, x_{g} \rrbracket .
$$

On note encore $\mathfrak{V}:=\left(\mathfrak{V}_{1}, \ldots, \mathfrak{V}_{g}\right)$ le $g$-uplet de séries formelles image de l'isogénie $\mathfrak{V}$ dans le groupe formel. On note aussi $\Phi_{\alpha}$ le morphisme de $\mathbb{F}_{q} \llbracket x_{1}, \ldots, x_{g} \rrbracket$ qui agit sur les paramètres par :

$$
\begin{array}{ll}
x_{i} \rightarrow x_{i} & \text { si } i \leq \alpha, \\
x_{i} \rightarrow x_{i}^{p} & \text { si } i>\alpha .
\end{array}
$$

Corollaire 2.7. Il existe un g-uplet de séries formelles $\mathbf{U}=\left(U_{1}, \ldots, U_{g}\right)$ tel que $d \mathbf{U}$ est inversible et $\mathfrak{V}$ se factorise $: \mathfrak{V}=\mathbf{U} \circ \Phi_{\alpha}$.

Démonstration. Pour tout entier $1 \leq i \leq g$, comme $\mathfrak{V}$ est une isogénie, la forme différentielle $\mathfrak{V}^{*} d x_{i}$ est encore une différentielle invariante, donc se décompose :

$$
\mathfrak{V}^{*} d x_{i}=\sum_{j=1}^{g} \alpha_{i, j} d x_{j},
$$

où les $\alpha_{i, j}$ sont constants et donnés par la $i$-ème colonne de la matrice de $\Psi$ dans la base associée à $\left(x_{1}, \ldots, x_{g}\right)$. On en déduit par intégration que les seuls termes nonnuls dans les $\mathfrak{V}_{i}$ sont les termes linéaires ou des monômes en $\left(x_{1}^{p}, \ldots, x_{g}^{p}\right)$. Par choix de la base, les paramètres $\left(x_{\alpha+1}, \ldots, x_{g}\right)$ sont absents de la partie linéaire. On a donc bien la décomposition voulue. L'application $\Phi_{\alpha}$ est purement inséparable et son degré est le rang de :

$$
k \llbracket x_{1}, \ldots, x_{g} \rrbracket /\left(x_{1}, \ldots, x_{\alpha}, x_{\alpha+1}^{p}, \ldots, x_{g}^{p}\right),
$$

donc :

$$
\operatorname{deg} \Phi_{\alpha}=\operatorname{deg}_{i} \Phi_{\alpha}=p^{g-\alpha} .
$$

En comparant les degrés séparables et inséparables, on voit que $\mathbf{U}$ est séparable, et que sa différentielle est inversible [Lang 2002, VIII, proposition 5.5].

2C. Retour en caractéristique nulle. On traduit maintenant la proposition précédente en un résultat $p$-adique pour les points de torsion d'une variété abélienne définie sur un corps de nombres. Soit donc $A$ une variété abélienne de dimension $g$ définie sur un corps de nombres $K$, munie d'un fibré $L$ ample et symétrique, et soit $\mathscr{A}$ un modèle entier de $A$ sur $\mathbb{O}_{K}$. On peut supposer [Hindry et Silverman 2000, page 105], quitte à considérer $L^{\otimes 3}$, que le fibré $L$ est très ample (et projectivement normal). Rappelons que pour tout nombre premier $p$, il y a $p^{2 g}$ points de $p$-torsion dans $A(\bar{K})$. Pour un idéal premier $\mathfrak{p}$ de $\mathcal{O}_{K}$ de bonne réduction divisant $p$, la fibre spéciale $\mathscr{A}_{\mathfrak{p}}$ ne contient plus que $p^{\alpha}$ points de $p$-torsion, où $\alpha$ est le $p$-rang de la fibre spéciale. On fixe à présent une base $\left(s_{i}\right)_{i}$ de $H^{0}(A, L)$. Par abus de langage, on dira qu'une constante ne dépend que de $A$ si elle dépend de $(A, L)$ et du choix de cette base. 
Précisons d'abord que si q est un idéal premier de $K^{\prime}$ une extension finie de $K$, dont la projection sur $\mathbb{Z}$ est $p$, on choisit la normalisation suivante pour la valuation $\mathfrak{q}$-adique :

$$
|p|_{\mathfrak{q}}=p^{-n_{\mathfrak{q}}}, \quad \text { où } n_{\mathfrak{q}} \text { est le degré local }\left[K_{\mathfrak{q}}^{\prime}: \mathbb{Q}_{p}\right] .
$$

Cette normalisation permet d'écrire plus simplement la formule du produit, et la hauteur d'un morphisme dans l'inégalité de pentes.

Les premiers de réduction ordinaire (i.e., les premiers de bonne réduction pour lesquels le $p$-rang est égal à $g$ ) sont ceux pour lesquels les propriétés métriques sont les meilleures. Dans le cas d'une courbe elliptique $E$, on sait qu'ils sont de densité 1 si $E$ n'est pas à multiplication complexe; et qu'ils sont de densité au moins $\frac{1}{2}$ si $E$ est à multiplication complexe. En dimension supérieure, on ne connaît aucun résultat comparable.

Définition 2.8. Soit 2 un sous-ensemble de l'ensemble $\mathscr{P}$ des idéaux premiers de $0_{K}$. On dit que 2 a une densité naturelle $d$ si le quotient :

$$
\frac{|\{\mathfrak{q} \in \mathscr{2}: \mathbf{N}(\mathfrak{q}) \leq x\}|}{|\{\mathfrak{p} \in \mathscr{P}: \mathbf{N}(\mathfrak{p}) \leq x\}|}
$$

tend vers $d$ quand $x \rightarrow \infty$.

Remarque. La fonction $\mathbf{N}$ est la norme sur les idéaux (définie dans [Samuel 1967, III, 5]).

Dans toute la discussion qui suit, on omet de préciser les ensembles indexateurs, qui sont toujours finis et dépendent de $A$. La loi d'addition de $A$ est donnée sur chaque ouvert affine par des polynômes de bi-degré $(2,2)$ [Lange et Ruppert 1985; David et Philippon 2002, proposition 3.7] dont les coefficients sont de hauteur bornée uniquement en fonction de $A$. On a donc, si on note $\left(x_{k}\right)_{k}$ l'ensemble fini de ces coefficients :

$$
\forall k:\left|x_{k}\right|_{\mathfrak{p}} \leq 1,
$$

sauf pour un nombre fini d'idéaux premiers $\mathfrak{p}$ (ne dépendant que de $A$ ).

Fixons maintenant une base de dérivations algébriques sur $A$. Quitte à prendre des idéaux premiers $\mathfrak{p}$ de $O_{K}$ plus grands qu'une constante ne dépendant que de $A$, cette base de dérivations sera encore une base de dérivations modulo $\mathfrak{p}$. On a fixé une base $\left(s_{i}\right)_{i}$ de $H^{0}(A, L)$ et on note $f_{i}:=s_{i} / s_{0}$ la fonction affine sur l'ouvert de $A$ défini par la non-annulation de $s_{0}$.

Théorème 2.9. Il existe une base de dérivations $\left(\partial_{1}, \ldots, \partial_{g}\right)$ sur A telle que:

$$
\forall(i, j): \partial_{j} f_{i}=\sum_{(k, l)} y_{k, l}^{i, j} f_{k} f_{l},
$$

où les $y_{k, l}^{i, j} \in \overline{\mathbb{Q}}$ sont de hauteur bornée uniquement en fonction de A. 
Démonstration. Le théorème 4.1 de [David 1991] vaut sous l'hypothèse que la polarisation est principale ; le résultat obtenu est alors effectif. L'argument s'adapte sans peine à notre cas. Rappelons-en les étapes.

Soit $\left(\delta_{1}, \ldots, \delta_{g}\right)$ une base de dérivations quelconque en 0 . Le fibré $L$ étant très ample, on peut supposer, quitte à renuméroter, que la matrice $\left(\delta_{k} s_{l}\right)_{1 \leq k, l \leq g}$ est inversible. Il s'agit de la matrice de passage d'une certaine base $\left(\partial_{1}, \ldots, \partial_{g}\right)$ de dérivations en 0 vers $\left(\delta_{1}, \ldots, \delta_{g}\right)$. On a :

$$
s_{0}^{2} \partial_{j}\left(\frac{s_{i}}{s_{0}}\right) \in H^{0}\left(A, L^{\otimes 2}\right),
$$

et comme le plongement associé à $L$ est projectivement normal, on a l'écriture attendue avec les $y_{k, l}^{i, j}$ dans $\mathbb{C}$. Par construction de la base de dérivations, les $y_{k, l}^{i, j}$ sont en fait dans $\overline{\mathbb{Q}}$. Ces coefficients étant en nombre fini, on peut trouver une borne pour leur hauteur ne dépendant que de $A$.

Pour tout idéal premier $\mathfrak{p}$ de $O_{K}$ sauf un nombre fini (ne dépendant que de $A$ ) :

$$
\forall(i, j, k, l):\left|y_{k, l}^{i, j}\right|_{\mathfrak{p}} \leq 1 .
$$

Par abus de langage, on notera encore $\left(\partial_{1}, \ldots, \partial_{g}\right)$ la base de dérivations en 0 qui en résulte. On ne pourra travailler directement avec cette base de dérivations mais on s'y ramènera grâce à la construction suivante.

Lemme 2.10. Il existe un ensemble fini $\left(t_{1, v}, \ldots, t_{g, v}\right)_{v \in \mathcal{V}}$ de systèmes de paramètres en 0 définis sur des ouverts $\left(\mathcal{U}_{v}\right)_{v \in \mathcal{V}}$ tels que $A(\bar{K})=\bigcup_{v \in \mathcal{V}} U_{\nu}$.

Démonstration. Si $x \in A(\bar{K})$ est différent de 0 , il existe un hyperplan passant par $x$ et évitant 0 , défini par l'annulation d'une section $s_{x} \in H^{0}(A, L)$, et un hyperplan évitant à la fois $x$ et 0 , défini par l'annulation d'une section $s_{x}^{\prime} \in H^{0}(A, L)$. En multipliant un système de paramètres en l'origine quelconque $\left(t_{1}, \ldots t_{g}\right)$ par une puissance tensorielle suffisante de $s_{x} / s_{x}^{\prime}$, on obtient un nouveau système de paramètres en 0 défini sur un ouvert $u_{x}$ contenant $x$. On conclut par compacité.

Pour tout $v \in \mathscr{V}$, on définit par dualité une base $\left(\partial_{1, v}, \ldots, \partial_{g, v}\right)$ de dérivations en 0 . Comme $\mathscr{V}$ est fini, pour tout idéal premier $\mathfrak{p}$ de $O_{K}$ sauf un nombre fini, si on note $\left(z_{m}\right)_{m}$ les coefficients de toutes les matrices de passage de $\left(\partial_{1}, \ldots, \partial_{g}\right)$ vers les $\left(\partial_{1, v}, \ldots, \partial_{g, v}\right)_{v \in \mathcal{V}}$ (qui sont définis sur une extension finie) :

$$
\left.\forall m \forall \mathfrak{q}|\mathfrak{p}:| z_{m}\right|_{\mathfrak{q}} \leq 1
$$

Les premiers de mauvaise réduction pour $A$ sont en nombre fini, ainsi que les premiers de $\mathbb{Z}$ se ramifiant dans $\mathscr{O}_{K}$. On pose $\mathscr{P}_{A, 0}$ l'ensemble des premiers $\mathfrak{p}$ de $\mathscr{O}_{K}$ de bonne réduction, vérifiant (2-1), (2-2) et (2-3), tels que la base de dérivations algébriques soit encore une base sur $\mathscr{A}_{\mathfrak{p}}$, tels que si $(p)=\mathfrak{p} \cap \mathbb{Z}$, on a $e_{\mathfrak{p} / p}=1$, et 
enfin, tels que :

$$
\sum_{\substack{p=\mathfrak{p}_{\mathbb{Z}} \\ \mathfrak{p} \in \mathscr{P}_{A, 0}}} \frac{1}{p^{2}} \leq \frac{1}{3} .
$$

Comme la même somme indexée par $\mathbb{N}^{*}$ converge, il suffit d'exclure un ensemble fini (absolu) de premiers pour que cette condition soit vérifiée. L'ensemble $\mathscr{P}_{A, 0}$ est de densité naturelle égale à 1 , et sa construction ne dépend que de $A$ et $K$.

On fait l'hypothèse suivante sur $\mathscr{A}$ :

Hypothèse $\mathbf{H}^{\prime}$. Il y a une densité $c_{0}>0$ d'idéaux premiers $\mathfrak{p}$ pour lesquels le p-rang de $\mathscr{A}_{\mathfrak{p}}$ est égal au rang de la matrice de Hasse-Witt.

Par le principe des tiroirs de Dirichlet, il existe un entier $k$ tel que la densité naturelle d'idéaux premiers de $\mathscr{P}_{A, 0}$ vérifiant $\mathrm{H}^{\prime}$ et pour lesquels la fibre spéciale a un $p$-rang égal à $k$ est supérieure ou égale à $c_{0} /(g+1)$. On choisit un tel entier et on le note $\alpha$. On note $\mathscr{P}_{A}$ l'ensemble des idéaux premiers $\mathfrak{p}$ de $\mathscr{P}_{A, 0}$ en lesquels la variété $\mathscr{A}_{\mathfrak{p}}$ a un $p$-rang égal à $\alpha$. De plus, quitte à diviser la densité de cet ensemble par $[K: \mathbb{Q}]$, on peut supposer que deux idéaux premiers distincts de $\mathscr{P}_{A}$ ont des projections distinctes sur $\mathbb{Z}$.

Soit $\mathfrak{p} \in \mathscr{P}_{A}$ un idéal premier et $(p)=\mathfrak{p} \cap \mathbb{Z}$. Par choix de $\mathscr{P}_{A}$, la fibre spéciale $\mathscr{A}_{\mathfrak{p}}$ est lisse. Soit $\Psi_{\mathfrak{p}}$ la différentielle du Verschiebung sur $\mathscr{A}_{\mathfrak{p}}$. Par la discussion du paragraphe précédent, on peut trouver une base de paramètres en l'origine de $A$ :

$$
t_{\mathfrak{p}, 1}, \ldots, t_{\mathfrak{p}, g}
$$

(i.e., dont la projection est une base de $\mathfrak{m}_{0} / \mathfrak{m}_{0}^{2}$, où $\mathfrak{m}_{0}$ est l'idéal maximal correspondant à l'origine de $A$ ) telle que son image par réduction modulo $\mathfrak{p}$ :

$$
\tilde{t}_{\mathfrak{p}, 1}, \ldots, \tilde{t}_{\mathfrak{p}, g}
$$

soit encore une base de paramètres algébriques, avec :

$$
\begin{aligned}
\operatorname{Im} \Psi_{\mathfrak{p}}^{g} & =\operatorname{Vect}\left(\tilde{t}_{\mathfrak{p}, 1}, \ldots, \tilde{t}_{\mathfrak{p}, \alpha}\right), \\
\operatorname{Ker} \Psi_{\mathfrak{p}}^{g} & =\operatorname{Vect}\left(\tilde{t}_{\mathfrak{p}, \alpha+1}, \ldots, \tilde{t}_{\mathfrak{p}, g}\right) .
\end{aligned}
$$

Ce système de paramètres est défini sur un certain ouvert affine $\boldsymbol{U}_{\mathbf{t}_{\mathfrak{p}}}$. On note $\boldsymbol{O}_{\mathfrak{p}}$ l'anneau de valuation associé à p. Il lui correspond par tensorisation :

$$
\mathscr{A}_{\mathfrak{O}_{\mathfrak{p}}}:=\mathscr{A} \times{ }_{\operatorname{Spec} O_{K}} \operatorname{Spec} O_{\mathfrak{p}},
$$

et la section nulle $\varepsilon_{\mathfrak{p}}$. On note $\hat{\mathscr{A}}_{\widehat{O}_{\mathfrak{p}}}$ le complété le long de $\varepsilon_{\mathfrak{p}}$ de $\mathscr{A}_{\Theta_{\mathfrak{p}}}$. La multiplication par $[p]$ est donnée sur le groupe formel par un $g$-uplet de séries formelles

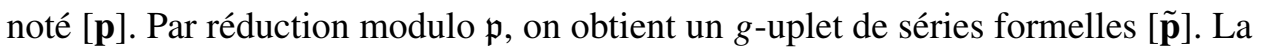
décomposition $[p]_{\mathfrak{p}}=\mathfrak{V}_{\mathfrak{p}} \circ \mathfrak{F}_{\mathfrak{p}}$ de la multiplication par $p$ sur la fibre spéciale induit une décomposition :

$$
[\tilde{\mathbf{p}}]\left(\tilde{\mathbf{t}}_{\mathfrak{p}}\right)=\mathfrak{V}_{\mathfrak{p}}\left(\tilde{\mathbf{t}}_{\mathfrak{p}}^{p}\right),
$$

où $\tilde{\mathbf{t}}_{\mathfrak{p}}^{p}=\left(\tilde{t}_{\mathfrak{p}, 1}^{p}, \ldots, \tilde{t}_{\mathfrak{p}, g}^{p}\right)$ est l'image de $\tilde{\mathbf{t}}_{\mathfrak{p}}$ par le Frobenius. 
Proposition 2.11. Si $P \in U_{t_{\mathfrak{p}}}$ est un point de p-torsion se réduisant sur 0 modulo $\mathfrak{q}$, pour une place $\mathfrak{q} \mid \mathfrak{p}$ dans un corps de définition de $P$, on a:

$$
\begin{aligned}
& \forall 1 \leq i \leq \alpha:\left|t_{\mathfrak{p}, i}(P)\right|_{\mathfrak{q}} \leq p^{-n_{\mathfrak{q}} / p}, \\
& \forall \alpha<i \leq g:\left|t_{\mathfrak{p}, i}(P)\right|_{\mathfrak{q}} \leq p^{-n_{\mathfrak{q}} / p^{2}} .
\end{aligned}
$$

Démonstration. Soit $P \in \mathcal{U}_{\mathfrak{t}_{\mathfrak{p}}}$ se réduisant sur 0 modulo $\mathfrak{q}$, pour $\mathfrak{q} \mid \mathfrak{p}$ dans un corps de définition de $P$. On sait déjà :

$$
\forall 1 \leq i \leq g:\left|t_{\mathfrak{p}, i}(P)\right|_{\mathfrak{q}}<1 .
$$

De plus, le morphisme [ $p$ ] en $P$ est donné par le $g$-uplet de séries formelles [p] appliquées au système de paramètres [Hindry et Silverman 2000, page 272]. On en déduit que $[\mathbf{p}] \circ \mathbf{t}_{\mathfrak{p}}(P)=0$.

D'après le corollaire 2.7, on a une factorisation $\mathfrak{V}_{\mathfrak{p}}=\mathbf{U}_{\mathfrak{p}} \circ \Phi_{\alpha, \mathfrak{p}}$, et la différentielle de $\mathbf{U}_{\mathfrak{p}}$ est inversible. En utilisant la réduction modulo $\mathfrak{p}$ de [p] et les propriétés de base d'une loi de groupe formel (rappelées dans [Hindry et Silverman 2000, page 269]), on voit que [p] est donné par :

$$
[\mathbf{p}]\left(\mathbf{t}_{\mathfrak{p}}\right)=p \mathbf{t}_{\mathfrak{p}}+\mathbf{G}\left(\mathbf{t}_{\mathfrak{p}}\right)+\mathbf{H} \circ \Phi_{\alpha}\left(\mathbf{t}_{\mathfrak{p}}^{p}\right) .
$$

Le $g$-uplet de séries formelles $\mathbf{G}$ a ses coefficients dans $\mathfrak{p} \widehat{O}_{\mathfrak{p}}$ et ses premiers termes sont quadratiques; le $g$-uplet $\mathbf{H}$ a ses coefficients inversibles modulo $\mathfrak{p}$ et sa différentielle est inversible dans $\mathcal{O}_{\mathfrak{p}}$. Soit $i_{0} \in[1, g]$ tel que $\left|t_{\mathfrak{p}, i_{0}}(P)\right|_{\mathfrak{q}}$ soit maximal. En inversant la différentielle, et par choix de $i_{0}$, on obtient :

$$
t_{\mathfrak{p}, i_{0}}(P)^{p^{n_{i}}} \in \mathfrak{p} \mathfrak{O}_{\mathfrak{q}},
$$

où $n_{i_{0}}=1$ si $i_{0} \leq \alpha$ et $n_{i_{0}}=2$ sinon. Comme l'indice de ramification $e_{\mathfrak{p} / p}$ vaut 1 et par définition de $i_{0}$, on en déduit que pour tout $i \leq g$ :

$$
\left|t_{\mathfrak{p}, i}(P)\right|_{\mathfrak{q}} \leq p^{-n_{\mathfrak{q}} / p^{2}}
$$

On est maintenant assuré que les contribution non-linéaires dans $\mathbf{H} \circ \Phi_{\alpha}\left(\mathbf{t}_{\mathfrak{p}}^{p}\right)$ ont une norme $\mathfrak{q}$-adique $\leq 1$. On obtient donc, cette fois, pour tout $i \leq \alpha$ :

$$
t_{\mathfrak{p}, i}(P)^{p} \in \mathfrak{p} \mathfrak{O}_{\mathfrak{q}},
$$

et la proposition est entièrement démontrée.

Remarque. Puisque la ramification initiale $e_{\mathfrak{p} / p}$ est égale à 1 et le groupe des points de $p$-torsion se réduisant sur 0 modulo $\mathfrak{q}$ est galoisien de cardinal inférieur à $p^{2 g-\alpha}$, le théorème de Raynaud [1974, corollaire 3.4.4] donne, pour $1 \leq i \leq g$ :

$$
\left|t_{\mathfrak{p}, i}(P)\right|_{\mathfrak{q}} \leq p^{-n_{\mathfrak{q}} / p^{(2 g-\alpha)}} .
$$


On suppose maintenant que $\alpha=g$. Soit $\left(t_{1}, \ldots, t_{g}\right)$ une base de paramètres en 0 dont la réduction modulo $\mathfrak{p}$ est encore une base, et bien définie sur un certain ouvert affine $\boldsymbol{U}_{\mathbf{t}}$.

Corollaire 2.12. Si $P \in U_{t}$ est un point de p-torsion se réduisant sur 0 modulo $\mathfrak{q}$, pour une place $\mathfrak{q} \mid \mathfrak{p}$ dans un corps de définition de $P$ :

$$
\forall 1 \leq i \leq g:\left|t_{i}(P)\right|_{\mathfrak{q}} \leq p^{-n_{\mathfrak{q}} / p}
$$

\section{Théorie des pentes}

Dans cette partie, on commence par définir les objets qui apparaissent dans la théorie des pentes, puis on donne les inégalités de pentes dont on se servira par la suite. On finit par estimer les pentes de fibrés qui apparaîtront dans la suite de ce travail.

3A. Définitions et inégalité de pentes. Le formalisme des pentes a été introduit par Bost [1996b] et s'est développé dans la littérature diophantienne depuis une dizaine d'années. Pour des détails et des exemples d'applications de la théorie des pentes, on renvoie par exemple aux articles de Bost [1996b; 2001] ou à l'article très complet de Gaudron [2006]. Le but de cette partie est donc d'écrire une inégalité de pentes. Sous sa forme basique, celle-ci compare les pentes de deux $\mathfrak{O}_{K}$-modules hermitiens s'il existe un morphisme $\phi$ injectif entre eux. On va donc définir le degré arithmétique d'un fibré vectoriel hermitien, puis sa pente, sa pente maximale, et la hauteur d'un morphisme de fibrés.

On note dans ce paragraphe $S=\operatorname{Spec}\left(O_{K}\right), S_{0}$ l'ensemble des points fermés de $S$ et $S_{\infty}$ l'ensemble des places archimédiennes de $\mathcal{O}_{K}$; on note enfin $M(K)=S_{0} \cup S_{\infty}$ l'ensemble des places de $K$. Un fibré vectoriel $\mathscr{E}$ sur $S$ est constant, ce qui mène à la définition suivante :

Définition 3.1. Un fibré vectoriel hermitien sur $S$ est un $\mathscr{O}_{K}$-module $\mathscr{E}$ de type fini muni d'une collection $\left\{\|\cdot\|_{v}\right\}_{v \in S_{\infty}}$ telle que pour tout $v \in S_{\infty},\|\cdot\|_{v}$ soit une norme hermitienne sur le $K_{v}$-espace vectoriel $\mathscr{E}_{v}=\mathscr{E} \otimes K_{v}$, invariante par conjugaison complexe.

On notera $\overline{\mathscr{E}}$ le fibré $\left(\mathscr{E},\left\{\|\cdot\|_{v}\right\}\right)$, et $\mathscr{E}_{K}$ (resp. $\left.\mathscr{E}_{v}\right)$ l'espace vectoriel $\mathscr{E}_{\mathscr{E}} \otimes_{\mathfrak{O}_{K}} K$ (resp. $\mathscr{E} \otimes \otimes_{\mathcal{O}_{K}} K_{v}$ pour $v \in S$ ).

Définition 3.2. Soit $\overline{\mathscr{E}}$ un $\mathscr{O}_{K}$-fibré hermitien de rang 1 et $s$ un élément non nul de $\mathscr{E}$. Le degré arithmétique (ou arakélovien) normalisé de $\mathscr{E}$ est :

$$
\widehat{\operatorname{deg}} \overline{\mathscr{E}}=\frac{1}{[K: \mathbb{Q}]}\left(\log \#\left(\mathscr{E} / s \mathbb{O}_{K}\right)-\sum_{v \in S_{\infty}} \log \|s\|_{v}\right) .
$$


Si $\overline{\mathscr{E}}$ est un $\mathscr{O}_{K}$-fibré hermitien de rang $r$, on pose :

$$
\widehat{\operatorname{deg}} \overline{\mathscr{E}}=\widehat{\operatorname{deg}} \operatorname{det} \overline{\mathscr{E}},
$$

où les normes sur le déterminant sont celles obtenues par puissance tensorielle et quotient à partir de celles de $\mathscr{E}$.

Remarques. La formule du produit montre que cette définition ne dépend pas du choix de $s$. Si $K=\mathbb{Q}$, le degré d'Arakelov est l'opposé du logarithme du covolume de $\mathscr{E}$ vu comme réseau de $\mathscr{E} \otimes_{\mathbb{Z}} \mathbb{R}$ (voir [Bost et al. 1994, (2.1.13)] pour le cas général).

Définition 3.3. Soit $\overline{\mathscr{E}}$ un fibré hermitien de rang non nul. On définit sa pente par :

$$
\hat{\mu}(\overline{\mathscr{E}})=\frac{\widehat{\operatorname{deg}} \overline{\mathscr{E}}}{\operatorname{rg} \mathscr{E}} .
$$

Les pentes des sous-modules de $\mathscr{E}$ sont bornées (par l'inégalité d'Hadamard), ce qui justifie la définition :

Définition 3.4. La pente maximale de $\overline{\mathscr{E}}$ est définie par :

$$
\hat{\mu}_{\max }(\overline{\mathscr{\mathscr { C }}})=\max \hat{\mu}(\overline{\mathscr{F}}),
$$

où $\mathscr{F}$ décrit l'ensemble des sous-fibrés non-nuls de $\mathscr{E}$ munis des métriques déduites de celles de $\mathscr{E}$ par restriction.

Soit $\phi$ un morphisme entre deux $\mathscr{O}_{K}$-fibrés $\mathscr{E}$ et $\mathscr{F}$. Si ces fibrés sont hermitiens, pour toute place $v \in M(K)$, on note $\|\phi\|_{v}$ la norme d'opérateur du morphisme $\phi: \mathscr{E}_{v} \rightarrow \mathscr{F}_{v}$. On a donc :

$$
\|\phi\|_{v}=\sup _{\substack{x \in \mathscr{E}_{v} \\ x \neq 0}} \frac{\|\phi(x)\|_{v}}{\|x\|_{v}} .
$$

Définition 3.5. Si $\phi$ est un morphisme entre deux $\mathscr{O}_{K}$-fibrés hermitiens $\overline{\mathscr{E}}$ et $\overline{\mathscr{F}}$, on appelle hauteur de $\phi$ :

$$
h(\phi)=\frac{1}{[K: \mathbb{Q}]} \sum_{v \in M(K)} \log \|\phi\|_{v} .
$$

On est en mesure d'écrire une première inégalité de pentes :

Lemme 3.6. Si le morphisme $\phi: \mathscr{E}_{K} \rightarrow \mathscr{F}_{K}$ est injectif :

$$
\widehat{\operatorname{deg}} \overline{\mathscr{E}} \leq \operatorname{rg} \mathscr{E}\left(\hat{\mu}_{\max }(\overline{\mathscr{F}})+h(\phi)\right) .
$$

Démonstration. C'est une conséquence de l'inégalité d'Hadamard; on renvoie à [Chen 2006, page 40], pour plus de détails (où la même convention est faite sur les normes ultramétriques). 
On utilise généralement une version filtrée de cette inégalité. Soit $\phi: \mathscr{E}_{K} \rightarrow \mathscr{F}_{K}$ une application $K$-linéaire injective. On suppose qu'il existe une filtration d'espaces vectoriels :

$$
\{0\}=\mathscr{F}_{K, N+1} \subset \cdots \subset \mathscr{F}_{K, 0}=\mathscr{F}_{K} .
$$

et que les quotients $\mathscr{G}_{K, i}=\mathscr{F}_{K, i} / \mathscr{F}_{K, i+1}$ sont les tensorisations avec $K$ de fibrés hermitiens $\overline{\mathscr{G}}_{i}$ sur Spec $\mathscr{O}_{K}$. On définit une filtration sur $\mathscr{E}_{K}$ par image réciproque :

$$
\mathscr{\mathscr { E }}_{K, i}=\phi_{K}^{-1}\left(\mathscr{F}_{K, i}\right),
$$

et on considère l'application linéaire naturellement induite sur le quotient :

$$
\phi_{i}: \mathscr{E}_{K, i} \rightarrow \mathscr{G}_{K, i} .
$$

Lemme 3.7. Si $\phi$ est injective, on a l'inégalité:

$$
\widehat{\operatorname{deg}} \overline{\mathscr{E}} \leq \sum_{i=0}^{N} \operatorname{dim}\left(\mathscr{E}_{K, i} / \mathscr{E}_{K, i+1}\right)\left(\hat{\mu}_{\max }\left(\overline{\mathscr{G}}_{i}\right)+h\left(\phi_{i}\right)\right) .
$$

Cette version est particulièrement utile dans une preuve de transcendance : la filtration correspond aux différents ensembles et ordres d'annulation d'une fonction auxiliaire.

3B. Quelques fibrés hermitiens. On peut maintenant préciser les fibrés hermitiens auxquels on compte appliquer la méthode des pentes. Rappelons que $A$ est une variété abélienne munie d'un fibré $L$ ample et symétrique, et définie sur un corps de nombres $K$.

3B1. Tangent et espace symétrique. Quitte à prendre une extension finie de $K$ ne dépendant que de $A$, on suppose que $A$ admet réduction semi-abélienne sur $K$. Il existe donc un modèle semi-abélien $\pi: \mathscr{A} \rightarrow$ Spec $0_{K}$. On note

$$
\varepsilon: \operatorname{Spec} \mathrm{O}_{K} \rightarrow \mathscr{A}
$$

la section nulle et on pose :

$$
t_{\mathscr{A}}:=\varepsilon^{*} T_{\mathscr{A} / \operatorname{Spec} \mathscr{O}_{K}},
$$

où $T_{\mathscr{A} / \mathrm{Spec} \mathscr{O}_{K}}$ est le fibré tangent de $\mathscr{A}$ sur $\mathscr{O}_{K}$. On a une structure de $\mathscr{O}_{K}$-module de type fini sur $t_{\mathscr{A}}$, qui est un sous-module de l'espace vectoriel tangent de $A$ à l'origine. Si $\sigma$ est une place complexe de $K$, il existe un isomorphisme $t_{\mathscr{A}} \otimes_{\sigma} \mathbb{C} \simeq t_{\mathfrak{A}_{\sigma}}(\mathbb{C})$ et la forme de Riemann $\omega$ associée au fibré ample $L$ induit une forme hermitienne $\omega_{\sigma} \in \bigwedge^{1,1} t_{\mathscr{A}_{\sigma}}^{\vee}(\mathbb{C})$. Si $\omega_{\sigma}$ s'écrit sous la forme :

$$
\omega_{\sigma}=\frac{i}{2} \sum_{1 \leq h, l \leq g} a_{h, l} f_{h}^{*} \wedge \overline{f_{l}^{*}}
$$

où $\left(f_{1}^{*}, \ldots, f_{g}^{*}\right)$ est la base duale d'une base $\left(f_{1}, \ldots, f_{g}\right)$ de $t_{\mathscr{A}_{\sigma}}(\mathbb{C})$, on pose, pour 
$\left(z_{1}, \ldots, z_{g}\right) \in \mathbb{C}^{g}:$

$$
\left\|\sum_{i=1}^{g} z_{i} f_{i}\right\|_{\sigma}^{2}=\sum_{\substack{1 \leq h \\ l \leq g}} a_{h, l} z_{h} \overline{z_{l}} .
$$

Ces métriques font de $t_{\mathscr{A}}$ un fibré vectoriel hermitien et cette structure se transporte par dualité à $t_{\mathscr{A}}^{\vee}$.

On sait calculer explicitement la pente de $\overline{t_{\mathscr{A}}}$ en fonction de la hauteur de Faltings de $A$, de la dimension de l'espace des sections de $L$ sur $A$, et de la dimension de $A$ [Gaudron 2006, proposition 4.7]. On se donne ici une constante $c_{4}>0$ ne dépendant que de $A, L$ telle que :

$$
\hat{\mu}_{\max }\left(\overline{t_{\mathscr{A}}^{\vee}}\right) \leq c_{4} .
$$

On a construit une base du tangent ( $\operatorname{sur} \mathscr{O}_{K}$ ) dans laquelle les propriétés $\mathfrak{p}$-adiques de $A$ sont lisibles, pour un grand nombre de premiers $\mathfrak{p}$ de $O_{K}$. On souhaiterait pouvoir majorer, en général, la pente maximale associée à des sous-modules du tangent de rang maximal. Soit $\mathscr{W}$ un sous-module de $t_{\mathscr{A}}$ de rang $g$ engendré par des vecteurs $\left(e_{1}, \ldots, e_{g}\right)$; on munit ce module des métriques hermitiennes (issues de la forme de Riemann) de $t_{\mathscr{A}}$ par restriction. On peut majorer la pente maximale de $\overline{W^{\vee}}$ en fonction de la hauteur d'une base de $\mathcal{W}$. Soit $c(\mathcal{W}) \geq 1$ tel qu'il existe une base de $\mathcal{W}$ formée d'éléments dont toutes les coordonnées sont dans $\mathbb{O}_{K}$ et de hauteur plus petite que $c(\mathscr{W})$.

Lemme 3.8. La pente maximale de $\overline{\mathscr{W}^{\vee}}$ vérifie:

$$
\hat{\mu}_{\max }\left(\overline{W^{\vee}}\right) \leq c_{6} c(\mathcal{W}),
$$

pour une constante $c_{6}>0$ ne dépendant que de $(A, L)$.

Démonstration. La valeur absolue du degré normalisé de $\mathcal{W}$ est majorée, à partir de [Bost et al. 1994, (2.1.13)], de l'inégalité d'Hadamard et en prenant une base de $O_{K}$ d'éléments dont la hauteur est bornée par $c(\mathscr{W})$ :

$$
|\widehat{\operatorname{deg}} \bar{W}| \leq c_{5} c(W),
$$

pour une constante $c_{5}$ ne dépendant que de $g$ et de $K$. En effet, pour les vecteurs à coordonnées dans $\bigcirc_{K}$, il n'y a pas de contribution ultramétrique dans l'expression de la hauteur, et les contributions archimédiennes sont comparables à la norme $L^{2}$ sortant de l'inégalité d'Hadamard. Le lemme se déduit immédiatement de cette inégalité et de [Gaudron 2006, (41)].

Pour passer aux dérivées d'ordre supérieur, on doit comprendre comment la pente maximale se comporte avec les puissances symétriques. Soit $\overline{\mathscr{E}}$ un fibré her-

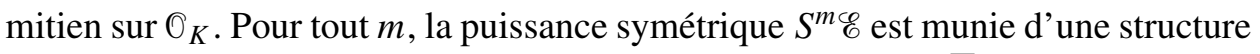
hermitienne par produit tensoriel puis projection; on note $S^{m \overline{\mathscr{E}}}$ le fibré hermitien ainsi obtenu. 
Lemme 3.9 [Graftieaux 2001, appendice]. Pour $\overline{\mathscr{E}}$ un fibré hermitien de rang $g$ :

$$
\hat{\mu}_{\max }\left(S^{m}(\overline{\mathscr{\mathscr { C }}})\right) \leq m\left(\hat{\mu}_{\max }(\overline{\mathscr{\mathscr { C }}})+2 g \log g\right) .
$$

La combinaison des deux derniers lemmes donne la proposition suivante.

Proposition 3.10. On $a$ :

$$
\hat{\mu}_{\max }\left(S^{m}\left(\overline{W^{\vee}}\right)\right) \leq c_{7} m c(\mathcal{W}),
$$

pour une constante $c_{7}$ ne dépendant que de $(A, L)$.

3B2. L'espace des sections d'un fibré ample sur la variété abélienne. La variété abélienne $A$ est munie de $L$ un fibré ample et symétrique, et on note $H^{0}(A, L)$ l'espace des sections sur ce fibré. Si $s \in H^{0}(A, L)$, on peut définir une métrique à l'aide de la fonction $\theta$ associée à $s$. Pour $x=\exp _{A}(z)$ et $z \in t_{A}$, on pose :

$$
\|s(x)\|:=e^{-(\pi / 2)\|z\|^{2}}|\theta(z)| .
$$

Il existe alors, grâce à [Bost 1996a, §4.3], un modèle de $(A, L)$, appelé modèle de Moret-Bailly et noté $(\mathscr{A}, \overline{\mathscr{L}}, 0)$, constitué d'un schéma abélien :

$$
\pi: \mathscr{A} \rightarrow \operatorname{Spec} O_{K}
$$

et d'un fibré hermitien $\overline{\mathscr{L}}$ sur $\mathscr{A}$ vérifiant notamment les propriétés suivantes :

- Il existe un isomorphisme de variétés abéliennes sur $\overline{\mathbb{Q}}: i: A \rightarrow \mathscr{A}_{\overline{\mathbb{Q}}}$.

- Il existe un isomorphisme de fibrés en droites sur $A: i^{*} \mathscr{L}_{\overline{\mathbb{Q}}} \rightarrow L$.

- L'origine de $A$ se relève en une section $\varepsilon: \operatorname{Spec} O_{K} \rightarrow \mathscr{A}$.

- Pour toute place $\sigma$ archimédienne de $K$, la métrique sur $\mathscr{L}_{\sigma} \mathbb{C}$ est la métrique cubiste définie plus haut.

On note $\mathscr{E}$ le $\mathscr{O}_{K}$-module $H^{0}(\mathscr{A}, \mathscr{L})$ et on le munit des métriques hermitiennes suivantes aux places archimédiennes : pour tout plongement $\sigma: K \hookrightarrow \mathbb{C}$ et $s \in$ $\mathscr{E} \otimes_{\sigma} \mathbb{C} \simeq H^{0}\left(\mathscr{A}_{\sigma}, \mathscr{L}_{\sigma}\right)$, on pose :

$$
\|s\|_{L^{2}, \sigma}=\left(\int_{\mathscr{A}_{\sigma}(\mathbb{C})}\|s(x)\|^{2} d \mu(x)\right)^{1 / 2},
$$

où $d \mu$ est la mesure de Haar de masse totale égale à 1 sur $\mathscr{A}_{\sigma}(\mathbb{C})$. On sait que $\overline{\mathscr{E}}$ est semi-stable : sa pente est égale à sa pente maximale [Bost 1996b, 4.2].

Proposition 3.11 [Moret-Bailly 1990; Bost 1996a]. La pente de $\overline{\mathscr{E}}$ est donnée par la formule suivante:

$$
\hat{\mu}(\overline{\mathscr{E}})=-\frac{1}{2} h_{F}(A)+\frac{1}{4} \log \frac{\chi(A, L)}{(2 \pi)^{g}} ;
$$

où $h_{F}(A)$ est la hauteur de Faltings de A et $\chi(A, L)=\operatorname{deg}_{L}(A) / g$ ! désigne la caractéristique d'Euler-Poincaré de A. 
3B3. Pente maximale et sous-variétés. Soit $X$ un fermé de Zariski équidimensionnel de $A$, de dimension $d$. On note $\mathscr{X}$ l'adhérence de Zariski de $X$ dans le modèle $\mathscr{A}$ de $A$ qu'on s'est donné précédemment. Soit $W$ un sous-module du tangent $t_{\mathscr{A}}$, muni de la restriction des métriques hermitiennes provenant de la forme de Riemann. Aux places archimédiennes, on munit :

$$
\mathscr{L}^{\otimes M} \otimes S^{m}\left(W^{\vee}\right)
$$

de la norme obtenue par produit tensoriel de la norme cubiste sur $\mathscr{L} \otimes_{\sigma} \mathbb{C}$ et de la norme symétrique sur $S^{m}\left(W^{\vee}\right) \otimes_{\sigma} \mathbb{C}$, et on munit le $\mathcal{O}_{K}$-fibré :

$$
\mathscr{H}_{m}^{M}:=H^{0}\left(\mathscr{A}, \mathscr{L}^{\otimes M} \otimes S^{m}\left(\mathcal{W}^{\vee}\right)\right)_{\mid \mathscr{L}} \subset H^{0}\left(\mathscr{X}, \mathscr{L}^{\otimes M} \otimes S^{m}\left(\mathcal{W}^{\vee}\right)\right)
$$

de la métrique de Löwner $\|\cdot\|_{L, \sigma}$ associée à la norme du sup, pour toute place archimédienne $\sigma$. Il s'agit d'une norme hermitienne proche de la norme du sup dans le sens suivant (voir [Gaudron 2008, 2.2] pour le cas euclidien) :

$$
\forall x \in \mathscr{H}_{m}^{M} \otimes \mathbb{C}:\|x\|_{L, \sigma} \leq\|x\|_{\text {sup }, \sigma} \leq \sqrt{2 \operatorname{rg} \mathscr{H}_{m}^{M}}\|x\|_{L, \sigma} .
$$

On souhaite majorer convenablement la pente maximale de $\overline{\mathcal{H}_{m}^{M}}$. On étudie d'abord le cas $m=0$. On notera $h_{L}(V)$ la hauteur d'une variété $V$ telle qu'elle est définie dans [Bost et al. 1994, partie 3].

Proposition 3.12. Il existe une constante explicite $c_{8}$ ne dépendant que de $\mathrm{K}$ telle qu'on ait la majoration suivante pour la pente maximale de $\overline{\mathcal{H}_{0}^{M}}$ :

$$
\hat{\mu}_{\max }\left(\overline{\mathscr{H}_{0}^{M}}\right) \leq M \frac{h_{\bar{L}}(X)}{\operatorname{deg}_{L}(X)}+d \log (M+d)+\log \operatorname{deg}_{L}(X)+c_{8} .
$$

Démonstration. On commence par majorer la pente maximale en introduisant la plus petite norme $\varepsilon\left(\overline{\mathcal{H}_{0}^{M}}\right)$ (hermitienne sur la somme orthogonale des $\mathscr{H}_{0}^{M} \otimes_{\sigma} \mathbb{C}$ ) d'un élément non-nul du réseau $\mathscr{H}_{0}^{M}$; voir [Bost et Künnemann 2007, inégalité (3.24) et la majoration de la fonction $\psi$ page 35]. On a :

$$
\hat{\mu}_{\max }\left(\overline{\mathscr{H}_{0}^{M}}\right) \leq-\log \varepsilon\left(\overline{\mathscr{H}_{0}^{M}}\right)+\frac{1}{2} \log \operatorname{rg}_{\mathbb{Z}}\left(\mathscr{H}_{0}^{M}\right)+\frac{\log \left|\Delta_{K}\right|}{2[K: \mathbb{Q}]} .
$$

On majore le terme $-\log \varepsilon\left(\overline{\mathscr{H}_{0}^{M}}\right)$ à l'aide de la théorie de l'intersection arithmétique. On commence par appliquer [Bost et al. 1994, proposition 3.2.1]; comme $L$ est ample avec $c_{1}(L)$ définie positive (par [Bost et al. 1994, proposition 3.2.4]), pour une section $s \in H^{0}\left(\mathscr{L}, \mathscr{L}^{M}\right)$, on a :

$$
h_{L}(\operatorname{div}(s))=M h_{L}(X)+\int_{X(\mathbb{C})} \log \|s\| c_{1}(L)^{d} \geq 0 .
$$

De plus, comme $c_{1}(L)$ est définie positive, on a :

$$
\int_{X(\mathbb{C})} \log \|s\| c_{1}(L)^{d} \leq \max _{\sigma \in \Sigma_{\infty}} \log \|s\|_{\sup , \sigma} \int_{X(\mathbb{C})} c_{1}(L)^{d} .
$$


Par le théorème de Wirtinger (voir [Griffiths et Harris 1978, page 171]), la dernière intégrale est $\operatorname{deg}_{L}(X)$. On a donc :

$$
-\max _{\sigma \in \Sigma_{\infty}} \log \|s\|_{\text {sup }, \sigma} \leq M \frac{h_{L}(X)}{\operatorname{deg}_{L}(X)} .
$$

Soit $\sigma_{0}$ tel que $\|s\|_{\text {sup }, \sigma_{0}}$ réalise le maximum sur toutes les places archimédiennes. Par choix de la norme de Löwner associée à la norme du sup sur $\mathscr{H}_{0}^{M}$, on a :

et par suite :

$$
\max _{\sigma \in \Sigma_{\infty}}\|s\|_{\text {sup }, \sigma} \leq \sqrt{2 \operatorname{rg} \mathscr{H}_{0}^{M}}\|s\|_{L, \sigma_{0}}
$$

$$
\begin{aligned}
-\log \|s\| & =-\frac{1}{2} \log \sum_{\sigma \in \Sigma_{\infty}}\|s\|_{L, \sigma}^{2} \leq-\log \|s\|_{L, \sigma_{0}} \\
& \leq-\max _{\sigma \in \Sigma_{\infty}} \log \|s\|_{\text {sup }, \sigma}+\frac{1}{2} \log \left(2 \operatorname{rg} \mathcal{H}_{0}^{M}\right) .
\end{aligned}
$$

On en déduit que :

$$
-\log \varepsilon\left(\overline{\mathscr{H}_{0}^{M}}\right) \leq M \frac{h_{\bar{L}}(X)}{\operatorname{deg}_{L}(X)}+\frac{1}{2} \log \left(2 \operatorname{rg} \mathscr{H}_{0}^{M}\right) .
$$

Il reste à majorer le rang de $\mathscr{H}_{0}^{M}$. Le fibré $L$ définit un plongement projectivement normal de $A$, et les éléments de $\mathscr{H}_{0}^{M}$ sont des restrictions à $X$ de sections globales sur $A$, donc on est en mesure d'appliquer le théorème principal de [Chardin 1989] :

$$
\operatorname{rg}_{\mathbb{Z}} \mathscr{H}_{0}^{M} \leq[K: \mathbb{Q}] \operatorname{rg} \mathscr{H}_{0}^{M} \leq[K: \mathbb{Q}](M+d)^{d} \operatorname{deg}_{L}(X) .
$$

La proposition est entièrement démontrée.

On peut maintenant majorer la pente maximale du fibré des sections avec multiplicité. Le fibré $\mathscr{W}^{\vee}$ étant constant, on a l'isomorphisme (isométrique) :

$$
\mathscr{H}_{m}^{M} \simeq \mathscr{H}_{0}^{M} \otimes S^{m} W^{\vee} \text {. }
$$

Bost a conjecturé que la pente maximale du produit tensoriel est la somme des pentes maximales. Les meilleurs résultats connus dans cette direction sont ceux de Bost et Künnemann [2007], et de Chen [2006], qui a démontré dans sa thèse :

Théorème 3.13. Soient $\overline{\mathscr{E}}_{1}, \ldots, \overline{\mathscr{E}}_{n}$ des fibrés hermitiens non-nuls sur $\operatorname{Spec} \mathscr{O}_{K}$. Alors :

$$
\left.\hat{\mu}_{\max }\left(\overline{\mathscr{E}}_{1} \otimes \cdots \otimes \overline{\mathscr{E}}_{n}\right) \leq \sum_{i=1}^{n}\left(\hat{\mu}_{\max }\left(\overline{\mathscr{E}}_{i}\right)+\log \operatorname{rg} \mathscr{E}_{i}\right)\right) .
$$

Rappelons que par $c(\mathcal{W})$, on désigne une borne $\geq 1$ pour la hauteur d'une base entière de $\mathscr{W}$. On peut maintenant démontrer le corollaire suivant :

Corollaire 3.14. On a la majoration, pour la pente maximale:

$$
\hat{\mu}_{\max }\left(\overline{\mathscr{H}_{m}^{M}}\right) \leq M \frac{h_{\bar{L}}(X)}{\operatorname{deg}_{L}(X)}+2 d \log (M+d)+2 \log \operatorname{deg}_{L}(X)+c_{9} m c(\mathcal{W}),
$$

où $c_{9}$ ne dépend que de $(A, L)$. 
Démonstration. Le théorème précédent nous montre que :

$$
\hat{\mu}_{\max }\left(\overline{\mathscr{H}_{m}^{M}}\right) \leq \hat{\mu}_{\max }\left(\overline{\mathcal{H}_{0}^{M}}\right)+\hat{\mu}_{\max }\left(S^{m}\left(\overline{W^{\vee}}\right)\right)+\log \left(\operatorname{rg} \mathscr{H}_{0}^{M}\right)+\log \left(\operatorname{rg} S^{m}\left(\mathcal{W}^{\vee}\right)\right) .
$$

Les termes relatifs à $\overline{\mathscr{H}_{0}^{M}}$ ont été calculés dans la proposition précédente. Le rang de $S^{m}\left(W^{\vee}\right)$ est donné par la formule classique :

$$
\operatorname{rg} S^{m}\left(W^{\vee}\right)=\left(\begin{array}{c}
m+g-1 \\
g-1
\end{array}\right) \leq(m+g)^{g-1},
$$

et sa pente maximale a été majorée en 3B1.

\section{Choix des fibrés et du morphisme}

On rappelle que $A$ est une variété abélienne définie sur un corps de nombres $K$, munie d'un fibré $L$ ample et symétrique, qu'on pourra supposer très ample quitte à considérer $L^{\otimes 3}$. Quitte à prendre une extension finie de $K$ (ne dépendant que de $A$ ), on prend un modèle de Moret-Bailly $(\mathscr{A}, \mathscr{L}, 0)$ de $(A, L)$, suivant la terminologie de [Bost 1996a]. Ce modèle est en particulier semi-abélien [Gaudron 2006, définition-théorème 4.3]. On suppose de plus que $\mathscr{A}$ vérifie l'hypothèse $H$. On vérifie aisément que celle-ci implique $\mathrm{H}^{\prime}$ et on prend $\alpha=g$ dans la définition de $\mathscr{P}_{A}$.

On prend une sous-variété stricte $V$ de $A$, de codimension $r$, qui n'est pas incluse dans le translaté d'une sous-variété abélienne stricte. On pose aussi $X=V+H_{0}$, où $H_{0}$ est un certain sous-groupe fini de $A$. On va construire un fibré hermitien $\overline{\mathscr{E}}_{M}$ associé à un espace vectoriel $E$, une suite de fibrés $\overline{\mathscr{G}}_{k}, k \in I$ (pour un certain ensemble fini $I$ ), correspondant à une fibration d'un espace vectoriel $F$, et un morphisme $\phi$ de restriction entre $E$ et $F$. Les $\overline{\mathscr{G}}_{k}$ seront définis à partir de l'espace des sections d'une puissance de $\mathscr{L}$ sur des modèles entiers de $X$ et de ses translatés par des points de torsion bien choisis. La partie précédente nous permettra de calculer les termes de pentes associés à ces fibrés. À la fin de cette partie, on fixera les paramètres et on supposera par l'absurde que le minimum essentiel de $V$ est majoré en fonction des paramètres.

4A. Le plongement étiré. Pour éliminer la constante de comparaison entre hauteur projective et hauteur de Néron-Tate sur $A$, on considère classiquement un plongement étiré. Soit $M$ un entier supérieur ou égal à 1 . La multiplication par $M$ sur $A$ est notée $[M]$ et on définit :

$$
\psi_{M}: A \rightarrow A \times A, \quad x \mapsto(x,[M] x) .
$$

Ce plongement a été utilisé pour la première fois par Laurent [1983] pour étudier le problème de Lehmer elliptique. Son principe est le suivant : les techniques 
diophantiennes nous renseignent sur la hauteur projective, et le minimum essentiel fait intervenir la hauteur de Néron-Tate associée au plongement. On sait que la différence entre ces deux hauteurs est bornée mais la hauteur de Néron-Tate peut être très petite; il y a donc une perte d'information sur la hauteur projective. Le plongement étiré multiplie la hauteur par un paramètre assez grand, qui rend négligeable la constante de comparaison.

Soit $L_{M}:=\psi_{M}^{*}\left(\pi_{1}^{*} L \otimes \pi_{2}^{*} L\right) \simeq L^{\otimes\left(M^{2}+1\right)}$ et $\mathscr{L}_{M}:=\psi_{M}^{*}\left(\pi_{1}^{*} \mathscr{L} \otimes \pi_{2}^{*} \mathscr{L}\right)$. On note $\operatorname{deg}_{M}$ le degré, et $\hat{h}_{M}$ la hauteur canonique, par rapport au fibré $L_{M}$. Le lemme suivant indique la variation de la hauteur et du degré par changement de fibré :

Lemme 4.1. Si V est une sous-variété de A, on a la variation suivante de la hauteur canonique:

$$
\hat{h}_{M}(V)=\left(M^{2}+1\right)^{\operatorname{dim} V+1} \hat{h}_{L}(V) ;
$$

et la formule suivante pour le degré:

$$
\operatorname{deg}_{M}(V)=\left(M^{2}+1\right)^{\operatorname{dim} V} \operatorname{deg}(V) .
$$

Démonstration. Ces formules sont démontrées, par exemple, dans [Philippon 1995, proposition 7].

Dans cette partie et la suivante, on travaillera donc dans le plongement étiré et on notera $A_{M}$ l'image de $A$ par le plongement $\psi_{M}$.

4B. Le fibré de départ. Commençons par décrire le premier fibré. On pose $\mathscr{E}_{M}:=$ $H^{0}\left(\mathscr{A}, \mathscr{L}_{M}\right)$. On note aussi $E$ le tensorisé de $\mathscr{\mathscr { C }}_{M}$ avec $K$. On a vu en $3 \mathrm{~B} 2$ comment munir ce fibré d'une structure hermitienne et on a donné son degré. On fait une hypothèse qui sera aisément vérifiée par la suite :

$$
\log M^{2}>2\left(\frac{2}{g} h_{F}(A)+\log (2 \pi g !)\right) .
$$

Lemme 4.2. On a la minoration suivante pour le degré normalisé de $\overline{\mathscr{E}_{M}}$ :

$$
\widehat{\operatorname{deg}} \overline{\mathscr{E}} M \geq c_{10}\left(M^{2}\right)^{g} \log M,
$$

pour une constante $c_{10}>0$ ne dépendant que de A.

Démonstration. En multipliant la pente par le rang, la proposition 3.11 donne :

$$
\widehat{\operatorname{deg}} \overline{\mathscr{E}_{M}}=\left(M^{2}+1\right)^{g} \frac{\operatorname{deg}_{L}(A)}{g !}\left(-\frac{1}{2} h_{F}(A)+\frac{1}{4} \log \frac{\left(M^{2}+1\right)^{g} \operatorname{deg}_{L}(A)}{(2 \pi)^{g} g !}\right) .
$$

Notons que la hauteur de Faltings ne dépend que de la classe d'isomorphisme de $A$. L'hypothèse faite sur les paramètres donne immédiatement le lemme, avec une constante $c_{10}>0$ ne dépendant que de $A$ et facilement explicitable. 
4C. Fibration de l'espace d'arrivée. On introduit maintenant la notion de voisinage infinitésimal [Bost 1996a], qui sera défini à partir d'un fermé $X$ de $A$ et d'un ordre de dérivation $l$. On commence par choisir la base du tangent $\left(e_{1}, \ldots, e_{g}\right)$ sur $A$ correspondant à la base de dérivations algébriques fixée en 2C. L'action de l'isogénie $[M]$ sur le tangent étant la multiplication par $M$, on en déduit d'abord une base $\left(e_{1}, \ldots, e_{2 g}\right)$ de $t_{A^{2}}$, puis on pose $f_{i}=e_{i}+M e_{g+i}$. La famille $\left(f_{1}, \ldots, f_{g}\right)$ est une base de l'espace tangent $t_{A_{M}}$, image de $t_{A}$ par l'étirement (voir 3B1) et on note (par abus de langage) $\left(\partial_{1}, \ldots, \partial_{g}\right)$ la base de dérivations associée. Si $x \in A$, on en déduit par translation une base de dérivations $\left(\partial_{1, x}, \ldots, \partial_{g, x}\right)$ en $x$ (dans le plongement étiré). On notera aussi par la suite :

$$
\partial_{x}^{\lambda}=\frac{1}{\prod_{1=i}^{g} \lambda_{i} !} \partial_{1, x}^{\lambda_{1}} \cdots \partial_{g, x}^{\lambda_{g}} .
$$

Soit $X$ un fermé de Zariski de $A$. On définit le schéma $V\left(X, t_{A_{M}}, l\right)$ de la façon suivante :

- si $l=0, V\left(X, t_{A_{M}}, l\right)$ est le sous-schéma réduit de $A$ défini par $X$;

- si $l=1$ et $X=0, V\left(0, t_{A_{M}}, 1\right)$ est le voisinage infinitésimal d'ordre 1 de 0 ;

- si $l \geq 1, V\left(X, t_{A_{M}}, l\right)$ est l'image dans $A$ du schéma $X \times V\left(0, t_{A_{M}}, 1\right)^{l}$ par le morphisme d'addition $A^{l+1} \rightarrow A$.

Le schéma $V\left(X, t_{A_{M}}, l\right)$ admet pour support le fermé $X$ et son faisceau d'idéaux $\mathscr{I}$ est défini par :

$$
s \in \mathscr{I} \Longleftrightarrow \partial_{x}^{\lambda} s=0 \text { pour tout } x \in X \text { et tout } \lambda \in \mathbb{N}^{g} \text { tel que } \sum_{i=1}^{g} \lambda_{i} \leq l .
$$

L'espace vectoriel d'arrivée sera formé de sous-espaces de sections sur $X$ et de nombreux translatés de $X$ par des points de torsion, avec multiplicité. On précise d'abord les points de torsion en lesquels on extrapole, et la multiplicité, puis on met un ordre sur cet ensemble. On se donne $T_{0}>0$. Pour $1 \leq n \leq r$ ( $r$ étant la codimension de $V$ et de $X$ ), on se donne aussi deux nombres positifs $T_{n}$ (qui correspond à la multiplicité après $n$ extrapolations), et $N_{n}$ (qui borne les normes des premiers d'extrapolation). Puis on définit les ensembles $\mathscr{P}_{n}$ :

$$
\mathscr{P}_{n}=\left\{\mathfrak{p} \in \mathscr{P}_{A}: \mathbf{N}(\mathfrak{p}) \in\left[N_{n} / 2 ; N_{n}\right]\right\} .
$$

On note $\mathscr{P}$ l'ensemble des premiers de $\mathbb{Z}$. La projection des $\mathscr{P}_{n}$ sur $\mathbb{Z}$ est donnée par :

$$
\mathscr{P}_{n, \mathbb{Z}}=\left\{p \in \mathscr{P}: \exists \mathfrak{p} \in \mathscr{P}_{n}, \mathfrak{p} \mid p\right\} .
$$

Le choix des paramètres sera tel que les ensembles $\mathscr{P}_{n}$ (resp. $\mathscr{P}_{n, \mathbb{Z}}$ ) soient disjoints. On note :

$$
\operatorname{Tor}_{A, n}=\left\{\begin{array}{l}
P \in A[p]: \exists \mathfrak{p} \in \mathscr{P}_{n} \text { divisant } p, \text { tel que pour tout } \\
\mathfrak{q} \in \mathcal{O}_{K(P)} \text { divisant } \mathfrak{p}, P \text { se réduit sur } 0 \text { modulo } \mathfrak{q}
\end{array}\right\} .
$$


On note pour toute la suite $K^{\prime}$ le corps engendré par $K$, par un corps de définition de $V$ et par les coordonnées projectives des points de la réunion $\bigcup_{1 \leq n \leq r} \operatorname{Tor}_{A, n}$. Les fibrés et les morphismes qu'on va considérer seront tous définis sur $K^{\prime}$; en fait, comme on considère des $\hat{O}_{K}$-fibrés, les calculs de pentes valables sur $\hat{O}_{K}$ se transportent sans changer à $\hat{O}_{K^{\prime}}$, et le corps $K^{\prime}$ intervient uniquement dans les estimations ultramétriques.

On ordonne les points de $\operatorname{Tor}_{A, n}$ en les classant d'abord selon le plus petit premier $p$ de torsion (pour l'ordre naturel sur $\mathbb{Z}$ ) puis en choisissant arbitrairement un ordre à $p$ fixé. Pour $i=\left(i_{1}, \ldots, i_{r}\right)$, où $i_{n}$, pour $1 \leq n \leq r$, est un indice dans [1, $\left.\left|\operatorname{Tor}_{A, n}\right|\right]$, on note $P_{i}$ le point $P_{i_{1}}+\cdots+P_{i_{r}}$. On note $I$ l'ensemble de ces multiindices, qu'on ordonne avec l'ordre lexicographique. On confondra dans la suite l'ensemble $I$ et son image dans $\mathbb{N}$ par l'indexation, et les éléments $i \in I$ pourront être vus comme des entiers via cette identification. On construit, pour $i \in I$, une suite de fermés $X_{i}=X+P_{i}$. On pose enfin, pour $i \in I: T_{(i)}=T_{n_{i}}$ et $N_{(i)}=N_{n_{i}}$, où $n_{i}$ est le plus grand des $j$ tels que $P_{i_{j}} \neq 0$.

On définit maintenant :

$$
S:=\bigcup_{i \in I} V\left(X_{i}, t_{A_{M}}, T_{(i)}\right) \quad \text { et } \quad F:=H^{0}\left(A, L_{M}\right)_{\mid S} .
$$

On note $\phi$ le morphisme (surjectif) de restriction de $E$ vers $F$, et pour $k \in I$ un entier, on pose :

$$
S_{k}=\bigcup_{i \leq k} V\left(X_{i}, t_{A_{M}}, T_{(i)}\right) .
$$

Ceci permet de définir $F_{k}$, le noyau de la restriction :

$$
H^{0}\left(A, L_{M}\right)_{\mid S} \rightarrow H^{0}\left(A, L_{M}\right)_{\mid S_{k}} .
$$

La suite décroissante des $F_{k}$ est une filtration de $F$ et on en déduit une filtration de $E$ en posant $E_{k}:=\phi^{-1}\left(F_{k-1}\right)$. Soit aussi $G_{k}=F_{k-1} / F_{k}$ et $\phi_{k}: E_{k} \rightarrow G_{k}$ l'application linéaire déduite de $\phi$ et de la projection canonique : $F_{k-1} \rightarrow G_{k}$. En appliquant le lemme des serpents aux deux suites exactes :

$$
\begin{gathered}
0 \rightarrow F_{k} \rightarrow H^{0}\left(A, L_{M}\right)_{\mid S} \rightarrow H^{0}\left(A, L_{M}\right)_{\mid S_{k}} \rightarrow 0, \\
0 \rightarrow F_{k-1} \rightarrow H^{0}\left(A, L_{M}\right)_{\mid S} \rightarrow H^{0}\left(A, L_{M}\right)_{\mid S_{k-1}} \rightarrow 0,
\end{gathered}
$$

on voit que $G_{k}$ s'identifie au noyau de :

$$
H^{0}\left(A, L_{M}\right)_{\mid S_{k}} \rightarrow H^{0}\left(A, L_{M}\right)_{\mid S_{k-1}} .
$$

qui est un sous-espace vectoriel de :

$$
H_{k}:=H^{0}\left(A, \operatorname{Sym}^{T_{(k)}}\left(t_{A_{M}}^{\vee}\right) \otimes L_{M}\right)_{\mid X_{k}} .
$$


Remarque. On a choisi ici de noter Sym le fibré symétrique, pour éviter toute confusion avec le schéma $S$.

On se donne un modèle entier avec structure hermitienne $\overline{\mathscr{H}}_{k}$ de $H_{k}$ comme dans 3B3 et on en déduit un modèle entier avec structure hermitienne $\bar{\varphi}_{k}$ de $G_{k}$.

Lemme 4.3. Il existe une constante $c_{11}$ ne dépendant que de A telle que :

$$
\hat{\mu}_{\max }\left(\overline{\mathscr{G}}_{k}\right) \leq c_{11}\left(M^{2} \hat{\mu}_{L}^{\mathrm{ess}}(X)+\log \operatorname{deg}_{L}(X)+T_{(k)} \log \left(M+T_{(k)}\right)\right) .
$$

Démonstration. On utilise le corollaire 3.14 pour majorer la pente maximale de $\overline{\mathscr{H}}_{k}$. En notant $h_{M}$ la hauteur projective associée à $L_{M}$, on commence par remarquer qu'il existe une constante $c_{11}$ ne dépendant que de $A$ telle que :

$$
\frac{h_{M}\left(X_{k}\right)}{\operatorname{deg}_{M}\left(X_{k}\right)} \leq \frac{\hat{h}_{M}\left(X_{k}\right)}{\operatorname{deg}_{M}\left(X_{k}\right)}+c_{11} \leq\left(M^{2}+1\right) \frac{\hat{h}_{L}\left(X_{k}\right)}{\operatorname{deg}_{L}\left(X_{k}\right)}+c_{11},
$$

en comparant la hauteur projective et la hauteur canonique dans le plongement étiré [David et Philippon 2002, propositions 3.9 et 3.14], puis grâce au lemme 4.1. L'inégalité des minima successifs (démontrée dans [Zhang 1995b, Theorem 5.2]) donne donc, comme $M \geq 1$ :

$$
\frac{h_{M}\left(X_{k}\right)}{\operatorname{deg}_{M}\left(X_{k}\right)} \leq 2 g M^{2} \hat{\mu}_{L}^{\mathrm{ess}}(X)+c_{11} .
$$

Le minimum essentiel, tout comme le degré, n'est pas modifié lorsqu'on translate par des points d'ordre fini. Par choix de la base de dérivation sur $\mathscr{A}_{M}$, en tenant compte de l'action de $[M]$ sur le tangent et en bornant la hauteur de la base de dérivations algébriques par une constante ne dépendant que de $A$, on peut choisir :

$$
c\left(t_{\mathscr{A}_{M}}\right) \leq \log M+c_{11} .
$$

Comme $\overline{\mathscr{G}}_{k}$ s'injecte isométrique dans $\overline{\mathscr{H}}_{k}$, on a :

$$
\hat{\mu}_{\max }\left(\overline{\mathscr{G}}_{k}\right) \leq \hat{\mu}_{\max }\left(\overline{\mathscr{H}}_{k}\right) \leq c_{11}\left(M^{2} \hat{\mu}_{L}^{\mathrm{ess}}(X)+\log \operatorname{deg}_{L}(X)+T_{(k)} \log \left(M+T_{(k)}\right)\right),
$$

quitte à prendre $c_{11}$ assez grande en fonction de $A$.

4D. Choix des paramètres et hypothèse sur le minimum essentiel. Ce choix doit permettre d'assurer l'injectivité du morphisme $\phi$ et de contredire l'inégalité des pentes. On suit la stratégie suivante :

- On définit les paramètres $M, T_{0}, N_{1}$ de telle manière que le terme (correspondant au fermé $X$ non-translaté) :

$$
\operatorname{rg} \mathscr{G}_{0}\left(\hat{\mu}_{\max }\left(\overline{\mathscr{G}_{0}}\right)+h\left(\phi_{0}\right)\right)
$$

soit inférieur à $\widehat{\operatorname{deg}}(\overline{\mathscr{E}} M)$. 
- Les relations entre les paramètres $T_{i}$ et $N_{i}$ sont telles que les contributions des termes suivants dans l'inégalité des pentes soient négatives.

- Le paramètre d'étirement $M$ est pris aussi petit que possible, ce qui permet de montrer que le morphisme $\phi$ est injectif; il est aussi choisi de telle sorte que $M^{2} \hat{\mu}_{L}^{\text {ess }}(X)$ soit majoré par une constante, ce qui détermine la minoration obtenue pour le minimum essentiel.

On commence par introduire l'indice d'obstruction avec poids $\omega_{L}(x, X)$; celuici permet classiquement de prendre en compte la hauteur des dérivées dans un lemme de Siegel. Il aura un emploi similaire dans le cadre de la théorie des pentes.

Définition 4.4. Soit $X$ un fermé de Zariski strict de $A$ et $x$ un réel positif. On pose :

$$
\omega_{L}(x, X)=\inf \left(x \operatorname{deg}_{L}(Z)\right)^{1 / \operatorname{codim} Z},
$$

où l'infimum porte sur l'ensemble des fermés de Zariski (équidimensionnels) stricts de $A$ contenant $X$.

On utilisera souvent le lemme suivant, qui compare l'indice d'obstruction simple, ne prenant en compte que les hypersurfaces, et l'indice d'obstruction avec poids :

Lemme 4.5. Soit $X$ un fermé strict (équidimensionnel) de A en codimension $r$. Il existe une constante $c_{12}$ ne dépendant que de A telle que pour tout réel $x \geq 1$ :

$$
c_{12} x^{1 / r} \omega_{L}(X) \leq \omega_{L}(x, X) \leq x \omega_{L}(X) .
$$

Démonstration. L'inégalité de droite est immédiate et celle de gauche est une conséquence de [Chardin 1990, corollaire 2 et exemple 1]. Remarquons qu'un fibré très ample étant fixé, seule la dimension du projectif dans lequel on plonge $A$ intervient dans $c_{12}$.

On se donne une constante $C_{0}$ ne dépendant que de $A$, grande devant toutes les constantes du problème, dans un sens explicitable au cours de la preuve. Soit $\Delta$ le paramètre :

$$
\Delta=C_{0}^{2} \log \left(3 \operatorname{deg}_{L}(V)\right) .
$$

C'est à partir de ce paramètre, qui est de l'ordre de $\log \operatorname{deg}_{L}(V)$, qu'on va définir tous les autres paramètres. Son avantage — comparé à $\log \left(\operatorname{deg}_{L}(V)\right)$ - est d'être inconditionnellement grand devant les constantes intervenant au cours de la preuve, par choix de $C_{0}$.

En vue de la descente, nous introduisons deux paramètres qui permettront d'itérer la construction. Soient donc $R$ et $\rho$ deux entiers strictement positifs tels que :

$$
\Delta \geq \log R \text { et } \rho \leq\left(9(2 r)^{r+1}\right)^{r-1} .
$$

Dans le formalisme des pentes, on regarde la restriction d'une section aux translatés de $V$ par $n$ points de torsion, où $0 \leq n \leq r$. On prend un paramètre $T_{0}$ correspondant à la multiplicité initiale et on associe à tout $n \in[1 ; r]$ des paramètres 
spécifiques, à savoir une multiplicité $T_{n}$, et une borne $N_{n}$ pour la norme des premiers de torsion, ce qui détermine un ensemble de premiers $\mathscr{P}_{n}$. Le lien entre ces paramètres est choisi de telle sorte que la contribution des termes de pente, pour des indices strictement positifs, soient négatifs. On prend d'abord :

$$
N_{n}=\Delta^{\rho(2 r)^{r+2-n}} .
$$

Chaque $N_{n}$ est donc négligeable devant le précédent ; les raisons de ce choix seront plus claires au cours de la preuve de la proposition 6.5, qui montrera quasiment l'injectivité du morphisme $\phi$. Passons à la multiplicité. Par récurrence descendante, pour $0 \leq n \leq r-1$, on pose :

$$
T_{n}=T_{n+1}\left(N_{n+1} \Delta^{2}\right)
$$

Puis on pose :

$$
T_{r}=1 \text {. }
$$

Ces formules déterminent complètement $T_{0}$ par itération :

$$
T_{0}=\Delta^{2 r} N_{1} \cdots N_{r} .
$$

On finit par le paramètre $M$. Le but est de prendre $M^{2}$ assez grand pour que le premier terme dans la somme de l'inégalité de pentes soit plus petit que le degré de $\overline{\mathscr{E}} M$. Cette condition s'apparente à celle qu'on obtiendrait par un lemme de Siegel dans une preuve classique de transcendance. Pour montrer l'injectivité de $\phi$, on aura au contraire besoin que $M^{2}$ ne soit pas trop grand. On choisit donc :

$$
M=\left\lfloor\left(T_{0} \omega_{L}\left(\Delta T_{0}, X\right)\right)^{1 / 2}\right\rfloor+1 .
$$

On rappelle que $V$ est une sous-variété stricte de $A$ qui n'est pas incluse dans un translaté de sous-variété abélienne stricte, et que : $X=V+H_{0}$, où $H_{0}$ est un sous-groupe fini de $A$. On suppose que le cardinal de $H_{0}$ n'est pas trop gros :

$$
\log \left|H_{0}\right| \leq \Delta \text {. }
$$

Puis on fait l'hypothèse suivante sur le minimum essentiel de $X$ :

$$
\hat{\mu}_{L}^{\mathrm{ess}}(X)<\frac{\Delta}{T_{0} \omega_{L}\left(\Delta T_{0}, X\right)} .
$$

\section{Utilisation de l'inégalité des pentes}

On a déjà calculé le degré de $\overline{\mathscr{E}}_{M}$ et les pentes maximales. On veut maintenant majorer le rang des $\mathscr{G}_{k}$ et la hauteur des $\phi_{k}$. Les estimations ultramétriques dans la hauteur des $\phi_{k}$ sont le point crucial de la preuve et ont été préparées par la partie 2. Les estimations archimédiennes, enfin, utilisent essentiellement l'inégalité de Cauchy (en plusieurs variables). 


\section{A. Majoration du rang.}

5A1. Nombre de dérivations. Le calcul du nombre de dérivations est classique dans les preuves de transcendance. Si $X$ est un fermé équidimensionnel plongé par un fibré très ample $L$ dans un espace projectif $\mathbb{P}^{n}$ sur $\overline{\mathbb{Q}}$, associé à un idéal homogène $I$, et $m$ un entier, on pose

$$
H\left(X, L^{\otimes m}\right):=\operatorname{dim}\left[\overline{\mathbb{Q}}\left[X_{0}, \ldots, X_{n}\right] / I\right]_{m},
$$

où on se restreint à la composante homogène de degré $m$ du quotient. La prise en compte de l'indice d'obstruction avec poids nous amène à faire le raisonnement qui suit avec un fermé équidimensionnel $Z_{k}$ contenant $X_{k}$, de codimension $r^{\prime} \leq r=$ $\operatorname{codim} X_{k}$.

Proposition 5.1. On a l'inégalité suivante:

$$
\operatorname{rg} \mathscr{G}_{k} \leq \operatorname{rg} \mathscr{H}_{k} \leq T_{(k)}^{r^{\prime}} H\left(Z_{k}, L_{M}\right) .
$$

Démonstration. La première inégalité est claire. On remarque ensuite que l'inclusion $X_{k} \subset Z_{k}$ permet de se ramener à la majoration du rang du fibré $\mathscr{H}_{k}$, dans lequel on a remplacé $X_{k}$ par $Z_{k}$. Le plongement de $A$ par $L_{M}$ étant projectivement normal, et les sections considérées étant des restrictions de sections globales sur $A$, elles appartiennent au quotient $\overline{\mathbb{Q}}\left[X_{0}, \ldots, X_{n}\right] / I\left(Z_{k}\right)$. On applique alors l' $\ll$ astuce de Philippon-Waldschmidt $\gg$ (voir [Amoroso et David 2003, lemme 2.5], ou encore [David et Hindry 2000, 5.3]), qui donne :

$$
\begin{aligned}
\operatorname{dim} H^{0}\left(A, \operatorname{Sym}^{T_{(k)}}\left(t_{A_{M}}^{\vee}\right) \otimes L_{M}\right)_{\mid Z_{k}} & \leq\left(\begin{array}{c}
T_{(k)}+r^{\prime}-1 \\
r^{\prime}-1
\end{array}\right) H\left(Z_{k}, L_{M}\right) \\
& \leq T_{(k)}^{r^{\prime}} H\left(Z_{k}, L_{M}\right) .
\end{aligned}
$$

5A2. Le théorème de Chardin. Pour majorer $H\left(Z_{k}, L_{M}\right)$, on ne peut pas se contenter du théorème de Hilbert-Samuel géométrique. Celui-ci donne une estimation asymptotique, ce qui oblige à introduire une constante indéterminée dépendant de $Z_{k}$ (et par suite de l'indice d'obstruction).

Proposition 5.2. On $a$ :

$$
H\left(Z_{k}, L_{M}\right) \leq\left(\begin{array}{c}
1+g-r^{\prime} \\
g-r^{\prime}
\end{array}\right) \operatorname{deg}_{M}\left(Z_{k}\right)
$$

Démonstration. C'est une conséquence du résultat principal de [Chardin 1989], valable pour un fermé équidimensionnel.

On regroupe les deux derniers résultats dans le corollaire suivant :

Corollaire 5.3.

$$
\operatorname{rg} \mathscr{G}_{k} \leq \frac{g\left(2 M^{2}\right)^{g}}{\Delta T_{0}}
$$


Démonstration. On combine les deux dernières propositions en remarquant que le coefficient binômial apparaissant dans la dernière est inférieur ou égal à $g$. Puis on obtient, grâce au lemme 4.1 :

$$
\operatorname{rg} \mathscr{G}_{k} \leq g T_{(k)}^{r^{\prime}} \operatorname{deg}_{M}\left(Z_{k}\right) \leq g T_{(k)}^{r^{\prime}}\left(2 M^{2}\right)^{g-r^{\prime}} \operatorname{deg}_{L}\left(Z_{k}\right)
$$

Ceci est vrai pour toute variété $Z_{k}$ contenant $X_{k}$. Soit donc $Z_{k}$ une sous-variété, de codimension $r^{\prime}$, telle que (on se ramène à $X$ car le degré est invariant par translation) :

$$
\omega_{L}\left(\Delta T_{0}, X\right)=\left(\Delta T_{0} \operatorname{deg}_{L}\left(Z_{k}\right)\right)^{1 / r^{\prime}} .
$$

On a donc, par choix du paramètre $M$ :

$$
\operatorname{rg} \mathscr{G}_{k} \leq \frac{g\left(2 M^{2}\right)^{g}}{\Delta T_{0}}\left(\frac{T_{(k)}}{T_{0}} \frac{\omega_{L}\left(\Delta T_{0}, X\right)}{\omega_{L}\left(\Delta T_{0}, X\right)}\right)^{r^{\prime}},
$$

et le résultat suit puisque $T_{(k)} \leq T_{0}$.

5B. Normes ultramétriques des morphismes. Soit $k \in I$ un entier. Si $\mathfrak{q}$ est un idéal premier de $0_{K^{\prime}}$, la norme q-adique de $\phi_{k}$ est agréablement majorée si la $k$ ème étape dans la filtration correspond à la translation par certains points de torsion de $A$.

Proposition 5.4. Si $\mathfrak{p}:=\mathfrak{q} \cap \mathfrak{O}_{K} \in \bigcup_{1 \leq n \leq r} \mathscr{P}_{n}$, on $a$ :

$$
\log \left\|\phi_{k}\right\|_{\mathfrak{q}} \leq 0 \text {. }
$$

Si de plus, en notant $P_{k}=P_{k_{1}}+\cdots+P_{k_{n}}$ le point associé à $k$, le point $P_{k_{n}}$ est un point de p-torsion (où $(p)=\mathfrak{p} \cap \mathbb{Z})$ se réduisant sur $0 \bmod \mathfrak{q}$, on $a$ :

$$
\log \left\|\phi_{k}\right\|_{\mathfrak{q}} \leq-n_{\mathfrak{q}}\left(T_{n-1}-T_{n}\right) \frac{\log p}{p} .
$$

Démonstration. Commençons par l'inégalité faible. Soit $s$ un élément de $E_{k}$ tel que $\|s\|_{\mathfrak{q}} \leq 1$; soit $\mathscr{X}_{k}$ le modèle entier de $X_{k}$ choisi dans la définition de $\mathscr{G}_{k}$, et $x$ un élément de $\mathscr{X}_{k}$. Soit aussi $\lambda \in \mathbb{N}^{g}$ tel que $\sum_{1 \leq i \leq g} \lambda_{i} \leq T_{(k)}$. On a :

$$
\partial_{x}^{\lambda} s=\partial^{\lambda}\left(s \circ \tau_{x}\right)
$$

Par définition de $\mathscr{P}_{A}$, les coefficients de $\tau_{x}$ sont $\mathfrak{p}$-entiers (voir (2-1)). De plus, le choix de $\mathscr{P}_{A}$ permet aussi que l'opérateur différentiel $\partial^{\lambda}$ appliqué en chaque monôme affine s'exprime comme un polynôme en les coordonnées affines à coefficients p-entiers (par (2-2)). En remarquant que la base de dérivations sur $A$ est algébrique (ce qui fait disparaître les factorielles au dénominateur quand on dérive des polynômes) et que la base de dérivations sur $A_{M}$ est obtenue par combinaisons linéaires à coefficients entiers des dérivations sur $A$, on a :

$$
\left|\partial_{x}^{\lambda} s\right|_{\mathfrak{q}} \leq 1
$$


Ceci étant vrai pour tout $x \in \mathscr{X}_{k}$ et tout $\lambda \in \mathbb{N}^{g}$ tel que $\sum \lambda_{i} \leq T_{(k)}$, on en déduit :

$$
\left\|\phi_{k}(s)\right\|_{\mathfrak{q}} \leq 1 .
$$

La première partie de la proposition est donc démontrée en passant au sup sur $s \in E_{k}$.

Pour l'inégalité forte, on écrit $P_{k}=P_{k^{\prime}}+P_{k_{n}}$ le point de torsion associé à $k$, où $P_{k_{n}}$ se réduit sur 0 modulo q. Pour $x \in \mathscr{X}_{k}$, on note $x=x^{\prime}+P_{k_{n}}$. Soit $\mathbf{t}_{v}=\left(t_{1, v}, \ldots, t_{g, v}\right)$ une base de paramètres bien définie en $P_{k_{n}}$, suivant le lemme 2.10. On reprend les notations convenues avant la proposition 2.11. L'isomorphisme :

$$
\hat{A}_{\mathfrak{O}_{\mathfrak{p}}} \simeq \mathcal{O}_{\mathfrak{p}} \llbracket t_{1, v}, \ldots, t_{g, v} \rrbracket
$$

induit une application :

$$
H^{0}\left(\mathscr{A}_{\mathfrak{O}_{\mathfrak{p}}}, \mathcal{O}_{\mathscr{A}_{\mathfrak{O} \mathfrak{p}}}\right) \rightarrow \mathcal{O}_{\mathfrak{p}} \llbracket t_{1, v}, \ldots, t_{g, v} \rrbracket .
$$

Celle-ci associe à l'image d'une section $f$ de $H^{0}\left(\mathscr{A}_{\mathscr{O}_{\mathfrak{p}}}, \mathcal{O}_{\mathscr{A}_{\mathfrak{O}_{\mathfrak{p}}}}\right)$ son développement de Taylor en 0 :

$$
\sum_{\mu \in \mathbb{N} g}\left(\partial_{v}^{\mu} f\right) \mathbf{t}_{v}^{\mu}
$$

On a, par la proposition 2.11 , les majorations suivantes :

$$
\forall 1 \leq i \leq g:\left|t_{i, v}\left(P_{k_{n}}\right)\right|_{\mathfrak{q}} \leq p^{-n_{\mathfrak{q}} / p} .
$$

De plus, pour tout $\mu \in \mathbb{N}^{g}$, la condition (2-3) garantit :

$$
\left|\partial_{v}^{\mu}\left(s \circ \tau_{x^{\prime}}\right)\right|_{\mathfrak{q}} \leq 1
$$

La norme étant ultramétrique, ceci suffit à voir que la série de Taylor de $\partial^{\lambda}\left(s \circ \tau_{x^{\prime}}\right)$ converge. On en déduit l'égalité :

$$
\partial_{x}^{\lambda} s=\sum_{\mu \in \mathbb{N} g} \partial_{\nu}^{\mu} \partial^{\lambda}\left(s \circ \tau_{x^{\prime}}\right) \mathbf{t}_{v}\left(P_{k_{n}}\right)^{\mu}
$$

Par définition de $E_{k}, s$ est nulle à un ordre $T_{n-1}$ en $x^{\prime}$, et cette propriété ne dépend pas du choix de la base de dérivations; donc si $\partial_{\nu}^{\mu} \partial^{\lambda}\left(s \circ \tau_{x^{\prime}}\right) \neq 0$, on a :

$$
\sum_{1 \leq i \leq g} \mu_{i}+\lambda_{i}>T_{n-1}
$$

On en déduit :

$$
\left|\prod_{i=1}^{g} t_{i, v}\left(P_{k_{n}}\right)^{\mu_{i}}\right|_{\mathfrak{q}} \leq p^{-n_{\mathfrak{q}}\left(T_{n-1}-T_{n}\right) / p} .
$$

La norme étant ultramétrique, l'inégalité fine de la proposition en résulte. 
5C. Normes archimédiennes des morphismes d'évaluation. Soit $k$ un entier, et $\sigma$ une place archimédienne de $K^{\prime}$. Tous les $\phi_{k}$ sont définis sur $K^{\prime}$. Le but de ce paragraphe est de majorer $\left\|\phi_{k}\right\|_{\sigma}$, par des méthodes d'analyse complexe, en particulier l'inégalité de Cauchy. Cette majoration sera la même pour toutes les places archimédiennes. On suit [Gaudron 2006, paragraphe 5.9].

Proposition 5.5. Il existe une constante $c_{15}$ ne dépendant que de A telle que:

$$
\frac{1}{\left[K^{\prime}: \mathbb{Q}\right]} \sum_{\sigma: K^{\prime} \hookrightarrow \mathbb{C}} \log \left\|\phi_{k}\right\|_{\sigma} \leq c_{15} T_{(k)} \log M .
$$

Démonstration. Soit $s \in E_{k} \otimes \mathbb{C}$ et $x \in \mathscr{X}_{k}$. On a alors :

$$
\phi_{k}(s)(x) \in\left(S^{T_{(k)}} t_{\mathscr{A}_{M}}^{\vee} \otimes x^{*} \mathscr{L}_{M}\right) \otimes_{\sigma} \mathbb{C},
$$

qui est isomorphe (non isométriquement) à :

$$
\operatorname{Hom}_{\mathbb{C}}\left(S^{T_{(k)}} t_{\mathscr{A}_{M, \sigma}}, x^{*} \mathscr{L}_{M, \sigma}\right) .
$$

Si on fixe une base d'ouverts affines, l'image de $\phi_{k}(s)(x)$ par cet isomorphisme est le morphisme qui associe à une dérivation $D$ d'ordre $T_{(k)}$ la valeur de $D s$ en $x$ (la dérivation $D$ donnant par translation une dérivation en $x$ ). On note $\Theta_{T_{(k)}}$ cet isomorphisme qui est défini dans [Gaudron 2006, 4.1], où sa norme d'opérateur, ainsi que celle de son inverse, sont majorées :

$$
\left\|\Theta_{T_{(k)}}\right\|_{\sigma} \leq \max _{\substack{\mathbf{i} \in \mathbb{N}^{g} \\|\mathbf{i}|=T_{(k)}}} \frac{T_{(k)} !}{\mathbf{i} !} \quad \text { et } \quad\left\|\Theta_{T_{(k)}}^{-1}\right\|_{\sigma} \leq 1 .
$$

On en déduit que :

$$
\left\|\phi_{k}(s)(x)\right\|_{\sigma} \leq\left\|\Theta_{T_{(k)}}\left(\phi_{k}(s)(x)\right)\right\|_{\sigma} .
$$

Soit $\left(f_{1, \sigma}, \ldots, f_{g, \sigma}\right)$ une base de $t_{A_{M, \sigma}}$ (composée à partir d'une base orthonormée de $t_{\mathscr{A}}$ pour la forme de Riemann induite par $\sigma$ ), correspondant à des dérivations $\left(\partial_{1, \sigma}, \ldots, \partial_{g, \sigma}\right)$. Soit $D$ une dérivation d'ordre $T_{(k)}$ le long de $t_{\unlhd_{M, \sigma}}$. On écrit

$$
D=\sum_{\substack{\mathbf{i} \in \mathbb{N} g \\|\mathbf{i}|=T_{(k)}}} d_{\mathbf{i}} \partial_{1, \sigma}^{i_{1}} \cdots \partial_{g, \sigma}^{i_{g}}
$$

La norme sur $S^{T_{(k)}} t_{\mathfrak{A}_{M, \sigma}}$ est la norme quotient déduite de la projection :

$$
t_{\mathfrak{A}_{M, \sigma}}^{\otimes T_{(k)}} \rightarrow S^{T_{(k)}} t_{\mathfrak{A}_{M, \sigma}}
$$

On a donc :

$$
\|D\|^{2}=\sum_{|\mathbf{i}|=T_{(k)}}\left|d_{\mathbf{i}}\right|^{2} \frac{\mathbf{i} !}{T_{(k)} !} \geq\left(\sum_{|\mathbf{i}|=T_{(k)}}\left|d_{\mathbf{i}}\right|\right)^{2} \times g^{-T_{(k)}}
$$


De plus, on a :

$$
\|D s(x)\| \leq \sum_{|\mathbf{i}|=T_{(k)}}\left|d_{\mathbf{i}}\right|\left\|\left(\partial_{1, \sigma}^{i_{1}} \cdots \partial_{g, \sigma}^{i_{g}}\right) s(x)\right\|,
$$

et on en déduit :

$$
\left\|\phi_{k}(s)(x)\right\|_{\sigma} \leq g^{T_{(k)} / 2} \max _{|\mathbf{i}|=T_{(k)}}\left\|\left(\partial_{1, \sigma}^{i_{1}} \cdots \partial_{g, \sigma}^{i_{g}}\right) s(x)\right\| .
$$

En reprenant la définition de la métrique cubiste, on peut se ramener à la fonction $\theta$ correspondant à $s$; notons que via le plongement étiré, la section $s$ est une section sur $A$ de degré $\leq 2 M^{2}$. On note $\mathbf{u}$ un logarithme de $\sigma(x)$ ayant une norme hermitienne minimale sur $\mathbb{C}^{g}$. En tenant compte de l'action de $\psi_{M}$ sur les dérivations, il vient :

$$
\left\|\phi_{k}(s)(x)\right\|_{\sigma} \leq(Q \sqrt{g})^{T_{(k)}} e^{-3 \pi M^{2}\|\mathbf{u}\|_{\sigma}^{2}} \max _{|\mathbf{i}|=T_{(k)}}\left|\frac{1}{\mathbf{i} !}\left(\frac{\partial}{\partial z}\right)^{\mathbf{i}} \theta(\mathbf{u}+\mathbf{z})_{\mid \mathbf{z}=0}\right| .
$$

De plus, par l'inégalité de Cauchy appliquée à $\theta$, pour tout réel $r_{C}>0$ (à ne pas confondre avec $r$, qui désigne la codimension de $V$ ), on a :

$$
\max _{|\mathbf{i}|=T_{(k)}}\left|\frac{1}{\mathbf{i} !}\left(\frac{\partial}{\partial z}\right)^{\mathbf{i}} \theta(\mathbf{u}+\mathbf{z})_{\mid \mathbf{z}=0}\right| \leq \frac{1}{r_{C}^{T_{(k)}}} \sup _{\|\mathbf{z}\|_{\sigma} \leq r_{C}}|\theta(\mathbf{u}+\mathbf{z})| .
$$

En revenant aux métriques cubistes, on trouve :

$$
\left\|\phi_{k}(s)(x)\right\|_{\sigma} \leq\left(\frac{M \sqrt{g}}{r_{C}}\right)^{T_{(k)}} e^{3 \pi M^{2}\left(r_{C}^{2}+2 r_{C}\|\mathbf{u}\|_{\sigma}\right)}\|s\|_{\text {sup }, \sigma} .
$$

On choisit alors $r_{C}$ de façon à optimiser la majoration :

$$
r_{C}=\frac{\sqrt{g}}{M^{2} \max \left\{1,\|\mathbf{u}\|_{\sigma}\right\}} .
$$

Comme $M^{2} \geq \sqrt{g}$ (par le choix des paramètres), cela donne :

$$
\left\|\phi_{k}(s)(x)\right\|_{\sigma} \leq\left(M^{3} \max \left\{1,\|\mathbf{u}\|_{\sigma}\right\}\right)^{T_{(k)}} e^{9 \pi g}\|s\|_{\text {sup }, \sigma} .
$$

La norme $\operatorname{sur} \mathscr{G}_{k}$ étant la norme de Löwner $\|\cdot\|_{L}$ associée à la norme du sup, elle est plus petite que celle-ci et on a finalement :

$$
\left\|\phi_{k}(s)\right\|_{L, \sigma} \leq\left(M^{3} \max \left\{1,\|\mathbf{u}\|_{\sigma}\right\}\right)^{T_{(k)}} e^{9 \pi g}\|s\|_{\text {sup }, \sigma},
$$

Et, vu le choix de la norme $L^{2}$ sur $\mathscr{E}_{M}$ :

$$
\left\|\phi_{k}\right\|_{\sigma} \leq\left(M^{3} \max \left\{1,\|\mathbf{u}\|_{\sigma}\right\}\right)^{T_{(k)}} e^{9 \pi g} \sup _{\substack{s \in \mathscr{E} M \\ s \neq 0}} \frac{\|s\|_{\text {sup }, \sigma}}{\|s\|_{\mathrm{L}^{2}, \sigma}} .
$$


La remarque 4.18 de [Gaudron 2006] montre que pour une certaine constante $c_{13}$ ne dépendant que de $g$ :

$$
\frac{1}{\left[K^{\prime}: \mathbb{Q}\right]} \sum_{\sigma: K^{\prime} \rightarrow \mathbb{C}} \log \max \left\{1,\|\mathbf{u}\|_{\sigma}\right\} \leq c_{13} \max \left\{1, \log ^{+} h_{F}(A), \log h^{0}(A, L)\right\} .
$$

De plus, d'après la même référence, lemme 4.16, il existe une autre constante $c_{14}$ telle que :

$$
\log \sup _{\substack{s \in \mathscr{C}, \mu \\ s \neq 0 \\ s \neq 0}} \frac{\|s\|_{\text {sup }, \sigma}}{\|s\|_{\mathrm{L}^{2}, \sigma}} \leq c_{14} \max \left\{1, \log ^{+}\left(h_{F}(A)\right), \log \left(h^{0}(A, L)\right)\right\} \log M^{2}
$$

La proposition suit en sommant sur les places archimédiennes, pour une constante $c_{15}$ dépendant de $A$.

Remarques. On aurait pu choisir différemment le paramètre $r_{C}$ issu de l'inégalité de Cauchy, en prenant à la place : $r_{C}^{\prime}=r_{C} \times M^{2}$. On aurait alors obtenu une majoration par $c_{15}\left(T_{(k)}+M^{2}\right)$, qui aurait été plus mauvaise dans notre contexte puisque tous les paramètres sauf $M$ sont logarithmiques en $\operatorname{deg}_{L}(X)$. Jusqu'à la phase de descente (où cela ne semble plus possible), on pourrait travailler avec des termes en $\log \omega_{L}(V)$ à la place de $\log \operatorname{deg}_{L} V$, quitte à améliorer la proposition 3.12.

5D. Inégalité de pentes et conséquences. Pour pouvoir appliquer le théorème des pentes, on doit s'assurer que le morphisme $\phi$ est injectif. On y travaillera dans la partie suivante, et on suppose par avance ici cette injectivité.

Contradiction sous l'injectivité de $\phi$. On suppose par l'absurde que (4-2) est vérifié. Puisqu'on a aussi supposé que $\phi$ est injectif, on peut donc utiliser l'inégalité des pentes du lemme 3.7 :

$$
\widehat{\operatorname{deg}} \overline{\mathscr{E}} M \leq \sum_{k \in I} \operatorname{dim}\left(E_{k} / E_{k+1}\right)\left(\hat{\mu}_{\max }\left(\overline{\mathscr{G}}_{k}\right)+h\left(\phi_{k}\right)\right) .
$$

Soit $k>0$ un entier. On veut d'abord montrer que la contribution du $k$-ème terme dans la somme précédente est négative. On regroupe pour commencer les estimations faites sur le morphisme $\phi_{k}$, à savoir les majorations archimédiennes et ultramétriques. L'entier $k$ est par définition $n$-lié à tout idéal premier $\mathfrak{q}$ de $0_{K^{\prime}}$ au-dessus d'un seul premier $\mathfrak{p}$ de $\mathscr{P}_{A}$. On note $p$ son image dans $\mathbb{Z}$; on a alors (par les propositions 5.4 et 5.5 ) :

$$
\begin{aligned}
h\left(\phi_{k}\right) & =\frac{1}{\left[K^{\prime}: \mathbb{Q}\right]} \sum_{v \in M\left(K^{\prime}\right)} \log \left\|\phi_{k}\right\|_{v} \leq c_{15} T_{n} \log M-\frac{1}{\left[K^{\prime}: \mathbb{Q}\right]} \sum_{\mathfrak{q} \mid \mathfrak{p}} n_{\mathfrak{q}}\left(T_{n-1}-T_{n}\right) \frac{\log p}{p} \\
& \leq c_{15} T_{n} \log M-\frac{1}{[K: \mathbb{Q}]}\left(T_{n-1}-T_{n}\right) \frac{\log p}{p},
\end{aligned}
$$


où on a utilisé l'égalité classique [Lang 1994, II, Corollary 1 to Theorem 2],

$$
\sum_{\mathfrak{q} \mid \mathfrak{p}} n_{\mathfrak{q}}=\left[K^{\prime}: K\right]
$$

On majore ensuite :

$$
\begin{aligned}
\hat{\mu}_{\max }\left(\overline{\bar{G}_{k}}\right)+h\left(\phi_{k}\right) \\
\quad \leq c_{16}\left(M^{2} \hat{\mu}_{L}^{\mathrm{ess}}(X)+T_{n} \log \left(M+T_{n}\right)+\log \operatorname{deg}_{L}(X)\right)-\frac{1}{[K: \mathbb{Q}]} T_{n-1} \frac{\log p}{p} \\
\quad \leq c_{16}\left(M^{2} \hat{\mu}_{L}^{\mathrm{ess}}(X)+T_{n} \log \left(M+T_{n}\right)+\log \operatorname{deg}_{L}(X)\right)-\frac{1}{[K: \mathbb{Q}]} T_{n-1} \frac{\log N_{n}}{N_{n}}
\end{aligned}
$$

pour une constante $c_{16}$ ne dépendant que de $A$, car $p \leq N_{n}$ et la fonction $\log (x) / x$ est décroissante pour $x \geq 3$. Le choix du paramètre $M$ (voir 4D) et l'hypothèse (4-2) sur le minimum essentiel donnent :

$$
M^{2} \hat{\mu}_{L}^{\mathrm{ess}}(X) \leq 2 \Delta .
$$

On a aussi, par l'hypothèse (4-1) et la définition des paramètres :

$$
\begin{aligned}
\log \left(M+T_{n}\right) & \leq \log \left(2 T_{0} \omega_{L}\left(\Delta T_{0}, X\right)\right) \leq \log \left(2 \Delta T_{0}^{2}\left|H_{0}\right| \operatorname{deg}_{L}(V)\right) \\
& \leq C_{0} \log (\Delta)+\Delta+\log \operatorname{deg}_{L}(V) \leq 2 \Delta .
\end{aligned}
$$

On en déduit :

$$
\hat{\mu}_{\max }\left(\bar{G}_{k}\right)+h\left(\phi_{k}\right) \leq c_{16}\left(2 \Delta+2 \Delta T_{n}+2 \Delta\right)-\frac{T_{n-1}}{N_{n}} \leq 6 c_{16} \Delta T_{n}-\frac{T_{n-1}}{N_{n}} \leq 0,
$$

la dernière inégalité résultant de la définition des $T_{n}$ par récurrence descendante. Il suit, puisque l'estimation qu'on vient de faire est encore valable pour $k=0$ sans le raffinement ultramétrique :

$$
\widehat{\operatorname{deg}} \overline{\mathscr{E}_{M}} \leq \operatorname{dim}\left(E_{0} / E_{1}\right)\left(\hat{\mu}_{\max }\left(\overline{\mathscr{G}_{0}}\right)+h\left(\phi_{0}\right)\right) \leq \operatorname{rg} \mathscr{G}_{0}\left(5 c_{16} \Delta T_{0}\right) .
$$

On a pu remplacer $\operatorname{dim}\left(E_{0} / E_{1}\right)$ par $\operatorname{rg}\left(\varphi_{0}\right)$ en utilisant l'injectivité de $\phi$, et parce que la hauteur est majorée par un terme positif. En combinant le lemme 4.2 (par le choix des paramètres que $\log M$ est plus grand que n'importe quelle constante) et le corollaire 5.3 avec $k=0$, on obtient :

$$
c_{10}(M)^{2 g} \log M \leq \frac{g\left(2 M^{2}\right)^{g}}{\Delta T_{0}}\left(5 c_{16} \Delta T_{0}\right),
$$

et on rappelle que $c_{10}>0$. On en déduit la contradiction suivante :

$$
\log M \leq \frac{5 g 2^{g} c_{16}}{c_{10}}
$$




\section{Lemme de zéros et injectivité du morphisme}

Il nous reste à assurer l'injectivité du morphisme de restriction. On procède par l'absurde, en commençant par écrire un lemme de zéros. Une étude combinatoire (paragraphe 6B) nous amène à une « quasi-contradiction » (paragraphe 6C). On conclut par un argument de descente sur des variétés (paragraphe 6D), qu'on détaille ici en petite codimension $r \leq 2$.

6A. Lemme de zéros. Le lemme de zéros dont on a besoin ici s'inscrit dans la tradition des théorèmes démontrés par Philippon [1995], dont on reprend le formalisme. Ce lemme est l'analogue abélien du théorème utilisé dans [Amoroso et David 2003], à une différence près : on prend en compte les multiplicités, et ce à l'aide de la notion de dessous d'escalier, qui permet d'envisager des multiplicités différenciées selon les directions. Dans le cas qui nous intéresse, il est utile d'envisager la multiplicité finale dans le lemme de zéros. On ne fait pas usage, cependant, de multiplicités différentes selon les directions.

Remarque. On a pris la multiplicité finale $T_{r}$ dans l'inégalité de pentes égale à 1 mais la multiplicité finale dans le lemme de zéros est un certain $T_{r_{0}}$, pour $r_{0} \leq r$, et n'est pas forcément nulle.

Soient $Z=\sum_{i} n_{i} \cdot Z_{i}$ et $Z^{\prime}=\sum_{i} n_{i}^{\prime} \cdot Z_{i}$ deux cycles algébriques (quitte à rajouter des zéros, on peut supposer que la somme porte sur les mêmes composantes). On définit leur réunion, notée $Z \cup Z^{\prime}$, comme le cycle $\sum \max _{i}\left\{n_{i}, n_{i}^{\prime}\right\} \cdot Z_{i}$. Le degré du cycle $\sum_{i} n_{i} \cdot Z_{i}$ sera l'entier $\sum_{i} n_{i} \operatorname{deg}_{L}\left(Z_{i}\right)$. Soit $A$ une variété abélienne munie d'un fibré $L$ ample. On considère la base de dérivations sur $A$ définie en $4 \mathrm{C}$. Un ensemble $E \subset \mathbb{N}^{g}$ est un escalier si pour tout $\beta \in E$, on a $\beta+\mathbb{N}^{g} \subset E$. Un sousensemble de $\mathbb{N}^{g}$ est un dessous d'escalier s'il est le complémentaire d'un escalier. Si $W$ est le dessous d'un escalier $E$ de $\mathbb{N}^{g}$, et si on a des indices $1 \leq i_{1}<\cdots<i_{r} \leq g$, on note $\mathscr{C}_{i_{1}, \ldots, i_{r}}(W)$ l'enveloppe convexe dans $\mathbb{R}_{+}^{r}$ de la trace de $E$ sur la $r$-face de $\mathbb{N}^{g}$ définie par $\left(i_{1}, \ldots, i_{r}\right)$.

On appelle aussi ensemble pondéré un sous-ensemble $\Sigma$ de $\mathbb{N}^{g} \times A$ tel que pour tout $x \in A$, l'ensemble $W_{x, \Sigma}=\left(\mathbb{N}^{g} \times\{x\}\right) \cap \Sigma$ soit un dessous d'escalier (éventuellement vide). On appelle support de $\Sigma$, noté $\operatorname{Supp}(\Sigma)$, sa projection sur $A$. Si $\Sigma$ et $\Sigma^{\prime}$ sont deux ensembles pondérés, on définit $\Sigma+\Sigma^{\prime}$ comme l'ensemble des couples $\left(x+x^{\prime}, \lambda+\lambda^{\prime}\right)$, pour $(x, \lambda) \in \Sigma$, et $\left(x^{\prime}, \lambda^{\prime}\right) \in \Sigma^{\prime}$; c'est aussi un ensemble pondéré. On a $E+\varnothing=\varnothing$ et si $E$ est un sous-ensemble de $A$, on l'identifie à l'ensemble pondéré $\{0\} \times E$.

On dit que $f \in H^{0}(A, L)$ s'annule sur un ensemble pondéré $\Sigma$ si pour tout $(x, \lambda) \in \Sigma$, on a $\partial_{x}^{\lambda} f=0$. Si $V$ est une sous-variété de codimension $r$ de $A$ et $W$ un dessous d'escalier, on pose :

$$
m_{W}(V)=r ! \max _{\substack{x \in V \\ 1 \leq i_{1}<\cdots<i_{r} \leq g}} \operatorname{vol}\left(\mathbb{R}_{+}^{r} \backslash \mathscr{C}_{i_{1}, \ldots, i_{r}}(W)\right) ;
$$


le maximum porte sur $x \in X$ et les $r$-faces de $\mathbb{N}^{g}$ telles que $\left(\partial_{i_{1}, x}, \ldots, \partial_{i_{r}, x}\right)$ forment une base du quotient $t_{A, x} / t_{V, x}$.

Théorème 6.1. Soit $V$ une sous-variété de A, de codimension $r, \tilde{M} \geq 1$ un entier et $\Sigma_{0}, \ldots, \Sigma_{r}$ des ensembles pondérés finis à support dans $A(\bar{K})$ tels que pour tout $1 \leq n \leq r$ :

$$
\operatorname{Supp}\left(\Sigma_{n}\right)=\bigcup_{l=1 \ldots s_{n}} H_{n, l},
$$

où les $H_{n, l}$ sont des sous-groupes de $A(\bar{K})$; et pour tout $x \in \operatorname{Supp}\left(\Sigma_{n}\right), W_{x, \Sigma_{n}}$ ne dépend pas de $x$. Soit de plus $f \in H^{0}\left(A, L^{\otimes \tilde{M}}\right)$, non nulle, qui s'annule sur $V+\Sigma_{0}+\cdots+\Sigma_{r}$. Alors il existe une constante $c_{17}$ ne dépendant que de A, deux entiers $1 \leq r_{0} \leq r_{1} \leq r$, des indices $j_{0}, \ldots, j_{r_{0}-1}$ avec $1 \leq j_{l} \leq s_{l}$ pour $l=0 \ldots r_{0}-1$, et des sous-variétés algébriques $Z_{j}\left(j=1, \ldots, s_{r_{0}}\right)$ strictes de A, de codimension $r_{1}$, contenant au moins une composante isolée de

$$
\operatorname{Supp}\left(H_{0, j_{0}}+\cdots+H_{r_{0}-1, j_{r_{0}-1}}+\Sigma_{r_{0}}+\cdots+\Sigma_{r}+V\right),
$$

telles que:

$$
\operatorname{deg}_{L}\left(\bigcup_{x \in H_{0, j_{0}}+\cdots+H_{r_{0}-1, j_{r_{0}-1}}} \bigcup_{\substack{j=1 \ldots s_{r_{0}} \\ y \in H_{r_{0}, j}}} m_{W_{y, \Sigma_{r_{0}}}}\left(x+y+Z_{j}\right) \cdot\left(x+y+Z_{j}\right)\right) \leq c_{17} \tilde{M}^{r_{1}} .
$$

Démonstration. Il s'agit d'un cas particulier du résultat principal de [Galateau 2009], énoncé dans le cadre plus général des groupes algébriques commutatifs.

6B. Degré d'une sous-variété obstructrice. On reprend les hypothèses et notations des parties 4 et 5 et on rappelle que :

$$
X=V+H_{0}
$$

où $V$ est une variété et $H_{0}$ est un sous-groupe fini de $A$. On suppose enfin que le cardinal $\left|H_{0}\right|$ est premier à tous les premiers des $\mathscr{P}_{n, \mathbb{Z}}$, pour $1 \leq n \leq r$. Si $p$ est un nombre premier de $\bigcup_{1 \leq n \leq r} \mathscr{P}_{n, \mathbb{Z}}$ et $\mathfrak{p} \mid p$ dans $\mathscr{O}_{K}$, on désigne par $\operatorname{Ker}[p]_{\mathfrak{p}}$ le groupe des points de $p$-torsion se réduisant sur 0 modulo $\mathfrak{p}$.

Si $l=\prod_{n=1}^{r} p_{n}$ avec $p_{n} \in \mathscr{P}_{n, \mathbb{Z}} \cup\{1\}$ pour tout $1 \leq n \leq r$, on note :

$$
\operatorname{Ker}[l]^{*}=\bigoplus_{n} \operatorname{Ker}\left[p_{n}\right]_{\mathfrak{p}_{n}} .
$$

Cette somme est bien directe car le choix des paramètres implique que les $\mathscr{P}_{n, \mathbb{Z}}$ sont deux-à-deux disjoints. Notre but, jusqu'à la fin de cette partie, sera de démontrer la proposition suivante, pour un bon choix du fermé $X$ (dont dépend la construction du morphisme) :

Proposition 6.2. Le morphisme $\phi: E \rightarrow F$ est injectif. 
On va supposer que ce n'est pas le cas et obtenir une contradiction en appliquant le lemme de zéros du paragraphe précédent. Celui-ci permet de majorer le degré d'une réunion de sous-variétés. On souhaite se ramener à une seule sous-variété obstructrice, et utiliser le fait que la réunion est largement distincte. On y arrive par un travail sur le stabilisateur, dont on rappelle la définition :

Définition 6.3. Si $Z$ est un fermé inclus dans $A$, on appelle stabilisateur de $Z$, noté $\operatorname{Stab}(Z)$, le groupe :

$$
\{x \in A: x+Z=Z\}=\bigcap_{x \in Z}(Z-x) .
$$

On a les propriétés suivantes (voir [Amoroso et David 1999, 2], qui se transpose immédiatement aux variétés abéliennes) :

$$
\operatorname{dim} \operatorname{Stab}(Z) \leq \operatorname{dim} Z, \quad \operatorname{deg}_{L}(\operatorname{Stab}(Z)) \leq \operatorname{deg}_{L}(Z)^{\operatorname{dim} Z+1} .
$$

Proposition 6.4. Il existe une constante $c_{19}$, des entiers $r_{0} \leq r_{1} \leq r$ strictement positifs, un entier $l \in \mathscr{P}_{1, \mathbb{Z}} \cdots \mathscr{P}_{r_{0}, \mathbb{Z}}$, et une sous-variété $Z$ stricte de A, de codimension $r_{1}$ contenant un translaté de $V$ par un point de torsion, tels que:

$$
T_{r_{0}}^{r_{1}}\left|\mathscr{P}_{r, \mathbb{Z}}\right| \frac{\left|H_{0}\right|}{\left|H_{0} \cap \operatorname{Stab}(Z)\right|} \frac{l^{g}}{\left|\operatorname{Ker}[l]^{*} \cap \operatorname{Stab}(Z)\right|} \operatorname{deg}_{L}(Z) \leq c_{19} M^{2 r_{1}} \Delta .
$$

Pour simplifier les calculs qui viennent, on pose :

$$
f\left(H_{0}, Z\right)=\frac{\left|H_{0}\right|}{\left|\operatorname{Stab}(Z) \cap H_{0}\right|} .
$$

Démonstration. Si le morphisme $\phi$ n'est pas injectif, il existe une section $f \in$ $H^{0}\left(A, L^{\otimes M^{2}+1}\right)$ qui s'annule sur $\bigcup_{i \in I} V\left(X_{i}, t_{A}, T_{(i)}\right)$. Par définition des voisinages infinitésimaux, ceci implique que $f$ s'annule sur $X+\Sigma_{1}+\cdots+\Sigma_{r}$, où l'ensemble $\Sigma_{n}$, pour $1 \leq n \leq r$, est pondéré de support :

$$
\operatorname{Supp}\left(\Sigma_{n}\right)=\operatorname{Tor}_{A, n}=\bigcup_{\mathfrak{p} \in \mathscr{P}_{n}} \operatorname{Ker}[p]_{\mathfrak{p}}
$$

et de dessous d'escalier ne dépendant pas de $x \in \operatorname{Supp}\left(\Sigma_{n}\right)$ défini par :

$$
\sum_{k=1}^{g} \lambda_{k} \leq T_{n} .
$$

Il existe donc, par le théorème précédent, deux entiers $r_{0}$ et $r_{1}$ tels que $r_{0} \leq r_{1} \leq r$, des couples d'idéaux premiers $\left(p_{1}, \mathfrak{p}_{1}\right), \ldots,\left(p_{r_{0}-1}, \mathfrak{p}_{r_{0}-1}\right)$ avec $\mathfrak{p}_{n} \in \mathscr{P}_{n}$ (pour $1 \leq$ $n \leq r_{0}-1$ ) et des sous-variétés strictes $Z_{\mathfrak{p}}$ de $A$ (pour tout $\mathfrak{p} \in \mathscr{P}_{r_{0}}$ ), de codimension 
$r_{1}$, tels que :

$$
\operatorname{deg}_{L}\left(\bigcup_{\mathfrak{p} \in \mathscr{P}_{r_{0}}} \bigcup_{\zeta \in \bigoplus_{n} \operatorname{Ker}\left[p_{n}\right]_{\mathfrak{p}_{n}} \oplus H_{0}} m_{W_{\zeta r_{0}, \Sigma r_{0}}}\left(\zeta+Z_{\mathfrak{p}}\right) \cdot\left(\zeta+Z_{\mathfrak{p}}\right)\right) \leq 2^{r_{1}} c_{17} M^{2 r_{1}},
$$

où on a écrit $\mathfrak{p}_{r_{0}}=\mathfrak{p}$, pour unifier l'écriture dans la somme directe, et où $\zeta_{r_{0}}$ est la composante selon $r_{0}$ de $\zeta$ dans la somme directe. De plus, pour tout $\mathfrak{p} \in \mathscr{P}_{r_{0}}$, la variété $Z_{\mathfrak{p}}$ contient un translaté de $V$ par un point de torsion.

La multiplicité se calcule immédiatement. Soit $\mathfrak{p} \in \mathscr{P}_{r_{0}}$ et

$$
\zeta \in H_{0} \oplus \bigoplus_{n} \operatorname{Ker}\left[p_{n}\right]_{\mathfrak{p}_{n}}
$$

on a :

$$
m_{W_{\zeta r_{0}, \Sigma_{r_{0}}}}\left(\zeta+Z_{\mathfrak{p}}\right)=r_{1} ! T_{r_{0}}^{r_{1}} \operatorname{vol}\left(\left\{u_{1}+\cdots+u_{r_{1}}<1\right\}\right)=T_{r_{0}}^{r_{1}} .
$$

Cette multiplicité ne dépend pas de la variété dans la réunion, donc on peut la mettre en facteur.

Les entiers $r_{0}$ et $r_{1}$ sont déjà déterminés; posons $l_{0}=p_{1} \cdots p_{r_{0}-1}$. Choisissons, pour tout premier $p \in \mathscr{P}_{r_{0}, \mathbb{Z}}$, un idéal premier $\mathfrak{p}$ de $\mathscr{P}_{r_{0}}$ divisant $p$ tel que la quantité :

$$
\frac{f\left(H_{0}, Z_{\mathfrak{p}}\right)\left(l_{0} p\right)^{g}}{\left|\operatorname{Ker}\left[l_{0} p\right]^{*} \cap \operatorname{Stab}\left(Z_{\mathfrak{p}}\right)\right|} \operatorname{deg}_{L}\left(Z_{\mathfrak{p}}\right)
$$

soit minimale parmi les premiers de $\mathscr{P}_{r_{0}}$ divisant $p$. Prenons aussi $\mathfrak{p}_{r_{0}} \in \mathscr{P}_{r_{0}}$ (et $p_{r_{0}}$ ) tels que cette même quantité soit minimale parmi tous les premiers de $\mathscr{P}_{r_{0}}$. On pose $l=l_{0} p_{r_{0}}$ et $Z=Z_{\mathfrak{p}_{r_{0}}}$. Il suffit donc de majorer cette quantité pour obtenir la proposition. Rappelons que la somme :

$$
\operatorname{Ker}\left[l_{0}\right]^{*}=\bigoplus_{n=1}^{r_{0}-1} \operatorname{Ker}\left[p_{n}\right]_{\mathfrak{p}_{n}}
$$

est bien directe car, les premiers $p_{n}$ étant deux-à-deux distincts, on peut écrire une relation de Bézout entre un des $p_{n}$ et tous les autres. On partitionne $\mathscr{P}_{r_{0}, \mathbb{Z}}$ en introduisant la relation d'équivalence suivante :

$p \sim p^{\prime} \Longleftrightarrow$ il existe $\gamma \in H_{0} \oplus \operatorname{Ker}\left[l_{0}\right]^{*} \oplus \bigoplus_{p_{i} \in \mathscr{P}_{r_{0}, \mathbb{Z}}} \operatorname{Ker}\left[p_{i}\right]_{\mathfrak{p}_{i}}$ tel que $\gamma+Z_{\mathfrak{p}}=Z_{\mathfrak{p}^{\prime}}$, et on note $\left(\mathscr{C}_{1}, \ldots, \mathscr{C}_{s}\right)$ les différentes classes d'équivalence associées. Si $p$ et $p^{\prime}$ appartiennent à des classes différentes, les réunions

$$
\bigcup_{\zeta \in H_{0} \oplus \operatorname{Ker}\left[l_{0}\right]^{*} \oplus \operatorname{Ker}[p]_{\mathfrak{p}}} \zeta+Z_{\mathfrak{p}}
$$

n'ont aucune composante en commun et on peut additionner les degrés. Le choix d'un seul idéal de $\mathscr{P}_{r_{0}}$ au-dessus d'un nombre premier restreint la réunion. On a 
donc :

$$
T_{r_{0}}^{r_{1}} \sum_{j=1}^{s} \operatorname{deg}_{L}\left(\bigcup_{p \in \mathscr{C}_{j}} \bigcup_{\zeta \in H_{0} \oplus \operatorname{Ker}\left[l_{0}\right]^{*} \oplus \operatorname{Ker}[p]_{\mathfrak{p}}} \zeta+Z_{\mathfrak{p}}\right) \leq 2^{r_{1}} c_{17} M^{2 r_{1}}
$$

Soit $p \in \mathscr{P}_{r_{0}, \mathbb{Z}}$ et soit $\mathfrak{p}$ l'idéal qui lui est associé ; le stabilisateur de $Z_{\mathfrak{p}}$ ne dépend que de la classe d'équivalence de $p$ puisque si $p^{\prime}$ (associé à $\mathfrak{p}^{\prime}$ ) est dans la même classe que $p, Z_{\mathfrak{p}^{\prime}}$ est un translaté de $Z_{\mathfrak{p}}$. On appelle $\mathscr{S}_{j}$ le stabilisateur commun aux $Z_{\mathfrak{p}}$, pour $\mathfrak{p}$ associé à $p \in \mathscr{C}_{j}$. Dans chaque classe $\mathscr{C}_{j}$, on fixe un premier $\rho_{j} \in \mathscr{C}_{j}$ et on note $Z_{\rho_{j}}$ la variété qui lui est associée. Pour tout autre premier $p \in \mathscr{C}_{j}$, il existe donc des éléments $\alpha_{p} \in \bigoplus_{p_{i} \in \mathscr{P}_{r_{0}, \mathbb{Z}}} \operatorname{Ker}\left[p_{i}\right]_{\mathfrak{p}_{i}}$ et $\eta_{p} \in H_{0} \oplus \operatorname{Ker}\left[l_{0}\right]^{*}$ tels que :

$$
\alpha_{p}+\eta_{p}+Z_{\mathfrak{p}}=Z_{\rho_{j}}
$$

Remarquons que la somme est directe car tous les $p_{i}$ sont distincts. Soient $p \neq p^{\prime}$ dans la même classe $\mathscr{C}_{j}$; soient $\omega_{p} \in \operatorname{Ker}[p]_{\mathfrak{p}}$ et $\omega_{p^{\prime}} \in \operatorname{Ker}\left[p^{\prime}\right]_{\mathfrak{p}^{\prime}}$. Si les réunions

$$
\bigcup_{\zeta \in H_{0} \oplus \operatorname{Ker}\left[l_{0}\right]^{*}} \zeta+\omega_{\xi}+Z_{\xi}
$$

pour $\xi=p$ et $\xi=p^{\prime}$ ont au moins une composante commune, c'est qu'il existe un élément $\eta_{p, p^{\prime}} \in H_{0} \oplus \operatorname{Ker}\left[l_{0}\right]^{*}$ tel que :

$$
\omega_{p}+Z_{p}=\eta_{p, p^{\prime}}+\omega_{p^{\prime}}+Z_{p^{\prime}}
$$

On en déduit, grâce aux deux dernières égalités, que :

$$
x=\alpha_{p}-\omega_{p}-\alpha_{p^{\prime}}+\omega_{p^{\prime}}+\left(\eta_{p}-\eta_{p^{\prime}}+\eta_{p, p^{\prime}}\right) \in \mathscr{S}_{j}
$$

On note $\alpha_{p}^{p_{i}}$ la composante selon $p_{i}$ de $\alpha_{p}$. On remarque que : $\alpha_{p}^{p}-\alpha_{p^{\prime}}^{p}-\omega_{p} \in$ $\operatorname{Ker}[p]_{\mathfrak{p}}$. De même, $\alpha_{p^{\prime}}^{p^{\prime}}-\alpha_{p}^{p^{\prime}}-\omega_{p^{\prime}} \in \operatorname{Ker}\left[p^{\prime}\right]_{\mathfrak{p}^{\prime}}$ et $\eta_{p^{\prime}}-\eta_{p}+\eta_{p, p^{\prime}} \in H_{0} \oplus \operatorname{Ker}\left[l_{0}\right]^{*}$.

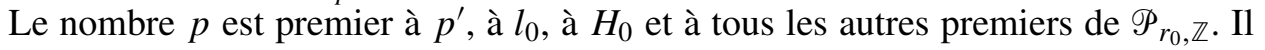
existe donc une relation de Bézout :

$$
u p+v l_{0} \prod_{p_{i} \neq p \in \mathscr{P}_{r_{0}, \mathbb{Z}}} p_{i}=1
$$

On en déduit que :

$$
\left[v l_{0} \prod_{p_{i} \neq p \in \mathscr{P}_{r_{0}, \mathbb{Z}}} p_{i}\right] x=\left[v l_{0} \prod_{p_{i} \neq p \in \mathscr{P}_{r_{0}, \mathbb{Z}}} p_{i}\right]\left(\alpha_{p}^{p}-\alpha_{p^{\prime}}^{p}-\omega_{p}\right)=\alpha_{p}^{p}-\alpha_{p^{\prime}}^{p}-\omega_{p} \in \mathscr{S}_{j}
$$

puisqu'il suit de sa définition que le stabilisateur est stable sous la multiplication par $n$, quel que soit $n \in \mathbb{N}$. Par contraposition, si :

$$
\omega_{p} \in \operatorname{Ker}[p]_{\mathfrak{p}} \backslash\left(\alpha_{p}^{p}-\alpha_{p^{\prime}}^{p}-\mathscr{Y}_{j}\right) \quad \text { et } \quad \omega_{p^{\prime}} \in \operatorname{Ker}\left[p^{\prime}\right]_{\mathfrak{p}^{\prime}} \backslash\left(\alpha_{p^{\prime}}^{p^{\prime}}-\alpha_{p}^{p^{\prime}}+\mathscr{Y}_{j}\right)
$$


les réunions (6-1) n'ont pas de composantes communes. Il suit :

$$
\begin{aligned}
\operatorname{deg}_{L}\left(\bigcup_{p \in \mathscr{C}_{j}} \bigcup_{\zeta \in H_{0} \oplus \operatorname{Ker}\left[l_{0}\right]^{*}} \bigcup_{\xi \in \operatorname{Ker}[p]_{\mathfrak{p}}} \zeta+\xi+Z_{\mathfrak{p}}\right) \\
\quad \geq \sum_{p \in \mathscr{C}_{j}} \operatorname{deg}_{L}\left(\bigcup_{\zeta \in H_{0} \oplus \operatorname{Ker}\left[l_{0}\right]^{*}} \bigcup_{\xi \in \operatorname{Ker}[p]_{\mathfrak{p}} \backslash \bigcup_{i}\left(\alpha_{p}^{p}-\alpha_{p_{i}}^{p}+\mathscr{\varphi}_{j}\right)} \zeta+\xi+Z_{\mathfrak{p}}\right) .
\end{aligned}
$$

Fixons $j$ et $p \in \mathscr{C}_{j}$. On va calculer le degré de la réunion totale en fonction de $\operatorname{deg}_{L}\left(Z_{\mathfrak{p}}\right)$. Par choix de l'ensemble $\mathscr{P}_{A}$, il y a $p^{g}$ points se réduisant sur $0 \bmod \mathfrak{p}$, et il y a $l_{0}^{g}$ points dans $\operatorname{Ker}\left[l_{0}\right]^{*}$. Il en résulte :

$$
\operatorname{deg}_{L}\left(\bigcup_{\zeta \in H_{0} \oplus \operatorname{Ker}\left[l_{0}\right]^{*}} \bigcup_{\xi \in \operatorname{Ker}[p]_{\mathfrak{p}}} \zeta+\xi+Z_{\mathfrak{p}}\right)=\frac{f\left(H_{0}, Z_{\mathfrak{p}}\right)\left(l_{0} p\right)^{g}}{\left|\mathcal{S}_{j} \cap \operatorname{Ker}\left[l_{0} p\right]^{*}\right|} \operatorname{deg}_{L}\left(Z_{\mathfrak{p}}\right)
$$

A cette réunion, il faut retrancher :

$$
\operatorname{deg}_{L}\left(\bigcup_{\zeta \in H_{0} \oplus \operatorname{Ker}\left[l_{0}\right]^{*}} \bigcup_{\xi \in \bigcup_{i}\left(\alpha_{p}^{p}-\alpha_{p_{i}}^{p}+\mathscr{S}_{j}\right)} \zeta+\xi+Z_{\mathfrak{p}}\right) \leq \frac{f\left(H_{0}, Z_{\mathfrak{p}}\right)\left|\mathscr{C}_{j}\right| l_{0}^{g}}{\left|\mathscr{S}_{j} \cap \operatorname{Ker}\left[l_{0}\right]^{*}\right|} \operatorname{deg}_{L}\left(Z_{\mathfrak{p}}\right) .
$$

En effet, il y a au plus $\left|\mathscr{C}_{j}\right|$ points de la forme $\alpha_{p_{i}}^{p}$. Notons $\tilde{\mathscr{C}}_{j}$ le sous-ensemble de $\mathscr{C}_{j}$ formé des $p$ divisant $\left[\mathscr{Y}_{j}: \mathscr{S}_{j}^{0}\right]$, où $\mathscr{S}_{j}^{0}$ désigne la composante connexe de l'identité dans $\mathscr{S}_{j}$. Si $p \notin \tilde{\mathscr{C}}_{j}$, les dénominateurs des deux dernières formules sont égaux et on a :

$$
\begin{array}{r}
\operatorname{deg}_{L}\left(\bigcup_{\zeta \in H_{0} \oplus \operatorname{Ker}\left[l_{0}\right]^{*} \xi \in \operatorname{Ker}[p]_{\mathfrak{p}} \backslash \bigcup_{i}\left(\alpha_{p}^{p}-\alpha_{p_{i}}^{p}+\mathscr{S}_{j}\right)} \bigcup+\xi+Z_{\mathfrak{p}}\right) \\
\geq\left(1-\frac{\left|\mathscr{C}_{j}\right|}{p^{2}}\right) \frac{f\left(H_{0}, Z_{\mathfrak{p}}\right)\left(l_{0} p\right)^{g}}{\left|\mathscr{S}_{j} \cap \operatorname{Ker}\left[l_{0} p\right]^{*}\right|} \operatorname{deg}_{L}\left(Z_{\mathfrak{p}}\right) .
\end{array}
$$

Le quotient $1 / p^{2}$ provient du fait que la $\ll$ partie discrète $\gg$ du stabilisateur de $Z_{\mathfrak{p}}$ est triviale et que sa composante connexe en 0 est un groupe algébrique de codimension $\geq r+1 \geq 2$. En fixant $j$, on somme sur l'ensemble des $p$; en tenant compte de la définition de $Z$, on obtient :

$$
\begin{aligned}
\operatorname{deg}_{L}\left(\bigcup_{p \in \mathscr{C}_{j}} \bigcup_{\zeta \in H_{0} \oplus \operatorname{Ker}\left[l_{0}\right]^{*}} \bigcup_{\xi \in \operatorname{Ker}[p]_{\mathfrak{p}}} \zeta+Z_{\mathfrak{p}}\right) \\
\geq\left(\left|\mathscr{C}_{j} \backslash \tilde{\mathscr{C}}_{j}\right|-\left|\mathscr{C}_{j}\right| \sum_{p \in \mathscr{C}_{j}} \frac{1}{p^{2}}\right) \frac{f\left(H_{0}, Z\right)(l)^{g}}{\left|\operatorname{Ker}[l]^{*} \cap \operatorname{Stab}(Z)\right|} \operatorname{deg}_{L}(Z) \\
\geq\left(\frac{2}{3}\left|\mathscr{C}_{j}\right|-\left|\tilde{\mathscr{C}}_{j}\right|\right) \frac{f\left(H_{0}, Z\right)(l)^{g}}{\left|\operatorname{Ker}[l]^{*} \cap \operatorname{Stab}(Z)\right|} \operatorname{deg}_{L}(Z),
\end{aligned}
$$


par la définition de l'ensemble $\mathscr{P}_{A}$. On a plus directement, par (6-2) et la définition de $Z$ :

$$
\operatorname{deg}_{L}\left(\bigcup_{p \in \mathscr{C}_{j}} \bigcup_{\zeta \in H_{0} \oplus \operatorname{Ker}\left[l_{0}\right]^{*}} \bigcup_{\xi \in \operatorname{Ker}[p]_{\mathfrak{p}}} \zeta+Z_{\mathfrak{p}}\right) \geq \frac{f\left(H_{0}, Z\right)(l)^{g}}{\left|\operatorname{Ker}[l]^{*} \cap \operatorname{Stab}(Z)\right|} \operatorname{deg}_{L}(Z)
$$

On doit donc estimer le nombre de premiers divisant $\left[\mathscr{S}_{j}: \mathscr{S}_{j}^{0}\right]$. Or :

$$
\left|\tilde{\mathscr{C}}_{j}\right| \leq \frac{\log \left[\mathscr{Y}_{j}: \mathscr{Y}_{j}^{0}\right]}{\log 3} \leq \log \operatorname{deg}_{L}\left(\mathscr{Y}_{j}\right) \leq c_{18} \Delta,
$$

pour une constante $c_{18}$. On a ici majoré le degré du stabilisateur en fonction de celui de la variété [Hindry 1988, lemme 6], puis on a utilisé le lemme de zéros pour majorer $\operatorname{deg}_{L}\left(Z_{\rho_{j}}\right)$, et on a majoré $\log M$ à l'aide du choix des paramètres. Par l'inégalité $\max \{x-y ; 1\} \geq x / 2 y$ pour $x \geq 0$ et $y \geq 1$, on obtient :

$$
\max \left\{\frac{2}{3}\left|\mathscr{C}_{j}\right|-\left|\tilde{\mathscr{C}}_{j}\right|, 1\right\} \geq \frac{\left|\mathscr{C}_{j}\right|}{3 c_{18} \Delta} \text {. }
$$

La proposition suit en sommant sur les classes d'équivalence.

6C. Un premier pas vers l'injectivité. Le choix des paramètres va donner une inégalité «presque absurde"; on ne pourra cependant pas conclure, car il manquera une hypothèse de coprimalité sur des objets construits simultanément pendant la phase diophantienne.

Proposition 6.5. On suppose que $X$ n'est pas incluse dans le translaté d'une sousvariété abélienne et que son minimum essentiel est majoré de la façon suivante:

$$
\hat{\mu}_{L}^{\mathrm{ess}}(X) \omega_{L}(X)<\frac{1}{\Delta^{8 \rho(2 r)^{r+1}}} .
$$

Alors il existe une sous-variété stricte $Z$ de codimension $r_{1} \leq r$ contenant un translaté de $V$ par un point de torsion et un entier $l>0$ tels que:

- L'entier l est premier avec $R$ et:

$$
l \leq \Delta^{2 \rho(2 r)^{r+1}} .
$$

- De plus, on a l'inégalité:

$$
\left(\frac{f\left(H_{0}, Z\right) l^{g}}{\left|\operatorname{Ker}[l]^{*} \cap \operatorname{Stab}(Z)\right|} \operatorname{deg}_{L}(Z)\right)^{1 / r_{1}}<\Delta^{-\rho} l \omega_{L}(l, X) .
$$

Démonstration. On commence par montrer que la contrainte portant ici sur le minimum essentiel est plus forte que l'hypothèse (4-2). Si (4-2) n'est pas vérifiée :

$$
\hat{\mu}_{L}^{\mathrm{ess}}(X) \geq \frac{\Delta}{T_{0} \omega_{L}\left(\Delta T_{0}, X\right)} \geq \frac{1}{T_{0}^{2} \omega_{L}(X)},
$$


par application du lemme 4.5. De plus, par les choix de paramètres faits en 4D :

$$
T_{0}^{2} \leq \Delta^{2 r} \prod_{n=1}^{r} N_{n}^{4} \leq \Delta^{8 \rho(2 r)^{r+1}},
$$

ce qui contredit l'hypothèse de la proposition. Pour démontrer cette inégalité, on a d'abord utilisé :

$$
N_{1} \cdots N_{r} \leq \Delta^{\rho\left[(2 r)^{2}+\cdots+(2 r)^{r+1}\right]}
$$

puis on a majoré l'exposant comme suit :

$$
\sum_{j=2}^{r+1}(2 r)^{j} \leq-r+\sum_{j=1}^{r+1}(2 r)^{j} \leq 2(2 r)^{r+1}-r
$$

par l'inégalité :

$$
1+x+\cdots+x^{h} \leq 2 x^{h} \quad \text { pour } h \in \mathbb{N} \text { et } x \geq 2 .
$$

La proposition précédente nous donne donc l'existence de trois entiers strictement positifs $r_{0}, r_{1}, l$ avec $r_{0} \leq r_{1} \leq r$ et $l \in \mathscr{P}_{1, \mathbb{Z}} \cdots \mathscr{P}_{r_{0}, \mathbb{Z}}$, et une sous-variété algébrique $Z$ stricte de $A$, de codimension $r_{1}$, contenant un translaté de $V$ par un point de torsion, telle que :

$$
T_{r_{0}}^{r_{1}}\left|\mathscr{P}_{r_{0}, \mathbb{Z}}\right| \frac{f\left(H_{0}, Z\right) l^{g}}{\left|\operatorname{Ker}[l]^{*} \cap \operatorname{Stab}(Z)\right|} \operatorname{deg}_{L}(Z) \leq c_{19} M^{2 r_{1}} \Delta .
$$

Par construction des $\mathscr{P}_{n, \mathbb{Z}}$, l'entier $l$ est premier avec $R$ et on a les inégalités :

$$
2^{-r_{0}} N_{1} \cdots N_{r_{0}} \leq l \leq N_{1} \cdots N_{r_{0}} .
$$

Et le premier point suit, par la même majoration que (6-3).

Reste à prouver la seconde inégalité. Le théorème des nombres premiers et le choix de l'ensemble $\mathscr{P}_{A}$ font que, pour une certaine constante $c_{20}>0$ ne dépendant que de $A$ :

$$
\left|\mathscr{P}_{r_{0}, \mathbb{Z}}\right| \geq c_{20} \frac{N_{r_{0}}}{\log N_{r_{0}}}-\frac{\log R}{\log 2} .
$$

Par définition des $N_{n}$ et de $\Delta$, on a $\log N_{r_{0}} \leq \Delta^{1 / 2}$ pour $C_{0}$ assez grand dans la définition de $\Delta$. On a aussi :

$$
N_{r_{0}} \geq \Delta^{9} \geq \log (R)^{2} .
$$

On a encore, pour $C_{0}$ assez grand, $\frac{1}{2} c_{20} \Delta^{1 / 2} \geq 1$, le facteur $\frac{1}{2}$ correspondant au terme en $\log R$. On en déduit :

$$
\left|\mathscr{P}_{r_{0}, \mathbb{Z}}\right| \geq \Delta^{\rho(2 r)^{r+2-r_{0}-1}} .
$$

Par [Amoroso et David 2003, lemme 2.4], comme $l \leq N_{1} \cdots N_{r} \leq \Delta T_{0}$, on a :

$$
\omega_{L}\left(\Delta T_{0}, X\right) \leq \frac{\Delta T_{0}}{l} \omega_{L}(l, X) .
$$


Puis :

$$
\begin{aligned}
& \left(\frac{f\left(H_{0}, Z\right) l^{g}}{\left|\operatorname{Ker}[l]^{*} \cap \operatorname{Stab}(Z)\right|} \operatorname{deg}_{L}(Z)\right)^{1 / r_{1}} \leq \frac{c_{19} M^{2} \Delta^{1 / r_{1}}}{\left|\mathscr{P}_{r_{0}, \mathbb{Z}}\right|^{1 / r_{1}} T_{r_{0}}} \\
& \leq 2 c_{19} \frac{T_{0} \omega_{L}\left(\Delta T_{0}, X\right) \Delta^{1 / r_{1}}}{\left|\mathscr{P}_{r_{0}, \mathbb{Z}}\right|^{1 / r_{1}} T_{r_{0}}} \\
& \leq 2 c_{19} \frac{\Delta T_{0}^{2} \Delta^{1 / r_{1}}}{l\left|\mathscr{P}_{r_{0}, \mathbb{Z}}\right|^{1 / r_{1}} T_{r_{0}}} \omega_{L}(l, X) \text {. }
\end{aligned}
$$

Or on a :

$$
\frac{T_{0}}{T_{r_{0}}} \leq \Delta^{2 r_{0}} N_{1} \cdots N_{r_{0}} \leq(2 \Delta)^{2 r_{0}} l
$$

et on en déduit :

$$
\left(\frac{f\left(H_{0}, Z\right) l^{g}}{\left|\operatorname{Ker}[l]^{*} \cap \operatorname{Stab}(Z)\right|} \operatorname{deg}_{L}(Z)\right)^{1 / r_{1}} \leq c_{21} \frac{l \Delta^{2 r+1+1 / r_{1}} N_{r_{0}+1} \cdots N_{r}}{\left|\mathscr{P}_{r_{0}, \mathbb{Z}}\right|^{1 / r_{1}}} \omega_{L}(l, X) .
$$

L'exposant $h$ de $\Delta$ dans cette dernière majoration est borné par :

$$
\begin{aligned}
h & :=4 r+2 \rho\left((2 r)^{2}+\cdots+(2 r)^{r+1-r_{0}}\right)-\left(\rho(2 r)^{r+2-r_{0}}-2\right) / r_{1} . \\
& \leq 2 \rho\left((2 r)+\cdots+(2 r)^{r+1-r_{0}}\right)-2 \rho(2 r)^{r+1-r_{0}} \\
& \leq 2 \rho\left(r-r_{0}\right)(2 r)^{r-r_{0}}+\rho(2 r)^{r+1-r_{0}}-2 \rho(2 r)^{r+1-r_{0}} \\
& \leq-2 \rho r_{0}(2 r)^{r-r_{0}} \leq-2 \rho .
\end{aligned}
$$

On a donc finalement :

$$
\left(\frac{f\left(H_{0}, Z\right) l^{g}}{\left|\operatorname{Ker}[l]^{*} \cap \operatorname{Stab}(Z)\right|} \operatorname{deg}_{L}(Z)\right)^{1 / r_{1}}<\Delta^{-\rho} l \omega_{L}(l, X),
$$

en faisant disparaître les constantes avec $\Delta^{\rho}$, et le résultat suit.

On notera dorénavant :

$$
\left|\operatorname{Ker}[l]^{*} \cap \operatorname{Stab}(Z)\right|=\lambda(Z, l) .
$$

Remarque. Posons $X=V$ et $H_{0}=\{0\}$. Si on savait assurer la coprimalité entre $l$ et $\left[\operatorname{Stab}(Z): \operatorname{Stab}(Z)^{0}\right]$, on pourrait déjà clore la preuve, car on aurait :

$$
\lambda(Z, l) \leq l^{\operatorname{dim} \operatorname{Stab}(Z)^{0}} \leq l^{\left(g-r_{1}-1\right)},
$$

la deuxième inégalité provenant du fait que $V$ n'est pas inclus dans un translaté de sous-variété abélienne. La variété $Z$ contenant un translaté de $V$ par un point de torsion, on a de plus :

$$
\omega_{L}(l, V) \leq\left(l \operatorname{deg}_{L}(Z)\right)^{1 / r_{1}}
$$

et une contradiction suivrait immédiatement. 
6D. Itération et descente. Pour conclure, on est donc amené à itérer la dernière proposition, suivant une stratégie de descente désormais classique dans les minorations de hauteur par voie diophantienne. On détaille ici la descente en codimension $r \leq 2$, où la technicité est moins grande et ne cache pas les idées combinatoires en jeu.

Démonstration du théorème 1.8 en codimension $r \leq 2$. Soit $V$ une sous-variété stricte de $A$ qui n'est pas incluse dans un translaté de sous-variété abélienne de $A$, de codimension $r \leq 2$. On rappelle que :

$$
\Delta=C_{0}^{2} \log \left(3 \operatorname{deg}_{L}(V)\right),
$$

et on suppose :

$$
\omega_{L}(V) \hat{\mu}_{L}^{\mathrm{ess}}(V)<\Delta^{-\left(16(2 r)^{r+1}\right)^{r}} .
$$

Première étape. Pour utiliser la proposition 6.5, on doit définir :

$$
\rho_{1}=\left(9(2 r)^{r+1}\right)^{r-1} \quad \text { et } \quad R_{1}=\left[\operatorname{Stab}(V): \operatorname{Stab}(V)^{0}\right] .
$$

On a, en tenant compte des propriétés du stabilisateur suivant la définition6.3 :

$$
\log R_{1} \leq \log \operatorname{deg}_{L}(\operatorname{Stab}(V)) \leq g \log \left(3 \operatorname{deg}_{L}(V)\right) \leq \Delta .
$$

Si le morphisme $\phi$ n'est pas injectif, on applique la proposition 6.5 avec $X=V$, ce qui donne l'existence d'un entier $l_{1}$ et d'une sous-variété $Z_{1}$ de $A$, stricte et de codimension $k_{1}$, contenant un translaté de $V$ par un point $x_{1}$, et telle que :

$$
\left(\frac{l_{1}^{g} \operatorname{deg}_{L}\left(Z_{1}\right)}{\lambda\left(Z_{1}, l_{1}\right)}\right)^{1 / k_{1}}<\Delta^{-\rho_{1}} l_{1} \omega_{L}\left(l_{1}, V\right) .
$$

On peut supposer que $V$ est de codimension 2 et que $Z_{1}$ est une hypersurface. Sinon, on aurait $Z_{1}=x_{1}+V$, l'entier $l_{1}$ serait premier à

$$
\left[\operatorname{Stab}(V): \operatorname{Stab}(V)^{0}\right]=\left[\operatorname{Stab}\left(Z_{1}\right): \operatorname{Stab}\left(Z_{1}\right)^{0}\right],
$$

et la remarque suivant la preuve de la proposition 6.5 montre qu'on aurait une contradiction.

Deuxième étape. On itère maintenant la proposition 6.5 en posant :

$$
\begin{gathered}
V_{1}=\bigcup_{x \in \operatorname{Stab}\left(Z_{1}\right) \cap \operatorname{Ker}\left[l_{1}\right]^{*}} x+V, \quad \rho_{2}=\left(9(2 r)^{r+1}\right)^{r-2}, \\
R_{2}=\left[\operatorname{Stab}(V): \operatorname{Stab}(V)^{0}\right] \times\left[\operatorname{Stab}\left(Z_{1}\right): \operatorname{Stab}\left(Z_{1}\right)^{0}\right] \times l_{1} .
\end{gathered}
$$

La dernière condition permet que le cardinal de $H_{0}$ soit premier à tous les premiers des $\mathscr{P}_{i, \mathbb{Z}}$ dans la phase combinatoire. On vérifie une nouvelle fois (par les majorations du degré de $Z_{1}$ et de $l_{1}$ données par la proposition 6.5) que :

$$
\log R_{2} \leq g \log \left(3 \omega_{L}(V)\right)+g^{2} \log \omega_{L}(V)+3 \log l_{1} \leq \Delta .
$$


L'hypothèse (4-1) est satisfaite pour les mêmes raisons (on a $\left|H_{0}\right| \leq l_{1}^{2 g}$ ). On doit aussi majorer $\omega_{L}\left(V_{1}\right) \hat{\mu}_{L}^{\text {ess }}\left(V_{1}\right)$. Le minimum essentiel de $V_{1}$ est celui de $V$. Comme $x_{1}+V_{1} \subset Z_{1}$ (par définition de ces deux variétés), l'inégalité sur le degré de $Z_{1}$ donne :

$$
\omega_{L}\left(V_{1}\right) \leq l_{1} \omega_{L}\left(l_{1}, V\right) \leq l_{1}^{2} \omega_{L}(V)
$$

On obtient donc :

$$
\begin{aligned}
\omega_{L}\left(V_{1}\right) \hat{\mu}_{L}^{\mathrm{ess}}\left(V_{1}\right) & \leq l_{1}^{4} \omega_{L}(V) \hat{\mu}_{L}^{\mathrm{ess}}(V) \leq \Delta^{-\left(16(2 r)^{r+1}\right)^{r}+8 \rho_{1}(2 r)^{r+1}} \\
& \leq \Delta^{\rho_{2}(2 r)^{(r+1)}(-16+8)} \leq \Delta^{-8 \rho_{2}(2 r)^{r+1}}
\end{aligned}
$$

Par (6-5), on a enfin :

$$
C_{0} \log \left(3 \omega_{L}\left(V_{1}\right)\right) \leq \Delta .
$$

La proposition 6.5 avec $H_{0}=\operatorname{Stab}\left(Z_{1}\right) \cap \operatorname{Ker}\left[l_{1}\right]^{*}$ donne l'existence d'une variété $Z_{2}$ de codimension $k_{2}$ contenant un translaté $x_{2}+V$, telle que :

$$
\left(\frac{f\left(H_{0}, Z_{2}\right) l_{2}^{g} \operatorname{deg}_{L}\left(Z_{2}\right)}{\lambda\left(Z_{2}, l_{2}\right)}\right)^{1 / k_{2}}<\Delta^{-\rho_{2}} l_{2} \omega_{L}\left(l_{2}, V_{1}\right) .
$$

Or, $Z_{2}$ contient les translatés de $x_{2}+V$ par les points de $H_{0} \cap \operatorname{Stab}\left(Z_{2}\right)$. On a :

$$
\operatorname{deg}_{L}\left(\bigcup_{x \in H_{0} /\left(H_{0} \cap \operatorname{Stab} Z_{2}\right)} x+Z_{2}\right) \leq f\left(H_{0}, Z_{2}\right) \operatorname{deg}_{L}\left(Z_{2}\right) \text {; }
$$

et cette réunion, notée $Z_{2}^{\prime}$, contient un translaté de $V_{1}$. Si $Z_{2}$ est de codimension 2, on a $Z_{2}=x_{2}+V$, et on en déduit que $l_{2}$ est premier à $\left[\operatorname{Stab}\left(Z_{2}\right): \operatorname{Stab}\left(Z_{2}\right)^{0}\right]$. Il suit :

$$
\left(l_{2} \operatorname{deg}_{L}\left(Z_{2}^{\prime}\right)\right)^{1 / 2} \leq \Delta^{-\rho_{2}} \omega_{L}\left(l_{2}, V_{1}\right),
$$

ce qui est absurde, puisque $Z_{2}^{\prime}$ contient un translaté de $V_{1}$.

Les deux variétés $Z_{1}$ et $Z_{2}$ sont donc des hypersurfaces, qui contiennent toutes deux un translaté de $V$, de codimension 2. Quitte à translater ces deux variétés (ce qui est sans conséquences sur le degré et le stabilisateur), on suppose que $V \subset$ $Z_{1} \cap Z_{2}$. Il reste à comparer $Z_{1}$ et $Z_{2}$ pour finir la preuve.

Cas 1. L'intersection $Z_{1} \cap Z_{2}$ est de codimension 1 . Les deux hypersurfaces (irréductibles) sont donc égales. Par construction, $Z_{1}$ contient $V_{1}$ et on a :

$$
\omega_{L}\left(V_{1}\right) \leq \operatorname{deg}_{L}\left(Z_{1}\right) \leq \operatorname{deg}_{L}\left(Z_{2}\right) .
$$

En outre, l'égalité des variétés nous montre que $l_{2}$ est premier à la partie discrète du stabilisateur de $Z_{2}$, et comme cette hypersurface n'est pas incluse dans un translaté de variété abélienne (puisque cette propriété est vraie pour $V \subset Z_{2}$ ) :

$$
\omega_{L}\left(V_{1}\right) \leq \Delta^{-\rho_{2}} l_{2}^{(2-g)} \lambda\left(Z_{2}, l_{2}\right) \omega_{L}\left(V_{1}\right) \leq \Delta^{-\rho_{2}} \omega_{L}\left(V_{1}\right) .
$$

On obtient donc une contradiction. 
Cas 2. L'intersection $Z_{1} \cap Z_{2}$ est de codimension 2. Dans ce cas, cette intersection contient $V$, mais elle contient aussi les translatés de $V$ par les points de $H_{0} \cap$ $\operatorname{Stab}\left(Z_{2}\right)$. Comme ce groupe est de cardinal une puissance de $l_{1}$, la partie discrète du stabilisateur de $V$ n'intervient pas et on a :

$\operatorname{deg}_{L}\left(\bigcup_{x \in H_{0} \cap \operatorname{Stab}\left(Z_{2}\right)} x+V\right) \geq \frac{\left|H_{0} \cap \operatorname{Stab}\left(Z_{2}\right)\right|}{l_{1}^{(\operatorname{dim} V-1)}} \operatorname{deg}_{L}(V)=\frac{\lambda\left(Z_{1}, l_{1}\right) l_{1}^{(3-g)}}{f\left(H_{0}, Z_{2}\right)} \operatorname{deg}_{L}(V)$.

On a utilisé au passage le fait que $V$ n'était pas un translaté de variété abélienne. Par le théorème de Bézout, il vient :

$$
\begin{aligned}
\frac{\lambda\left(Z_{1}, l_{1}\right) l_{1}^{(3-g)}}{f\left(H_{0}, Z_{2}\right)} \operatorname{deg}_{L}(V) & \leq \operatorname{deg}_{L}\left(Z_{1}\right) \operatorname{deg}_{L}\left(Z_{2}\right) \\
& \leq \Delta^{-\rho_{1}} \frac{\lambda\left(Z_{1}, l_{1}\right) l_{1}^{(1-g)}}{f\left(H_{0}, Z_{2}\right)} l_{2}^{4} \omega_{L}\left(l_{1}, V\right) \omega_{L}\left(V_{1}\right) .
\end{aligned}
$$

La majoration des termes en $l_{2}$ a été grossière car ceux-ci sont négligeables devant $\Delta^{\rho_{1}}$ par la majoration de $l_{2}$ suivant la proposition 6.5. On en déduit :

$$
\operatorname{deg}_{L}(V) \leq \Delta^{-\rho_{1}} l_{1}^{-2} l_{2}^{4} \omega_{L}\left(l_{1}, V\right) \omega_{L}\left(V_{1}\right) .
$$

Par (6-5), on trouve :

$$
l_{1} \operatorname{deg}_{L}(V) \leq \Delta^{-\rho_{1}} l_{2}^{4} \omega_{L}\left(l_{1}, V\right)^{2} \leq \Delta^{u} \omega_{L}\left(l_{1}, V\right)^{2},
$$

et le réel $u$ vérifie :

$$
u \leq-\rho_{1}+8 \rho_{2}(2 r)^{r+1}<0 .
$$

C'est à nouveau une contradiction.

Fin de la preuve. On a donc démontré par l'absurde que la proposition 6.2 était vraie, soit avec $X=V$, soit avec $X=V_{1}$. Il en résulte dans les deux cas que $V$ contredit la majoration (6-4). On en déduit :

$$
\hat{\mu}_{L}^{\mathrm{ess}}(V) \geq \frac{C_{L}(A)}{\omega_{L}(V)}\left(\log \left(3 \operatorname{deg}_{L}(V)\right)\right)^{-\lambda(r)},
$$

où $\lambda(r)=\left(16(2 r)^{(r+1)}\right)^{r}$ et $C_{L}(A)=1 / C_{0}^{2 \lambda(r)}$, qui ne dépend que de $A$.

Conclusion. Expliquons pour finir quelles modifications il convient d'apporter à ce travail pour traiter la descente en codimension générale, suivant [Galateau 2008]. La principale difficulté rencontrée dans le cas abélien est l'absence, en général, d'un relèvement du morphisme de Frobenius en caractéristique nulle. On est donc amené à le reconstituer combinatoirement, en considérant le translaté d'une variété $V$ par certains sous-groupes de torsion. La phase de descente, lorsqu'on travaille avec des fermés réductibles, est particulièrement délicate et nécessite la définition de nouveaux indices d'obstructions. 
Pour préparer au mieux cette descente, on rajoute une information d'ordre combinatoire dans la phase de transcendance. Plus précisément, en insérant un principe de tiroirs de Dirichlet dans le lemme de zéros, on montre que le fermé $Z$ donné par la proposition 6.5 peut être choisi de telle sorte qu'il contienne une proportion « raisonnable $\gg$ de composantes irréductibles de $X$; on perd en contrepartie l'irréductibilité de $Z$. À quelques détails près dans le choix des paramètres, on obtient alors [Galateau 2008, proposition 5.1], sur laquelle s'articule la descente finale.

\section{Remerciements}

Je souhaite remercier chaleureusement Sinnou David, qui m'a patiemment initié au problème de Bogomolov au cours de mon doctorat, et Eric Gaudron pour sa minutieuse relecture et ses conseils. Pendant la rédaction de ce travail, j'ai pu bénéficier des explications d'Antoine Chambert-Loir, Huayi Chen, Richard Pink, Hugues Randriam et Emmanuel Ullmo. Je remercie enfin le rapporteur dont les commentaires m'ont permis d'améliorer significativement cet article.

\section{References}

[Amoroso et David 1999] F. Amoroso et S. David, "Le problème de Lehmer en dimension supérieure”, J. Reine Angew. Math. 513 (1999), 145-179. MR 2001a:11116 Zbl 1011.11045

[Amoroso et David 2003] F. Amoroso et S. David, "Minoration de la hauteur normalisée dans un tore”, J. Inst. Math. Jussieu 2:3 (2003), 335-381. MR 2004m:11101 Zbl 1041.11048

[Bombieri et Zannier 1995] E. Bombieri et U. Zannier, "Algebraic points on subvarieties of $\mathbf{G}_{m}^{n}$ ", Internat. Math. Res. Notices 7 (1995), 333-347. MR 96h:11061 Zbl 0848.11030

[Bombieri et Zannier 1996] E. Bombieri et U. Zannier, "Heights of algebraic points on subvarieties of abelian varieties", Ann. Scuola Norm. Sup. Pisa Cl. Sci. (4) 23:4 (1996), 779-792. MR 98j:11043 Zbl 0897.11020

[Bombieri et al. 1999] E. Bombieri, D. Masser et U. Zannier, "Intersecting a curve with algebraic subgroups of multiplicative groups", Int. Math. Res. Not. 1999:20 (1999), 1119-1140. MR 2001c: 11081 Zbl 0938.11031

[Bombieri et al. 2007] E. Bombieri, D. Masser et U. Zannier, "Anomalous subvarieties - structure theorems and applications", Int. Math. Res. Not. 2007:19 (2007), ID rnm057. MR 2008k:11060 Zbl 1145.11049

[Bost 1996a] J.-B. Bost, "Intrinsic heights of stable varieties and abelian varieties", Duke Math. J. 82:1 (1996), 21-70. MR 97j:14025 Zbl 0867.14010

[Bost 1996b] J.-B. Bost, "Périodes et isogenies des variétés abéliennes sur les corps de nombres (d'après D. Masser et G. Wüstholz)”, pp. 115-161 dans Séminaire Bourbaki, 1994/95 (exposé 795), Astérisque 237, 1996. MR 98k:11073 Zbl 0936.11042

[Bost 2001] J.-B. Bost, "Algebraic leaves of algebraic foliations over number fields", Publ. Math. Inst. Hautes Études Sci. 93 (2001), 161-221. MR 2002h:14037 Zbl 1034.14010 
[Bost et Künnemann 2007] J. B. Bost et K. Künnemann, "Hermitian vector bundles and extension groups on arithmetic varieties, I: Geometry of numbers", prépubl., 2007. arXiv math.NT/0701343

[Bost et al. 1994] J.-B. Bost, H. Gillet et C. Soulé, "Heights of projective varieties and positive Green forms”, J. Amer. Math. Soc. 7:4 (1994), 903-1027. MR 95j:14025 Zbl 0973.14013

[Carrizosa 2009] M. Carrizosa, "Petits points et multiplication complexe", Int. Math. Res. Not. 2009:16 (2009), 3016-3097. MR 2533796 Zbl 1176.11025

[Chardin 1989] M. Chardin, "Une majoration de la fonction de Hilbert et ses conséquences pour l'interpolation algébrique”, Bull. Soc. Math. France 117:3 (1989), 305-318. MR 90m:13021 Zbl 0709.13007

[Chardin 1990] M. Chardin, Contributions à l'algèbre commutative effective et à la théorie de l'élimination, Thèse de Doctorat, Université Paris VI, 1990.

[Chen 2006] H. Chen, Positivité en géométrie algébrique et en géométrie d'Arakelov, Thèse de Doctorat, Université Paris XI, 2006.

[David 1991] S. David, "Fonctions thêta et points de torsion des variétés abéliennes", Compositio Math. 78:2 (1991), 121-160. MR 92d:11061 Zbl 0741.14025

[David et Hindry 2000] S. David et M. Hindry, "Minoration de la hauteur de Néron-Tate sur les variétés abéliennes de type C.M.”, J. Reine Angew. Math. 529 (2000), 1-74. MR 2001j:11054 Zbl 0993.11034

[David et Philippon 2000] S. David et P. Philippon, "Sous-variétés de torsion des variétés semiabéliennes", C. R. Acad. Sci. Paris Sér. I Math. 331:8 (2000), 587-592. MR 2001k:14084 Zbl 0972.11059

[David et Philippon 2002] S. David et P. Philippon, "Minorations des hauteurs normalisées des sousvariétés de variétés abeliennes, II”, Comment. Math. Helv. 77:4 (2002), 639-700. MR 2004a:11055 Zbl 1030.11026

[David et Philippon 2007] S. David et P. Philippon, "Minorations des hauteurs normalisées des sous-variétés des puissances des courbes elliptiques", Int. Math. Res. Pap. 3 (2007), ID rpm006. MR 2008h:11068 Zbl 1163.11049

[Demazure et Grothendieck 1970] M. Demazure et A. Grothendieck (éditeurs), Schémas en groupes, I: Propriétés générales des schémas en groupes, Lecture Notes in Mathematics 151, Springer, Berlin, 1970. MR 43 \#223a

[Galateau 2007] A. Galateau, Problème de Bogomolov sur les variétés abéliennes, Thèse de Doctorat, Université Paris VI, 2007.

[Galateau 2008] A. Galateau, "Une minoration du minimum essentiel sur les variétés abéliennes", prépublication, 2008. À paraître dans Comment. Math. Helv. arXiv 0807.0171

[Galateau 2009] A. Galateau, "Un théorème de zéros dans les groupes algébriques commutatifs. D’après Amoroso et David”, prépublication, 2009, Voir http://www.math.unibas.ch/ galateau/ zeros.pdf.

[Gaudron 2006] É. Gaudron, "Formes linéaires de logarithmes effectives sur les variétés abéliennes”, Ann. Sci. École Norm. Sup. (4) 39:5 (2006), 699-773. MR 2008a:11084 Zbl 1111.11038

[Gaudron 2008] É. Gaudron, "Pentes des fibrés vectoriels adéliques sur un corps global”, Rend. Semin. Mat. Univ. Padova 119 (2008), 21-95. MR 2009j:11107 Zbl 05582423

[Graftieaux 2001] P. Graftieaux, "Formal groups and the isogeny theorem", Duke Math. J. 106:1 (2001), 81-121. MR 2002f:14055 Zbl 1064.14045 
[Griffiths et Harris 1978] P. Griffiths et J. Harris, Principles of algebraic geometry, Wiley, New York, 1978. MR 80b:14001 Zbl 0408.14001

[Habegger 2009] P. Habegger, "A Bogomolov property for curves modulo algebraic subgroups", Bull. Soc. Math. France 137:1 (2009), 93-125. MR 2010d:11071 Zbl 05558321

[Hindry 1988] M. Hindry, “Autour d'une conjecture de Serge Lang”, Invent. Math. 94:3 (1988), 575-603. MR 89k:11046 Zbl 0638.14026

[Hindry et Silverman 2000] M. Hindry et J. H. Silverman, Diophantine geometry, Graduate Texts in Mathematics 201, Springer, New York, 2000. MR 2001e:11058 Zbl 0948.11023

[Ireland et Rosen 1990] K. Ireland et M. Rosen, A classical introduction to modern number theory, 2ème éd., Graduate Texts in Math. 84, Springer, New York, 1990. MR 92e:11001 Zbl 0712.11001

[Koblitz 1975] N. Koblitz, " $p$-adic variation of the zeta-function over families of varieties defined over finite fields”, Compositio Math. 31:2 (1975), 119-218. MR 54 \#2658 Zbl 0332.14008

[Lang 1994] S. Lang, Algebraic number theory, 2ème éd., Graduate Texts in Mathematics 110, Springer, New York, 1994. MR 95f:11085 Zbl 0811.11001

[Lang 2002] S. Lang, Algebra, 3rd éd., Graduate Texts in Mathematics 211, Springer, New York, 2002. MR 2003e:00003 Zbl 0984.00001

[Lange et Ruppert 1985] H. Lange et W. Ruppert, "Complete systems of addition laws on abelian varieties”, Invent. Math. 79:3 (1985), 603-610. MR 86f:14029 Zbl 0577.14035

[Laurent 1983] M. Laurent, "Minoration de la hauteur de Néron-Tate", pp. 137-151 dans Seminar on number theory (Paris, 1981/1982), édité par M.-J. Bertin, Progr. Math. 38, Birkhäuser, Boston, 1983. MR 85e:11048 Zbl 0521.14010

[Maurin 2008] G. Maurin, "Courbes algébriques et équations multiplicatives", Math. Ann. 341:4 (2008), 789-824. MR 2009g:14026 Zbl 1154.14017

[Moret-Bailly 1990] L. Moret-Bailly, "Sur l'équation fonctionnelle de la fonction thêta de Riemann”, Compositio Math. 75:2 (1990), 203-217. MR 92a:14049 Zbl 0728.14039

[Mumford 1974] D. Mumford, , 2éme éd., Tata Institute of Fundamental Research Studies in Mathematics 5, Oxford University Press, London, 1974. MR 2010e:14040 Zbl 0326.14012

[Noot 1995] R. Noot, "Abelian varieties - Galois representation and properties of ordinary reduction”, Compositio Math. 97:1-2 (1995), 161-171. MR 97a:11093 Zbl 0868.14021

[Ogus 1982] A. Ogus, Hodge cycles and crystalline cohomology, Lecture Notes in Mathematics 900, Springer, Berlin, 1982. MR 84m:14046 Zbl 0538.14010

[Philippon 1995] P. Philippon, "Sur des hauteurs alternatives, III", J. Math. Pures Appl. (9) 74:4 (1995), 345-365. MR 97a:11098 Zbl 0878.11025

[Pink 1998] R. Pink, " $l$-adic algebraic monodromy groups, cocharacters, and the Mumford-Tate conjecture”, J. Reine Angew. Math. 495 (1998), 187-237. MR 98m:11060 Zbl 0920.14006

[Pink 2004] R. Pink, "Finite group schemes", notes de cours, 2004, Voir http://www.math.ethz.ch/ $\sim$ pink/FiniteGroupSchemes.html.

[Pink 2005] R. Pink, "A common generalization of the conjectures of André-Oort, Manin-Mumford and Mordell-Lang”, prépublication, 2005, Voir www.math.ethz.ch/ pink/ftp/AOMMML.pdf.

[Ratazzi 2008] N. Ratazzi, "Intersection de courbes et de sous-groupes et problèmes de minoration de dernière hauteur dans les variétés abéliennes C.M.", Ann. Inst. Fourier (Grenoble) 58:5 (2008), 1575-1633. MR 2009m:11096 Zbl 1156.11025 
[Raynaud 1974] M. Raynaud, "Schémas en groupes de type $(p, \ldots, p)$ ", Bull. Soc. Math. France 102 (1974), 241-280. MR 54 \#7488 Zbl 0325.14020

[Rémond et Viada 2003] G. Rémond et E. Viada, "Problème de Mordell-Lang modulo certaines sous-variétés abéliennes”, Int. Math. Res. Not. 2003:35 (2003), 1915-1931. MR 2004h:11054 Zbl 1072.11038

[Samuel 1967] P. Samuel, Théorie algébrique des nombres, Hermann, Paris, 1967. MR 35 \#6643 Zbl 0146.06402

[Serre 1968] J.-P. Serre, Abelian l-adic representations and elliptic curves, Benjamin, New York, 1968. MR 41 \#8422 Zbl 0186.25701

[Viada 2003] E. Viada, "The intersection of a curve with algebraic subgroups in a product of elliptic curves", Ann. Sc. Norm. Super. Pisa Cl. Sci. (5) 2:1 (2003), 47-75. MR 2004c:11099 Zbl .11314 Zbl 1170.11314

[Viada 2008] E. Viada, "The intersection of a curve with a union of translated codimension-two subgroups in a power of an elliptic curve", Algebra Number Theory 2:3 (2008), 249-298. MR 2009f:11079 Zbl 1168.11024

[Viada 2009] E. Viada, "Nondense subsets of varieties in a power of an elliptic curve", Int. Math. Res. Not. 2009:7 (2009), 1213-1246. MR 2010d:14031 Zbl 1168.14030

[Zhang 1992] S. Zhang, "Positive line bundles on arithmetic surfaces", Ann. of Math. (2) 136:3 (1992), 569-587. MR 93j:14024 Zbl 0788.14017

[Zhang 1995a] S. Zhang, "Positive line bundles on arithmetic varieties", J. Amer. Math. Soc. 8:1 (1995), 187-221. MR 95c:14020 Zbl 0861.14018

[Zhang 1995b] S. Zhang, "Small points and adelic metrics", J. Algebraic Geom. 4:2 (1995), 281300. MR 96e:14025 Zbl 0861.14019

[Zilber 2002] B. Zilber, "Exponential sums equations and the Schanuel conjecture", J. London Math. Soc. (2) 65:1 (2002), 27-44. MR 2002m:11104 Zbl 1030.11073

Communicated by Bjorn Poonen

Received 2009-06-25 Revised 2009-11-16 Accepted 2009-12-20

aurelien.galateau@math.u-psud.fr

Mathematisches Institut, Universität Basel, Rheinsprung, 21, $\mathrm{CH}-4051$ Basel, Switzerland

Bâtiment 425, Université Paris-Sud, 91405 Orsay, France http://www.math.u-psud.fr/ galateau 


\section{Algebra \& Number Theory}

www.jant.org

\section{EDITORS}

\section{MANAGING EDITOR}

Bjorn Poonen

Massachusetts Institute of Technology

Cambridge, USA

\author{
EDITORIAL BOARD CHAIR \\ David Eisenbud \\ University of California \\ Berkeley, USA
}

\section{BOARD OF EDITORS}

Georgia Benkart

Dave Benson

Richard E. Borcherds

John H. Coates

J-L. Colliot-Thélène

Brian D. Conrad

Hélène Esnault

Hubert Flenner

Edward Frenkel

Andrew Granville

Joseph Gubeladze

Ehud Hrushovski

Craig Huneke

Mikhail Kapranov

Yujiro Kawamata

János Kollár

Hendrik W. Lenstra

Yuri Manin

Barry Mazur
University of Wisconsin, Madison, USA

University of Aberdeen, Scotland

University of California, Berkeley, USA

University of Cambridge, UK

CNRS, Université Paris-Sud, France

University of Michigan, USA

Universität Duisburg-Essen, Germany

Ruhr-Universität, Germany

University of California, Berkeley, USA

Université de Montréal, Canada

San Francisco State University, USA

Hebrew University, Israel

University of Kansas, USA

Yale University, USA

University of Tokyo, Japan

Princeton University, USA

Universiteit Leiden, The Netherlands

Northwestern University, USA

Harvard University, USA
Susan Montgomery

Shigefumi Mori

Andrei Okounkov

Raman Parimala

Victor Reiner

Karl Rubin

Peter Sarnak

Michael Singer

Ronald Solomon

Vasudevan Srinivas

J. Toby Stafford

Bernd Sturmfels

Richard Taylor

Ravi Vakil

Michel van den Bergh

Marie-France Vignéras

Kei-Ichi Watanabe

Andrei Zelevinsky

Efim Zelmanov
University of Southern California, USA

RIMS, Kyoto University, Japan

Princeton University, USA

Emory University, USA

University of Minnesota, USA

University of California, Irvine, USA

Princeton University, USA

North Carolina State University, USA

Ohio State University, USA

Tata Inst. of Fund. Research, India

University of Michigan, USA

University of California, Berkeley, USA

Harvard University, USA

Stanford University, USA

Hasselt University, Belgium

Université Paris VII, France

Nihon University, Japan

Northeastern University, USA

University of California, San Diego, USA

\section{PRODUCTION}

ant@mathscipub.org

Silvio Levy, Scientific Editor

Andrew Levy, Production Editor

See inside back cover or www.jant.org for submission instructions.

The subscription price for 2010 is US \$140/year for the electronic version, and \$200/year (+\$30 shipping outside the US) for print and electronic. Subscriptions, requests for back issues from the last three years and changes of subscribers address should be sent to Mathematical Sciences Publishers, Department of Mathematics, University of California, Berkeley, CA 94720-3840, USA.

Algebra \& Number Theory (ISSN 1937-0652) at Mathematical Sciences Publishers, Department of Mathematics, University of California, Berkeley, CA 94720-3840 is published continuously online. Periodical rate postage paid at Berkeley, CA 94704, and additional mailing offices.

ANT peer review and production are managed by EditFLOW ${ }^{\mathrm{TM}}$ from Mathematical Sciences Publishers.

PUBLISHED BY

mathematical sciences publishers

http://www.mathscipub.org

A NON-PROFIT CORPORATION

Typeset in LATEX

Copyright $\odot 2010$ by Mathematical Sciences Publishers 


\section{Algebra \& Number Theory}

Volume $4 \quad$ No. $5 \quad 2010$

On the Spiegelungssatz for the 4-rank

ÉTIENNE FOUVRY and JÜRGEN KLÜNERS

The Manin constant of elliptic curves over function fields AMBRUS PÁL

Le problème de Bogomolov effectif sur les variétés abéliennes

AURÉLIEN GALATEAU

Transverse quiver Grassmannians and bases in affine cluster algebras GRÉGOIRE DUPONT

Connected gradings and the fundamental group 